University of Rhode Island

DigitalCommons@URI

Open Access Dissertations

2017

\title{
Global Dynamics and Boundedness of Discrete Population Models
}

David T. McArdle

University of Rhode Island, DMcArdle@uri.edu

Follow this and additional works at: https://digitalcommons.uri.edu/oa_diss

\section{Recommended Citation}

McArdle, David T., "Global Dynamics and Boundedness of Discrete Population Models" (2017). Open Access Dissertations. Paper 593.

https://digitalcommons.uri.edu/oa_diss/593

This Dissertation is brought to you for free and open access by DigitalCommons@URI. It has been accepted for inclusion in Open Access Dissertations by an authorized administrator of DigitalCommons@URI. For more information, please contact digitalcommons-group@uri.edu. 
GLOBAL DYNAMICS AND BOUNDEDNESS OF DISCRETE POPULATION MODELS

BY

DAVID T. MCARDLE

A DISSERTATION SUBMITTED IN PARTIAL FULFILLMENT OF THE REQUIREMENTS FOR THE DEGREE OF

DOCTOR OF PHILOSOPHY

IN

MATHEMATICS

UNIVERSITY OF RHODE ISLAND

2017 
DOCTOR OF PHILOSOPHY DISSERTATION

$\mathrm{OF}$

DAVID T. MCARDLE

APPROVED:

Dissertation Committee:

$\begin{array}{ll}\text { Major Professor } & \text { Orlando Merino } \\ & \text { Mustafa Kulenović } \\ & \text { Charles Kaufman } \\ & \text { Nasser H. Zawia } \\ & \text { DEAN OF THE GRADUATE SCHOOL }\end{array}$

UNIVERSITY OF RHODE ISLAND 


\begin{abstract}
Discrete dynamical systems are widely used in biological and entomological applications to model interacting populations. The manuscripts included in this thesis present global dynamic results for three different population models. Manuscript 1 presents basic concepts and definitions for general systems of difference equations in order to lay the theoretical foundation for the remaining sections.

Manuscript 2 discusses competitive systems of difference equations with the form

$$
x_{n+1}=\frac{b_{1} x_{n}}{\alpha_{1}+x_{n}+c_{1} y_{n}}, \quad y_{n+1}=\frac{b_{2} y_{n}}{\alpha_{2}+c_{2} x_{n}+y_{n}} \quad n=0,1,2, \ldots,
$$

where the parameters $b_{1}, b_{2}$ are positive real numbers and $\alpha_{1}, \alpha_{2}, c_{1}, c_{2}$ and the initial conditions $x_{0}, y_{0}$ are arbitrary nonnegative numbers. In particular, the special cases when $\alpha_{1}=\alpha_{2}=0$ and when $\alpha_{1} \neq 0$ and $\alpha_{2}=0$ are investigated. The global behavior of the system in these cases is fully characterized. Global results are also established for general competitive systems of difference equations that have a particular orientation of equilibria and certain local stability characteristics.

In Manuscript 3, the system of difference equations

$$
x_{n+1}=\frac{\alpha x_{n}}{1+\beta y_{n}}, \quad y_{n+1}=\frac{\gamma x_{n} y_{n}}{x_{n}+\delta y_{n}}, \quad n=0,1,2, \ldots
$$

is analyzed, where $\alpha, \beta, \gamma, \delta, x_{0}, y_{0}$ are positive real numbers. The system was formulated by P. H. Leslie in 1948 and models a host-parasite type of prey-predator interaction. Manuscript 3 provides the most complete dynamical analysis to date of this classic model. A boundedness and persistence result along with global attractivity results for various parameter regions are established. Numerical evidence of chaotic behavior is also presented for particular solutions of the system.
\end{abstract}


Finally, Manuscript 4 discusses structured models of difference equations with the forms:

$$
\mathrm{y}_{n+1}=M\left(f_{1}\left(\mathrm{y}_{n}^{(1)}\right), \ldots, f_{k}\left(\mathrm{y}_{n}^{(k)}\right)\right)^{t}, \quad n=0,1,2, \ldots, \quad \mathrm{y}_{0} \in \mathbb{R}_{+}^{k}
$$

and

$$
\mathrm{x}_{n+1}=A \mathrm{x}_{n}+\sum_{\ell=1}^{k} f_{\ell}\left(\mathrm{c}_{\ell} \mathrm{x}_{n}\right) \mathrm{b}_{\ell}, \quad n=0,1,2, \ldots, \quad \mathrm{x}_{0} \in X_{+},
$$

In (I) and (II), $M \in \mathbb{R}_{+}^{k \times k}, A$ is a bounded, linear operator on an ordered Banach space $X$ with positive cone $X_{+}$, and for each $\ell \in\{1, \ldots, k\}, \mathrm{b}_{\ell} \in X_{+}, \mathrm{c}_{\ell}$ is a positive, bounded linear functional on $X$, and $f_{\ell}:[0, \infty) \rightarrow[0, \infty)$ is a continuous function with $f_{\ell}(0)=0$. Conditions are established under which there is a oneto-one correspondence between positive equilibrium points (persistence states) of (I) and (II). Under these conditions, and when $X=\mathbb{R}^{m}$, the stability type of the zero equilibrium (extinction state) of (I) is shown to be the same as that for (II). Particular attention is given to the case when $k=2$. The utility of this analysis is that the dynamics of model (II) on a high dimensionality state space $X$ can be reduced to model $(\mathrm{I})$, where the dimension of the state space is the same as the number $k$ of nonlinearities that appear in (II). 


\section{ACKNOWLEDGMENTS}

I could not have successfully completed this journey on my own and there are many people that I would like to acknowledge. First and foremost, I would like to thank my PhD advisor, Dr. Orlando Merino, who has been my guide throughout my entire academic career. Not only has he been responsible for helping me grow mathematically, he has also exemplified what it means to be a passionate and committed professional. Dr. Merino has worked with me tirelessly during my time at the University of Rhode Island and he has offered me a helping hand at every step of the way.

I would also like to thank Dr. Mustafa Kulenović for offering his assistance throughout my research projects. He has sparked in me a curiosity to continue to study dynamical systems in the future and I am fortunate to have been able to learn from him over the past 9 years. Countless other professors and faculty at URI have also influenced the trajectory of my academic career and I am grateful to them as well. My fellow graduate students have also provided a great deal of support to me over the years. I have developed life-long friendships within the department that I am sure will continue as I open the next chapter of my professional career.

Finally, I would like to thank my family and friends for their moral support and encouragement throughout my graduate school experience. My wife, Wendy, has offered me unwavering support over the last eight years. I could not have completed this thesis without her constant positivity and companionship. My parents Anne and Tim, as well as my sister Beth, have also always been there to encourage me to continue to pursue my dreams and to enjoy the small victories along the way. I will forever be grateful for the guidance and encouragement that those closest to me have provided during this experience. 


\section{PREFACE}

This thesis has been written in manuscript form in accordance with the guidelines set forth by the Graduate School of the University of Rhode Island. The main content of the thesis is made up of three research papers, Manuscripts 2, 3 and 4 . Manuscript 2 was submitted for publication on March 7th, 2017 to Advances in Difference Equations, Manuscript 3 was submitted for publication on March 23rd, 2017 to the Journal of Difference Equations and Applications and Manuscript 4 will be submitted in the future. 


\section{TABLE OF CONTENTS}

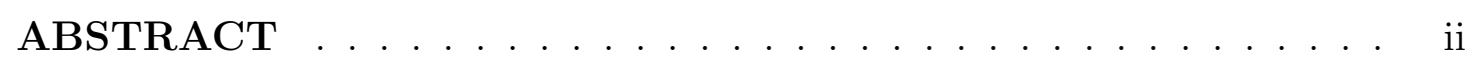

ACKNOWLEDGMENTS .................. iv

PREFACE .......................

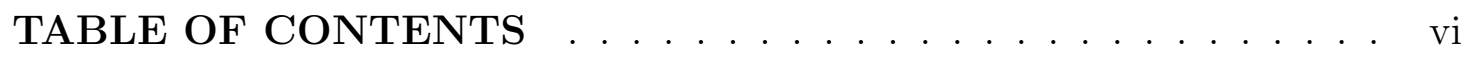

LIST OF FIGURES $\ldots \ldots \ldots \ldots \ldots \ldots \ldots \ldots \ldots$ ix

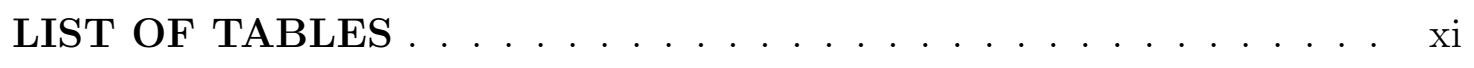

\section{MANUSCRIPT}

1 Introduction . . . . . . . . . . . . . . . . 1

1.1 Overview of Population Models . . . . . . . . . . . 2

1.2 Basic Notions of Difference Equations . . . . . . . . . . . 6

1.2.1 Local Stability Analysis _. . . . . . . . . . . 8

1.2 .2 Monotone Systems . . . . . . . . . . . . . . . 12

1.2 .3 Global Manifolds . . . . . . . . . . . . . . . 16

List of References . . . . . . . . . . . . . . . . . . 18

2 Global Dynamics of Leslie-Gower Competitive Systems in the Plane . . . . . . . . . . . . . . . . . . . 21

2.1 Introduction . . . . . . . . . . . . . . . . . 22

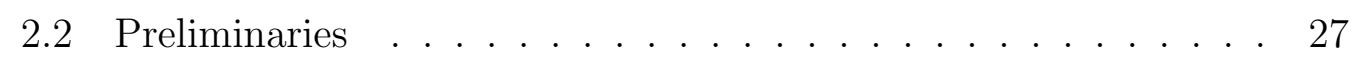

2.3 Global Dynamics of System (4) . . . . . . . . . . . 30

2.4 Global Dynamics of System (5) _ . . . . . . . . . . 38

2.5 Global Dynamics Scenarios for Competitive Systems . . . . . . 49 


\section{Page}

List of References . . . . . . . . . . . . . . . . . . . . 51

\section{The Global Dynamics and Boundedness of a Host-Parasite}

Model . . . . . . . . . . . . . . . . . . . . . . 53

3.1 Introduction . . . . . . . . . . . . . . . . . 54

3.2 Global Behavior in the Absence of a Positive Equilibrium . . . . 60

3.3 Global Attractivity of the Positive Equilibrium . . . . . . . . 63

3.4 Boundedness and Persistence of Solutions . . . . . . . . . . . 66

3.4.1 Structure of the Proof of Statement (vii) of Theorem 1 . 66

3.4.2 Ancillary Properties and Maps . . . . . . . . . . . . . . 68

3.4.3 Construction of a Family of Compact Sets . . . . . . 78

3.4.4 Proof of Proposition 3 . . . . . . . . . . . . . . 80

List of References . . . . . . . . . . . . . . . . . 86

4 Results on the Dynamics of a Class of Discrete Time Structured Population Models . . . . . . . . . . . . . . . . . 89

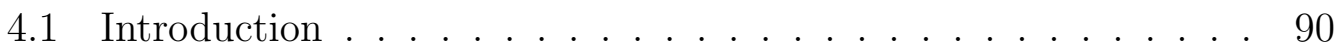

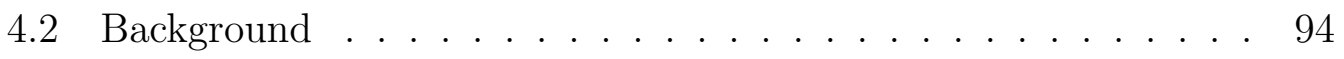

4.3 General Results for System (I) . . . . . . . . . . . . . . 96

4.4 The Two-Dimensional Case for System (I) . . . . . . . . . 101

4.4.1 Conditions for Uniqueness of the Positive Equilibrium . . 102

4.4.2 Stability of the Origin . . . . . . . . . . . . 108

4.4 .3 Examples . . . . . . . . . . . . . . . . . . . 112

4.5 Analysis of System (II) $\ldots \ldots \ldots \ldots \ldots$

4.5.1 Correspondence of Fixed Points . . . . . . . . . . . . 119

4.5.2 A Linear Algebra Result . . . . . . . . . . . . . . 122 


\section{Page}

4.5.3 Stability of the Origin _ . . . . . . . . . . . 124

4.5.4 Examples . . . . . . . . . . . . . . . 126

List of References . . . . . . . . . . . . . . . . . . . . 131

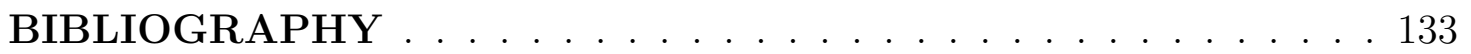




\section{LIST OF FIGURES}

Figure

Page

Global dynamics of system (1). . . . . . . . . . . . 26

Global dynamics of system (4). . . . . . . . . . . . 37

$3 \mathcal{R}_{b}:=\left\{(x, y): x, y \geq 0, c_{2} x+y \geq b_{2}\right.$ and $\left.\alpha_{1}+x+c_{1} y \leq b_{1}\right\} \quad . \quad 45$

$4 \mathcal{R}_{c}:=\left\{(x, y): x, y \geq 0\right.$ and $\left.\left(x+c_{1} y+\alpha_{1}-b_{1}\right)\left(c_{2} x+y-b_{2}\right) \leq 0\right\} 46$

5 Global dynamics of system (5). . . . . . . . . . . . . 48

6 Parameter space regions. Here "B. \& P." stands for bounded and persistent orbits, $(\bar{x}, \bar{y})$ stands for orbits converge to a unique positive equilibrium, $(\bar{x}, 0)$ stands for orbits converge to a point on the $x$-axis, and so on. . . . . . . . . . . . . . . . . 58

$7 \quad$ A plot of the orbit of $(8,8)$, for $\alpha=9999, \gamma=2$. Numerical calculations suggest $\left(x_{n}, y_{n}\right) \rightarrow(\bar{x}, \bar{y})=(9000,9000)$. In plot $(\mathrm{a})$, the equilibrium $(9000,9000)$ is shown as a black dot. Plot (b) shows a smaller window, where some lune-shaped regions apparently devoid of points can be seen. One such region seems to contain the point $(3500,1900)$, which is marked with the symbol + . A total of 300000 points were generated for these plots. . . . . . . . . . 59

8 The orbit of $(3500,1900)$, for $\alpha=9999, \gamma=2$ seems to be a subset of 10 connected compact sets, none of which contains the unique positive equilibrium. Compare to Figure 7. The positive equilibrium $(9000,9000)$ is shown as an isolated dot. A total of 60000 points were generated for this plot. . . . . . . . . . . . 59

$9 \quad$ (a) The sets $\mathcal{P}_{2}$ and $\mathcal{P}_{4}$. (b) The boundary of a set $\mathcal{P}_{\mu}$ (solid) and its image $S\left(\partial \mathcal{P}_{\mu}\right)$ (dashed). . . . . . . . . . 64

10 Equilibrium curves and complementary regions for $T$ and $\hat{T}$, respectively. ................... 70

11 The curve $\hat{\mathcal{D}}_{1}^{\prime}$ (thick, solid) and its image under $\hat{T}$ (thick, dashed) 72

12 The curve $\hat{\mathcal{D}}_{2}^{\prime}$ (solid) and its image under $\hat{T}$ (dashed) . . . . . . 74 
13 The curve $\hat{\mathcal{D}}_{3}^{\prime}$ (solid) and its image under $\hat{T}$ (dashed) . . . . . 76

$14 \quad \mathrm{~A}$ set $\mathcal{K}_{\tau}$ whose boundary consists of the sets $\hat{\mathcal{D}}_{\ell}^{\prime}$ for $0 \leq \ell \leq 4.79$

15 The quadrilateral $\mathcal{S}_{\tau}$ with $\mathcal{S}_{\tau} \subset \mathcal{K}_{\tau} \ldots \ldots$. . . . . . . . 81

$16 \quad$ Boundary of the set $\mathcal{K}_{\tau}$ (solid) and its image under $\hat{T}$ (dashed). $\quad 82$

17 The parametric curves $\phi_{1}$ and $\phi_{2}$ are plotted for various situations described in Theorem 4. In (a) conditions (A), (B1) and (B2) are satisfied, in (b) (A) and (B2) are satisfied, in (c) (A) and (B1) are satisfied and in (d) (A) and (B3) are satisfied. . . 104

18 The parametric curves $\phi_{1}$ and $\phi_{2}$ are plotted for different cases described in Theorem 5. In figure (a) conditions (B1) and (C2) are satisfied, in (b) (B2) and (C1) are satisfied, in (c) (D) is satisfied and in (d) (B2) and (C2) are satisfied. Note that hypothesis (A) is satisfied in all cases. . . . . . . . . . . . 108

19 The curves $\phi_{1}$ and $\phi_{2}$ corresponding to system (62) can be seen in (a). A solution $\left(x_{n}, y_{n}\right)$ to $(62)$ with $\left(x_{0}, y_{0}\right)=(2,6)$ can be seen in (b). The solution quickly approaches the equilibrium point $\bar{x}$ given in (63) . . . . . . . . . . . . . . . 128

20 The curves $\phi_{1}$ and $\phi_{2}$ corresponding to system (64) can be seen in (a). The global stable and unstable manifolds, $\mathcal{W}^{s}$ and $\mathcal{W}^{u}$, corresponding to the interior saddle point $\overline{\mathrm{x}}$ can be seen in (b). . 130

21 A solution $\left(x_{n}, y_{n}\right)$ to $(64)$ with $\left(x_{0}, y_{0}\right)=\left(\frac{3}{4}, \frac{1}{4}\right)$ can be seen in (a). The solution quickly converges to the origin. A solution $\left(x_{n}, y_{n}\right)$ to $(64)$ with $\left(x_{0}, y_{0}\right)=(1,1)$ can be seen in $(\mathrm{b})$. The solution approaches $(+\infty,+\infty)$. . . . . . . . . . 130 


\section{LIST OF TABLES}

Table

$1 \quad$ Global behavior of solutions to (1) when $b_{1}>1$ and $b_{2}>1$. Equality relations are not represented for the sake of a simpler description. . . . . . . . . . . . . . . . 23

2 The equilibrium points for system (4). . . . . . . . . . . . 32

3 The equilibrium points for system (5). . . . . . . . . . . . 40 


\section{MANUSCRIPT 1}

\section{Introduction}

The dynamical behavior of biological populations has been a primary focus of applied mathematicians for hundreds of years. Modeling and predicting population growth can be tracked back to 1202 when Leonardo Fibonacci considered the progression of a hypothetical rabbit population in his book Liber Abaci $[1,2]$. These models grew more complex in 1798 when Thomas Malthus presented the Malthusian exponential growth model and in 1844 when Pierre François Verhulst introduced the logistic model of population growth $[1,2]$. The theory in the area of population dynamics has seen steady progress and many complicated biological phenomena have been accurately modeled. These models are referred to as dynamical systems. Dynamical systems mathematically describe the temporal progression of a given quantity and they come in the form of both difference equations and differential equations [3].

Differential equations are continuous dynamical systems that account for the behavior of a system at all times and are useful in modeling phenomena that are continuously evolving [3]. Difference equations, on the other hand, are discrete dynamical systems that are useful when describing populations with non-overlapping generations. These discrete models often exhibit unique dynamical scenarios that cannot otherwise be modeled by differential equations and can more accurately reflect the global character of certain populations. See [4], [5], [6], and [7] for interesting applications.

The focus of this thesis is on systems of difference equations and their applications to population dynamics. In particular, several population models are analyzed and results are presented related to the global dynamics of these sys- 
tems. The remainder of the introduction is organized as follows: an overview of the types of population models models that are studied in Manuscripts 2, 3, and 4 is presented in Section 1.1 along with a summary of the important results that are attained. Basic theory of difference equations is then presented in Section 1.2, which helps to lay the theoretical foundation for the proceeding manuscripts.

\subsection{Overview of Population Models}

The population models that are studied within this thesis include competitive models, host-parasitoid and host parasite models, and structured population models. Background for each of these models is presented in this section. An overview of the specific results that are attained in Manuscripts 2, 3, and 4 is also provided.

A two-dimensional competitive system of difference equations has the form

$$
x_{n+1}=f\left(x_{n}, y_{n}\right), \quad y_{n+1}=g\left(x_{n}, y_{n}\right), \quad n=0,1,2, \ldots,
$$

where the function $f$ is nondecreasing in the first variable and nonincreasing in the second and $g$ is nonincreasing in the first variable and nondecreasing in the second [8]. Systems of this type can be used to model two species living in an environment that are in competition for the same resources. Competitive systems satisfy monotonicty properties (as discussed in Section 1.2.2), which are useful

in proving global results. See [9] and [10] for interesting competitive systems of difference equations that have been studied recently. There are many dynamical scenarios that can exist for a competitive system, including competitive exclusion, competitive coexistence, and Allee's effect. Competitive exclusion occurs when one species is driven to extinction, competitive coexistence occurs when two competing species reach an equilibrium state and are able to coexist, and Allee's effect occurs when two species with nonzero populations are both driven to extinction [11].

Many of the original two-species, discrete competition models were based off of the Lotka-Volterra competitive system of differential equations and they exhibited 
primarily the phenomenon of competitive exclusion [12]. One of the first models that incorporated competitive coexistence was formulated by J. C. Gower and P. H. Leslie in 1958 [13]. Based on experiments performed by T. Park in 1948 [14], Leslie and Gower presented the competitive model

$$
\begin{aligned}
& N_{1}(t+1)=\frac{\lambda_{1} N_{1}(t)}{1+\alpha_{1} N_{1}(t)+\beta_{1} N_{2}(t)}, \\
& N_{2}(t+1)=\frac{\lambda_{2} N_{2}(t)}{1+\alpha_{2} N_{2}(t)+\beta_{2} N_{1}(t)}, \quad t=0,1,2 \ldots,
\end{aligned}
$$

where $\lambda_{1}, \lambda_{2}, \alpha_{1}, \alpha_{2}, \beta_{1}, \beta_{2}$ are positive constants. Manuscript 2 focuses on a comparable form of system (1), given by

$$
x_{n+1}=\frac{b_{1} x_{n}}{\alpha_{1}+x_{n}+c_{1} y_{n}}, \quad y_{n+1}=\frac{b_{2} y_{n}}{\alpha_{2}+c_{2} x_{n}+y_{n}}, \quad n=0,1,2, \ldots
$$

where the parameters $b_{1}, b_{2}$ are positive real numbers, and $\alpha_{1}, \alpha_{2}, c_{1}, c_{2}$ and the initial conditions $x_{0}, y_{0}$ are arbitrary nonnegative numbers. The case when all parameters are taken to be positive was studied in [12] by J. M. Cushing. The analysis in [12] is not complete, however, as it does not treat the sensitive case when $\alpha_{1}=0$ or $\alpha_{2}=0$. In these cases, the extinction equilibrium is replaced by a singular point at the origin, which greatly complicates the problem. Manuscript 2 fully characterizes the global dynamics of this system when $\alpha_{1}=0$ or $\alpha_{2}=0$ and the dynamical scenarios are compared to the case when $\alpha_{1}, \alpha_{2}>0$. The proof techniques reflect the sensitivity of the singular point at the origin. Using these techniques, global results are also established for general competitive systems of difference equations that have a particular orientation of equilibria (or singular points) and certain local stability characteristics.

A host-parasitoid model is a type of prey-predator model involving two species, a parasitoid and a host [3]. A parasitoid is a type of parasite that lives freely and lays eggs in the larvae or pupae of the host population. The development of the parasitoid depends on the population of the host and the population of the host 
depends on how many of its peers survived the infestation [3, 15]. The general framework for describing the dynamics of such a model is

$$
x_{n+1}=a x_{n} f\left(x_{n}, y_{n}\right), \quad y_{n+1}=c x_{n}\left(1-f\left(x_{n}, y_{n}\right)\right), \quad n=0,1,2, \ldots,
$$

where $x_{n}$ and $y_{n}$ represent the size of the host and parasitoid populations in the $n^{\text {th }}$ generation, respectively, and $f\left(x_{n}, y_{n}\right)$ represents the fraction of hosts that survive the parasitoid $[15,16]$. One of the first models of this type was developed by Nicholson and Bailey in 1935 for a host, Trialeurodes vaporariorum, and a parasitoid, Encarsia formosa, and is described by the system

$$
x_{n+1}=a x_{n} e^{-b y_{n}}, \quad y_{n+1}=x_{n}\left(1-e^{-b y_{n}}\right), \quad n=0,1,2, \ldots
$$

This model assumes that the reproductive rate of the host and the searching efficiency of the parasitoid are constant and that the environment is consistent for all parasitoids $[16,17]$. A host-parasite model has a similar structure to that of a host-parasitoid model but the parasite may not kill the host [3, 18]. One hostparasite model of particular interest, formulated in 1948 by P. H. Leslie, is given by

$$
\begin{aligned}
& N_{1}(t+1)=\frac{\lambda_{1} N_{1}(t)}{1+\left(\lambda_{1}-1\right) \frac{N_{2}(t)}{K_{2}}}, \\
& N_{2}(t+1)=\frac{\lambda_{2} N_{2}(t)}{1+\left(\lambda_{2}-1\right) \frac{K_{1} N_{2}(t)}{K_{2} N_{1}(t)}}, \quad t=0,1,2, \ldots,
\end{aligned}
$$

where $\lambda_{1}, \lambda_{2}>1$ and $K_{1}, K_{2}$ are positive constants (see page 239 in [19]). Manuscript 3 takes a closer look at system (2) and presents the most complete analysis to date of this classic model. A boundedness and persistence result along with global attractivity results for various parameter regions are established. Numerical evidence of chaotic behavior is also presented for particular solutions of the system. 
The third type of population model that is analyzed in this thesis is referred to as a structured population model. Structured models are useful for populations that include individuals with a variety of physical or physiological characteristics that may interact with the environment differently [4]. These differences play an important role in the dynamics of the entire population and must be taken into account within the model. The basic theory for models of this type is presented by J. M. Cushing in [2]. The general framework involves dividing a population into $k$ classes so that at discrete time $n \in \mathbb{N}$ the number of individuals (or density) in class $\ell$ is $\mathrm{y}_{n}^{(\ell)}$. The vector $\mathrm{y}_{n} \in \mathbb{R}_{+}^{k}$, defined by

$$
\mathrm{y}_{n}=\left(\mathrm{y}_{n}^{(1)}, \mathrm{y}_{n}^{(2)}, \ldots, \mathrm{y}_{n}^{(k)}\right)
$$

tracks the size of each of the $k$ classes. It is typically assumed that the new number of individuals in each class is a function of the previous number of individuals in each of the $k$ classes [2]. This inter-dependence can be condensed in the matrix model:

$$
\mathrm{y}_{n+1}=P\left(\mathrm{y}_{n}\right) \mathrm{y}_{n}, \quad n=0,1,2, \ldots, \quad \mathrm{y}_{0} \in \mathbb{R}_{+}^{k},
$$

which is explored in [2]. The operator $P$ is referred to as a projection matrix. Manuscript 4 focuses on a special case of systems of the form (3), given by

$$
\mathrm{y}_{n+1}=M\left(f_{1}\left(\mathrm{y}_{n}^{(1)}\right), \ldots, f_{k}\left(\mathrm{y}_{n}^{(k)}\right)\right)^{t}, \quad n=0,1,2, \ldots, \quad \mathrm{y}_{0} \in \mathbb{R}_{+}^{k},
$$

where $M \in \mathbb{R}_{+}^{k \times k}$ and for $\ell \in\{1, \ldots, k\}, f_{\ell}:[0, \infty) \rightarrow[0, \infty)$ is a continuous function with $f_{\ell}(0)=0$. System (I) is similar to a genetics model formulated by Friedland and Karlin in [20]. Results are established in Manuscript 4 related to the existence, uniqueness, and stability of a positive equilibrium (i.e. persistence state) as well as to the stability of the origin (i.e. extinction state). Particular attention is given to the special case when $k=2$. The benefit of analyzing system (I) is that it can be used as a tool to study more complicated structured population 
models, given by

$$
\mathrm{x}_{n+1}=A \mathrm{x}_{n}+\sum_{\ell=1}^{k} f_{\ell}\left(\mathrm{c}_{\ell} \mathrm{x}_{n}\right) \mathrm{b}_{\ell}, \quad n=0,1,2, \ldots, \quad \mathrm{x}_{0} \in X_{+},
$$

where $A$ is a bounded, linear operator on an ordered Banach space $X$ with positive cone $X_{+}$, and for each $\ell \in\{1, \ldots, k\}, \mathrm{b}_{\ell} \in X_{+}$and $\mathrm{c}_{\ell}$ is a positive bounded linear functional on $X$. Model (II) is a generalization of a model studied by Rebarber, Townley, and Tenhumberg in [21] and can be useful to model plant and fishery populations. The matrix $A$ is referred to as the survival operator and it reflects survival and growth of each class. The terms $f_{\ell}(\cdot) \mathrm{b}_{\ell}$ are referred to as fecundity operators and they reflect the nonlinear dependence on new offspring and the redistribution of the offspring to each class of the structured model [21].

Model (II) is potentially set in a high dimensionality state space $X$. The nonlinearities, however, are of a very specific type. Manuscript 4 presents a technique to greatly reduce the complexity of the problem to one where the dimension of the state space is the same as the number $k$ of nonlinearities that appear in (II). In particular, a $k$-dimensional model of the form (I) is found to have a significant connection with (II). Conditions are given under which there is a one-to-one correspondence between the positive equilibrium points of (I) and (II). Furthermore, when the state space of (II) is $\mathbb{R}^{m}$, it is established that the stability character of the origin in (I) is the same as that of (II). In this way, all of the results established for system (I) can be used to establish results for (II).

For convenience to the reader, basic theory and results are presented in the coming sections that contribute to establishing global results for the three models introduced above.

\subsection{Basic Notions of Difference Equations}

Difference equations are used to describe the progression of a given quantity over discrete time increments. As mentioned above, a common application is 
tracking the size of a population with discrete generations. If we denote by $x_{n}$ the size of the population in the $n^{\text {th }}$ generation and assume that the size of the population in the $n+1^{\text {st }}$ generation (i.e. $x_{n+1}$ ) is a function of $x_{n}$, we arrive at the difference equation

$$
x_{n+1}=f\left(x_{n}\right), \quad n=0,1,2, \ldots
$$

If the initial value of the population were known ( say $x_{0}$ ), then (4) provides all of the information needed to track the population through each generation [22, 23]. The resulting sequence $\left\{x_{0}, f\left(x_{0}\right), f\left(f\left(x_{0}\right)\right), f\left(f\left(f\left(x_{0}\right)\right)\right), \ldots\right\}$ is called a solution of (4). This type of difference equation is first-order ( since $x_{n+1}$ only depends on one previous generation) and autonomous (since $x_{n+1}$ does not depend explicitly on $n)[22,23]$.

Of particular interest in this thesis are systems of difference equations modeling two or more populations that depend on one another through each discrete generation. Consider a planar region $\mathcal{D} \in \mathbb{R}^{2}$. A two-dimensional, first-order system of difference equations has the form

$$
x_{n+1}=f\left(x_{n}, y_{n}\right), \quad y_{n+1}=g\left(x_{n}, y_{n}\right), \quad n=0,1,2, \ldots
$$

where $f, g: \mathcal{D} \rightarrow \mathbb{R}$ are continuous functions and $\left(x_{0}, y_{0}\right) \in \mathcal{D}$. Systems of this form can be used to model two populations that interact in many different ways, including cooperation, competition, and predator-prey type interactions. Associated with system (5) is a continuous map $T: \mathcal{D} \rightarrow \mathcal{D}$, where

$$
T(u, v)=\left(\begin{array}{l}
f(u, v) \\
g(u, v)
\end{array}\right)
$$

If we specify an initial condition $\left(x_{0}, y_{0}\right) \in \mathcal{D}$ and repeatedly apply the map $T$, the resulting sequence of ordered pairs $\left\{\left(x_{0}, y_{0}\right), T\left(x_{0}, y_{0}\right), T^{2}\left(x_{0}, y_{0}\right), T^{3}\left(x_{0}, y_{0}\right), \ldots\right\}$ is called a solution of (5) (see $[22,23])$. 
If one were to find a solution of a difference equation by specifying initial conditions, the main question to consider is how the resulting sequence behaves as $n \rightarrow \infty$. A complete description of the behavior of the solutions for an arbitrary initial condition is referred to as the global behavior of the difference equation. This analysis begins with finding the equilibrium points of the system. An equilibrium point $(\bar{x}, \bar{y})$ of $(5)$ is a point that satisfies $\bar{x}=f(\bar{x}, \bar{y})$ and $\bar{y}=g(\bar{x}, \bar{y})$. Equilibrium points of (5) are referred to as fixed points of $(6)$ where $(\bar{x}, \bar{y})=T(\bar{x}, \bar{y})$. For each fixed point, the basin of attraction $\mathcal{B}(\bar{x}, \bar{y})$ is the largest set of points in $\mathcal{D}$ that are attracted to $(\bar{x}, \bar{y})$ under forward iterations of $T$ (see [23]). That is,

$$
\mathcal{B}(\bar{x}, \bar{y})=\left\{(x, y) \in \mathcal{D}: T^{n}(x, y) \rightarrow(\bar{x}, \bar{y}) \quad \text { as } \quad n \rightarrow \infty\right\}
$$

There is also the potential for the existence of periodic solutions, which play a role in the global behavior of system (5). A minimal period-two solution is a point $(x, y) \in \mathcal{D}$ such that $T^{2}(x, y)=(x, y)$ and $T(x, y) \neq(x, y)[22,23]$. The same principles described above can also be applied to larger dimensional systems.

The allure of difference equations is that the global behavior of a system can be extremely simple or incredibly complex. There can be multiple equilibrium points, periodic points, attracting curves, unbounded behavior, and even chaos. Determining global behavior is the central focus of much of the research that is done in difference equations. The following subsections provide some of the more specific theory that is used to establish global results.

\subsubsection{Local Stability Analysis}

To determine the global behavior of a system of difference equations, the first step is understanding the local behavior in a neighborhood of each equilibrium point. This analysis is referred to as local stability analysis. Consider the two-

dimensional system of difference equations given in (5). Let $\|\cdot\|$ denote the euclidean norm in $\mathbb{R}^{2}$. That is, $\|(x, y)\|=\sqrt{x^{2}+y^{2}}$. The following definitions 
can be seen in their original form in introductory textbooks written by M. R. S. Kulenović and O. Merino [23] or S. Elaydi [22]. The definitions are presented for a two-dimensional system of differences equations, but the same principles apply for higher dimension.

Definition 1 Consider an equilibrium point $(\bar{x}, \bar{y})$ of system (5). Then

(i) $(\bar{x}, \bar{y})$ is locally stable if for any $\epsilon>0$ there exists $\delta>0$ such that for every initial point $\left(x_{0}, y_{0}\right)$ with $\left\|\left(x_{0}, y_{0}\right)-(\bar{x}, \bar{y})\right\|<\delta$, the iterates $\left(x_{n}, y_{n}\right)$ satisfy $\left\|\left(x_{n}, y_{n}\right)-(\bar{x}, \bar{y})\right\|<\epsilon$ for all $n>0$.

(ii) $(\bar{x}, \bar{y})$ is locally asymptotically stable if, in addition to being stable, $\left(x_{n}, y_{n}\right) \rightarrow$ $(\bar{x}, \bar{y})$ as $n \rightarrow \infty$ for all $\left(x_{0}, y_{0}\right)$ that satisfy $\left\|\left(x_{0}, y_{0}\right)-(\bar{x}, \bar{y})\right\|<\delta$.

Consider the map $T: \mathcal{D} \rightarrow \mathcal{D}$ given in (6), where $f$ and $g$ are taken to be continuously differentiable on $\mathcal{D}$. In order to determine the local character of a fixed point, one can consider the linearization of the map near each fixed point. This requires computing the Jacobian matrix of $T$ at $(\bar{x}, \bar{y})$, which is given by

$$
J_{T}(\bar{x}, \bar{y})=\left(\begin{array}{ll}
\frac{\partial f}{\partial x}(\bar{x}, \bar{y}) & \frac{\partial f}{\partial y}(\bar{x}, \bar{y}) \\
\frac{\partial g}{\partial x}(\bar{x}, \bar{y}) & \frac{\partial g}{\partial y}(\bar{x}, \bar{y})
\end{array}\right) .
$$

The linearization of the map $T$, denoted by $D_{T}$, at the fixed point $(\bar{x}, \bar{y})$ is then given by

$$
D_{T}(\bar{x}, \bar{y})=\left(\begin{array}{ll}
\frac{\partial f}{\partial x}(\bar{x}, \bar{y}) & \frac{\partial f}{\partial y}(\bar{x}, \bar{y}) \\
\frac{\partial g}{\partial x}(\bar{x}, \bar{y}) & \frac{\partial g}{\partial y}(\bar{x}, \bar{y})
\end{array}\right)\left(\begin{array}{l}
x \\
y
\end{array}\right),
$$

and the characteristic equation associated with the Jacobian matrix (7) is

$$
\lambda^{2}-\operatorname{tr}\left(J_{T}(\bar{x}, \bar{y})\right) \lambda+\operatorname{det}\left(J_{T}(\bar{x}, \bar{y})\right)=0 .
$$

Locally, the map $T$ behaves like the linearization given in (8). Therefore, the eigenvalues $\lambda_{1}$ and $\lambda_{2}$ of $J_{T}(\bar{x}, \bar{y})$ (i.e. roots of the characteristic equation) provide information about the local stability characteristics of $(\bar{x}, \bar{y})$. If both eigenvalues 
have modulus less than one, then $(\bar{x}, \bar{y})$ is locally asymptotically stable and if at least one of the eigenvalues is greater than one in modulus, then $(\bar{x}, \bar{y})$ is unstable. Furthermore, a fixed point $(\bar{x}, \bar{y})$ of the map $T$ is hyperbolic if $J_{T}(\bar{x}, \bar{y})$ has no eigenvalues on the unit circle, otherwise $(\bar{x}, \bar{y})$ is said to be nonhyperbolic. Hyperbolic fixed points can have three qualitatively distinct classifications, which are described in Definition 2.

Definition 2 Consider an equilibrium point $(\bar{x}, \bar{y})$ of system (5),

(i) If $(\bar{x}, \bar{y})$ is locally asymptotically stable then the eigenvalues of $J_{T}(\bar{x}, \bar{y})$ are such that $\left|\lambda_{1}\right|,\left|\lambda_{2}\right|<1$. In this case, there is an open neighborhood $U$ of $(\bar{x}, \bar{y})$ in which all points converge to the equilibrium under forward iterations of the map T. That is,

$$
T^{n}(x, y) \rightarrow(\bar{x}, \bar{y}) \quad \text { for all } \quad(x, y) \in U
$$

Such an equilibrium point is referred to as a sink. Parts (ii) and (iii) describe the two unstable situations.

(ii) If the eigenvalues of $J_{T}(\bar{x}, \bar{y})$ are such that $\left|\lambda_{1}\right|,\left|\lambda_{2}\right|>1$, then there is an open neighborhood $U$ of $(\bar{x}, \bar{y})$ in which all points converge to the equilibrium point under backward iterations of the map T. That is,

$$
T^{-n}(x, y) \rightarrow(\bar{x}, \bar{y}) \quad \text { for all } \quad(x, y) \in U
$$

Such an equilibrium point is referred to as a source or repeller.

(iii) If the eigenvalues of $J_{T}(\bar{x}, \bar{y})$ are such that $\left|\lambda_{1}\right|<1$ and $\left|\lambda_{2}\right|>1$, then in any neighborhood $U$ of $(\bar{x}, \bar{y})$, the forward iterates under $T$ of some points in $U$ converge to $(\bar{x}, \bar{y})$ and the backward iterates under $T$ of some points in $U$ converge to $(\bar{x}, \bar{y})$. Such a point is referred to as a saddle point. 
There are shortcuts that can be used to determine the local character of equilibrium points in the two dimensional case, referred to as the Schur-Cohn Criteria.

Theorem 1 The following conditions hold for equilibrium points $(\bar{x}, \bar{y})$ of system (5):

(i) An equilibrium point $(\bar{x}, \bar{y})$ is locally asymptotically stable if and only if

$$
\left|\operatorname{tr} J_{T}(\bar{x}, \bar{y})\right|<1+\operatorname{det} J_{T}(\bar{x}, \bar{y})<2 \text {. }
$$

(ii) An equilibrium point $(\bar{x}, \bar{y})$ is locally a repeller if and only if

$$
\left|\operatorname{tr} J_{T}(\bar{x}, \bar{y})\right|<\left|1+\operatorname{det} J_{T}(\bar{x}, \bar{y})\right| \quad \text { and } \quad\left|\operatorname{det} J_{T}(\bar{x}, \bar{y})\right|>1 .
$$

(iii) An equilibrium point $(\bar{x}, \bar{y})$ is locally a saddle point if and only if

$$
\left|\operatorname{tr} J_{T}(\bar{x}, \bar{y})\right|>\left|1+\operatorname{det} J_{T}(\bar{x}, \bar{y})\right| \quad \text { and } \quad \operatorname{tr} J_{T}(\bar{x}, \bar{y})^{2}-4 \operatorname{det} J_{T}(\bar{x}, \bar{y})>0 \text {. }
$$

(iv) An equilibrium point $(\bar{x}, \bar{y})$ is nonhyperbolic if and only if

$$
\left|\operatorname{tr} J_{T}(\bar{x}, \bar{y})\right|=\left|1+\operatorname{det} J_{T}(\bar{x}, \bar{y})\right| \quad \text { or } \quad\left|\operatorname{tr} J_{T}(\bar{x}, \bar{y})\right| \leq 2 \text { and } \operatorname{det} J_{T}(\bar{x}, \bar{y})=1 .
$$

In the case of a nonhyperbolic equilibrium (i.e. when eigenvalues of $J_{T}(\bar{x}, \bar{y})$ satisfy $\left|\lambda_{j}\right|=1$ for $j=1$ or 2 ), more analysis is needed to determine the local stability character. This analysis involves higher order terms in the Taylor expansion, which will not be covered here.

Another theorem that is helpful in analyzing eigenvalues of the Jacobian matrix is the Perron-Frobenius Theorem from [24]. Prior to stating the theorem, a few basic notions of matrices are needed: A matrix $A \in \mathbb{R}^{m \times m}$ is nonnegative (positive), written $A \geq 0(A>0)$ if all of the entries of $A$ are nonnegative (positive). 
A nonnegative matrix $A$ is called primitive if there exists an $N \in \mathbb{N}$ such that $A^{N}>0$. The matrix $A$ is irreducible if for any $i, j$ there is a $k=k(i, j)$ such that $\left(A^{k}\right)_{i j}>0$ [24]. The Perron-Frobenius Theorem specifically treats nonnegative, irreducible matrices.

Theorem 2 Let $A \in \mathbb{R}^{m \times m}$ be nonnegative and irreducible. Then,

(i) A has a positive (real) eigenvalue $\lambda_{\max }$ such that all other eigenvalues of $A$ satisfy $|\lambda| \leq \lambda_{\max }$.

(ii) $\lambda_{\max }$ has algebraic and geometric multiplicity one, and has an eigenvector $\mathrm{x}>0$.

(iii) Any nonnegative eigenvector is a multiple of $\mathrm{x}$.

(iv) Suppose $\mathrm{y} \in \mathbb{R}_{+}^{m}, \mathrm{y} \neq 0$ and $\mu \in \mathbb{R}$ is such that $A \mathrm{y} \leq \mu \mathrm{y}$. Then $\mathrm{y}>0$ and $\mu \geq \lambda_{\max }$, with $\mu=\lambda_{\max }$ if and only if $\mathrm{y}$ is a multiple of $\mathrm{x}$.

(v) If $0 \leq B \leq A, B \neq A$, then every eigenvalue $\sigma$ of $B$ satisfies $|\sigma|<\lambda_{\max }$.

(vi) If $A$ is primitive, then all other eigenvalues of $A$ satisfy $|\lambda|<\lambda_{\max }$

In the following section, basic notions of monotone maps are presented, which can play a critical role in determining the global dynamics of systems of difference equations.

\subsubsection{Monotone Systems}

A set $\mathcal{P} \subset \mathbb{R}^{m}$ is an order cone if $\mathcal{P}$ is closed, convex, and such that $\lambda \mathcal{P} \subset \mathcal{P}$ for all $\lambda \geq 0, \mathcal{P} \cap(-\mathcal{P})=\{0\}$ and $\mathcal{P} \neq\{0\}$. Every order cone $\mathcal{P}$ induces a partial order $\preceq$ on $\mathbb{R}^{m}$. For points $\mathrm{x}, \mathrm{y} \in \mathcal{P}$, we say $\mathrm{x} \preceq \mathrm{y}$ if and only if $\mathrm{y}-\mathrm{x} \in \mathcal{P}, \mathrm{x} \prec \mathrm{y}$ if and only if $\mathrm{y}-\mathrm{x} \in \mathcal{P} \backslash\{0\}$, and $\mathrm{x} \ll \mathrm{y}$ if and only if $\mathrm{y}-\mathrm{x} \in \operatorname{int}(\mathcal{P})$. The 
ordered set $\llbracket \mathrm{x}, \mathrm{y} \rrbracket$ relative to the partial order $\preceq$ is defined by

$$
\llbracket \mathrm{x}, \mathrm{y} \rrbracket:=\left\{\mathrm{u} \in \mathbb{R}^{m}: \mathrm{x} \preceq \mathrm{u} \preceq \mathrm{y}\right\}
$$

For $\mathcal{D} \subset \mathbb{R}^{m}$, a map $T: \mathcal{D} \rightarrow \mathcal{D}$ is said to be monotone (with respect to the partial order $\preceq$ ) if $\mathrm{x} \preceq \mathrm{y}$ implies that $T(\mathrm{x}) \preceq T(\mathrm{y})$. The map $T$ is strictly monotone if $\mathrm{x} \prec$ $\mathrm{y}$ implies that $T(\mathrm{x}) \prec T(\mathrm{y})$ and strongly monotone if $\mathrm{x} \prec \mathrm{y}$ implies that $T(\mathrm{x}) \ll$ $T(y)$. See [8] for more detailed information regarding monotone systems. The following result is stated for monotone (i.e. order preserving) maps and appears in $[25]$.

Theorem 3 For a nonempty set $\mathcal{D} \in \mathbb{R}^{m}$ and a partial order $\preceq$ on $\mathbb{R}^{m}$, let $T$ : $\mathcal{D} \rightarrow \mathcal{D}$ be an order-preserving map and let $\mathrm{u}, \mathrm{v} \in \mathcal{D}$ be such that $\mathrm{u} \prec \mathrm{v}$ and $\llbracket \mathrm{u}, \mathrm{v} \rrbracket \subset \mathcal{D}$. If $\mathrm{u} \preceq T(\mathrm{u})$ and $T(\mathrm{v}) \preceq \mathrm{v}$, then $\llbracket \mathrm{u}, \mathrm{v} \rrbracket$ is an invariant set and

(i) There exists a fixed point of $T$ in $\llbracket \mathrm{u}, \mathrm{v} \rrbracket$.

(ii) If $T$ is strongly order-preserving then there exists a fixed point of $T$ in $\llbracket \mathrm{u}, \mathrm{v} \rrbracket$ that is stable relative to $\llbracket \mathrm{u}, \mathrm{v} \rrbracket$.

(iii) If there is only one fixed point in $\llbracket \mathrm{u}, \mathrm{v} \rrbracket$ then it is a global attractor in $\llbracket \mathrm{u}, \mathrm{v} \rrbracket$ and therefore asymptotically stable relative to $\llbracket \mathrm{u}, \mathrm{v} \rrbracket$.

A direct consequence of Theorem 3 is Corollary 1 proven by Dancer and Hess in $[25]$.

Corollary 1 If the nonnegative cone of a partial ordering $\preceq$ is a generalized orthant in $\mathbb{R}^{m}$, and if $T$ has no fixed points in $\llbracket \mathrm{u}, \mathrm{v} \rrbracket$ other than $\mathrm{u}$ and $\mathrm{v}$, then the interior of $\llbracket \mathrm{u}, \mathrm{v} \rrbracket$ is either a subset of the basin of attraction of $\mathrm{u}$ or the basin of attraction of $\mathrm{v}$.

If we restrict out attention to $\mathbb{R}^{2}$, then there are two standard partial orders, the North-East and South-East partial orders [8]. 
Definition 3 The North-East partial order $\preceq_{n e}$ on $\mathbb{R}^{2}$ is defined as follows:

$$
\left(\begin{array}{l}
x_{1} \\
y_{1}
\end{array}\right) \preceq_{n e}\left(\begin{array}{l}
x_{2} \\
y_{2}
\end{array}\right) \Leftrightarrow\left\{\begin{array}{l}
x_{1} \leq x_{2} \\
y_{1} \leq y_{2}
\end{array},\right.
$$

where the positive cone is taken to be the standard first quadrant. The South-East partial order $\preceq_{\text {se }}$ on $\mathbb{R}^{2}$ is defined as follows:

$$
\left(\begin{array}{l}
x_{1} \\
y_{1}
\end{array}\right) \preceq_{s e}\left(\begin{array}{l}
x_{2} \\
y_{2}
\end{array}\right) \Leftrightarrow\left\{\begin{array}{l}
x_{1} \leq x_{2} \\
y_{1} \geq y_{2}
\end{array}\right.
$$

where the positive cone is taken to be the standard fourth quadrant.

The partial-orders in Definition 3 are associated with two important monotone maps on $\mathbb{R}^{2}$. For $\mathcal{D} \subset \mathbb{R}^{2}$, a map $T: \mathcal{D} \rightarrow \mathcal{D}$ is competitive if it is nondecreasing with respect to the South-East partial order $\preceq_{s e}$. In other words:

$$
\left(\begin{array}{l}
x_{1} \\
y_{1}
\end{array}\right) \preceq_{s e}\left(\begin{array}{l}
x_{2} \\
y_{2}
\end{array}\right) \Rightarrow T\left(\begin{array}{l}
x_{1} \\
y_{1}
\end{array}\right) \preceq_{s e} T\left(\begin{array}{l}
x_{2} \\
y_{2}
\end{array}\right) \text {. }
$$

The map $T$ is strongly competitive if it is strictly increasing with respect to the South-East partial order [8]. A sufficient condition for $T$ to be strongly competitive is that the Jacobian matrix associated with $T$ has the sign configuration

$$
\left(\begin{array}{ll}
+ & - \\
- & +
\end{array}\right)
$$

Similarly, $T$ is said to be cooperative if it is nondecreasing with respect to the North-East partial order $\preceq_{n e}$ and strongly cooperative if it is strictly increasing with respect to the North-East partial order [8]. A sufficient condition for $T$ to be strongly cooperative is that the Jacobian matrix associated with $T$ has the sign configuration

$$
\left(\begin{array}{ll}
+ & + \\
+ & +
\end{array}\right)
$$


The following definition presents another important property that competitive maps can satisfy on $\mathbb{R}^{2}[8]$.

Definition 4 Suppose $\mathcal{D} \subset \mathbb{R}^{2}$ is nonempty. A competitive map $T: \mathcal{D} \rightarrow \mathcal{D}$

(i) Satisfies the $(O+)$ condition if for all $\mathrm{x}, \mathrm{y} \in \mathcal{D}, T(\mathrm{x}) \preceq_{n e} T(\mathrm{y}) \Leftrightarrow \mathrm{x} \preceq_{n e} \mathrm{y}$

(ii) Satisfies the $(O-)$ condition if for all $\mathrm{x}, \mathrm{y} \in \mathcal{D}, T(\mathrm{x}) \preceq_{n e} T(\mathrm{y}) \Leftrightarrow \mathrm{y} \preceq_{n e} \mathrm{x}$

Sufficient conditions for competitive maps to satisfy the $(O+)$ and $\left(\mathrm{O}_{-}\right)$conditions are provided in Theorem 4 [8].

Theorem 4 Consider $\mathcal{D} \subset \mathbb{R}^{2}$, where $\mathcal{D}$ is a Cartesian product of two intervals in $\mathbb{R}$. Let $T: \mathcal{D} \rightarrow \mathcal{D}$ be a competitive, $C^{(1)}$ map.

(i) If $T$ is injective and $\operatorname{det} J_{T}(\mathrm{x})>0$ for all $\mathrm{x} \in \mathcal{D}$ then $T$ satisfies $(O+)$ on $\mathcal{D}$.

(ii) If $T$ is injective and $\operatorname{det} J_{T}(\mathrm{x})<0$ for all $\mathrm{x} \in \mathcal{D}$ then $T$ satisfies $(O-)$ on $\mathcal{D}$.

Competitive maps that satisfy either of the conditions from Definition 4 exhibit well behaved global dynamics as evidenced by the following theorem, which was originally proven by deMottoni-Schiaffino and was later generalized for competitive maps by Smith [26].

Theorem 5 Suppose $\mathcal{D} \subset \mathbb{R}^{2}$. If $T$ is a competitive map that satisfies $(O+)$, then for all $\mathrm{x} \in \mathcal{D},\left\{T^{n}(\mathrm{x})\right\}$ is eventually component-wise monotone. If the orbit of $\mathrm{x}$ has compact closure, then it converges to a fixed point of $T$. If instead $\left(O_{-}\right)$is satisfied, then for all $\mathrm{x} \in \mathcal{D},\left\{T^{2 n}(\mathrm{x})\right\}$ is eventually component-wise monotone. If the orbit of $\mathrm{x}$ has compact closure in $\mathcal{D}$, then its omega limit set is either a period-two orbit or a fixed point.

The above results are utilized throughout Manuscripts 2 and 4, which deal specifically with monotone maps. 


\subsubsection{Global Manifolds}

Many authors have devoted time to developing general theory for determining the global dynamics for systems of difference equations. One important concept involves the existence of global stable and unstable manifolds. The local stable and unstable manifolds for a fixed point of the map (6) are defined in [23] as

$$
\begin{gathered}
\mathcal{W}_{l o c}^{s}(\bar{x}, \bar{y}):=\left\{(x, y): T^{n}(x, y) \rightarrow(\bar{x}, \bar{y}) \text { as } n \rightarrow \infty\right\} \\
\mathcal{W}_{l o c}^{u}(\bar{x}, \bar{y}):=\left\{(x, y): T^{-n}(x, y) \rightarrow(\bar{x}, \bar{y}) \text { as } n \rightarrow \infty\right\}
\end{gathered}
$$

Kulenović and Merino in $[11,27,28]$ present several theorems that allow the local manifolds to be extended in certain situations.

Theorem 6 Let $T$ be a competitive map on a rectangular region $\mathcal{D} \subset \mathbb{R}^{2}$. Let $\overline{\mathrm{x}} \in \mathcal{D}$ be a fixed point of $T$ such that $\Delta:=\mathcal{D} \cap \operatorname{int}\left(\mathcal{Q}_{1}(\overline{\mathrm{x}}) \cup \mathcal{Q}_{3}(\overline{\mathrm{x}})\right)$ is nonempty (i.e $\overline{\mathrm{x}}$ is not the $N W$ or $S E$ vertex of $\mathcal{D})$, and $T$ is strongly competitive on $\Delta$. Suppose that the following statements are true.

(a) The map $T$ has a $C^{(1)}$ extension to a neighborhood of $\overline{\mathrm{x}}$.

(b) The Jacobian $J_{T}(\overline{\mathrm{x}})$ of $T$ at $\overline{\mathrm{x}}$ has real eigenvalues $\lambda$, $\mu$ such that $0<|\lambda|<\mu$, where $|\lambda|<1$ and the eigenspace $E^{\lambda}$ associated with $\lambda$ is not a coordinate axis.

Then there exists a curve $\mathcal{C} \subset \mathcal{D}$ through $\overline{\mathrm{x}}$ that is invariant and a subset of the basin of attraction of $\overline{\mathrm{x}}$, such that $\mathcal{C}$ is tangential to the eigenspace $E^{\lambda}$ at $\overline{\mathrm{x}}$, and $\mathcal{C}$ is the graph of a strictly increasing continuous function of the first coordinate on an interval. Any endpoints of $\mathcal{C}$ in the interior of $\mathcal{D}$ are either fixed points or minimal period-two points. In the latter case, the set of endpoints of $\mathcal{C}$ is a minimal period-two orbit of $T$. 
In the particular systems that are studied in this thesis, the endpoints of $\mathcal{C}$ are boundary points of the region $\mathcal{D}$. This situation is further discussed in the following theorem from [28].

Theorem 7 For the curve $\mathcal{C}$ to have endpoints in $\partial \mathcal{D}$, it is sufficient that at least one of the following conditions is satisfied.

(i) The map T has no fixed points nor periodic points of minimal period-two in $\Delta$.

(ii) The map $T$ has no fixed points in $\Delta$, $\operatorname{det} \mathrm{J}_{\mathrm{T}}(\overline{\mathrm{x}})>0$, and $T(\overline{\mathrm{x}})=\overline{\mathrm{x}}$ has no solutions $\mathrm{x} \in \Delta$

(iii) The map $T$ has no points of minimal period-two in $\Delta$, $\operatorname{det} \mathrm{J}_{\mathrm{T}}(\overline{\mathrm{x}})>0$, and $T(\mathrm{x})=\overline{\mathrm{x}}$ has no solutions $\mathrm{x} \in \Delta$.

The existence of the curve $\mathcal{C}$ described in the previous two theorems is incredibly helpful in determining basins of attraction of fixed points. The following theorem expounds on this idea [28].

Theorem 8 Assume the hypotheses of Theorem 6, and let $\mathcal{C}$ be the curve whose existence is guaranteed by Theorem 6. If the endpoints of $\mathcal{C}$ belong to $\partial \mathcal{D}$, then $\mathcal{C}$ separates $\mathcal{D}$ into two connected components, namely

$$
\begin{aligned}
& \mathcal{W}_{-}:=\left\{\mathrm{x} \in \mathcal{D} \backslash \mathcal{C}: \exists \mathrm{y} \in \mathcal{C} \text { with } \mathrm{x} \preceq_{\text {se }} \mathrm{y}\right\} \\
& \mathcal{W}_{+}:=\left\{\mathrm{x} \in \mathcal{D} \backslash \mathcal{C}: \exists \mathrm{y} \in \mathcal{C} \text { with } \mathrm{y} \preceq_{\text {se }} \mathrm{x}\right\}
\end{aligned}
$$

such that the following statements are true

(i) $\mathcal{W}_{-}$is invariant and $\operatorname{dist}\left(\mathrm{T}^{\mathrm{n}}(\mathrm{x}), \mathcal{Q}_{2}(\overline{\mathrm{x}})\right) \rightarrow 0$ as $n \rightarrow \infty$ for every $\mathrm{x} \in \mathcal{W}_{-}$.

(ii) $\mathcal{W}_{+}$is invariant and $\operatorname{dist}\left(\mathrm{T}^{\mathrm{n}}(\mathrm{x}), \mathcal{Q}_{4}(\overline{\mathrm{x}})\right) \rightarrow 0$ as $n \rightarrow \infty$ for every $\mathrm{x} \in \mathcal{W}_{+}$. 
If, in addition $\overline{\mathrm{x}}$ is an interior point of $\mathcal{D}$ and $T$ is $C^{(2)}$ and strongly competitive in a neighborhood of $\overline{\mathrm{x}}$, then $T$ has no periodic points in the boundary of $\mathcal{Q}_{1}(\overline{\mathrm{x}}) \cup \mathcal{Q}_{3}(\overline{\mathrm{x}})$ except for $\overline{\mathrm{x}}$, and the following statements are true.

(i) For every $\mathrm{x} \in \mathcal{W}_{-}$there exists $n_{0} \in \mathbb{N}$ such that $T^{n}(\mathrm{x}) \in \operatorname{int} \mathcal{Q}_{2}(\overline{\mathrm{x}})$ for $n \geq n_{0}$.

(ii) For every $\mathrm{x} \in \mathcal{W}_{+}$there exists $n_{0} \in \mathbb{N}$ such that $T^{n}(\mathrm{x}) \in \operatorname{int} \mathcal{Q}_{4}(\overline{\mathrm{x}})$ for $n \geq n_{0}$.

The manuscripts of this thesis utilize the basic theory presented in the previous sections to establish global results for the population models introduced in Section 1.1 .

\section{List of References}

[1] J. G. Andrews and R. R. McLone, Mathematical Modeling. London: Butterworth-Heinwmann, 1976.

[2] J. M. Cushing, An Introduction to Structured Population Dynamics. Philadelphia: SIAM, 1998.

[3] L. J. S. Allen, An Introduction to Mathematical Biology. Upper Saddle River, New Jersey: Pearson Prentice Hall, 1987.

[4] F. Brauer and C. Castillo-Chávez, Mathematical Models in Population Biology and Epidemiology. New York: Springer-Verlag, 2001.

[5] Q. Din, "Global behavior of a host-parasitoid model under the constant refuge effect," Applied Mathematical Modeling, vol. 40, pp. 2815-2826, 2016.

[6] S. Ellner and J. Guckenheimer, Dynamic Models in Biology. Princeton, New Jersey: Princeton University Press, 2006.

[7] G. Livadiotis, L. Assas, B. Dennis, S. Elaydi, and E. Kwessi, "A discrete-time host-parasitoid model with an allee effect," Journal of Biological Dynamics, vol. 9, pp. 34-51, 2015.

[8] H. L. Smith, "Planar competitive and cooperative difference equations," Journal of Difference Equations and Applications, vol. 3, pp. 335-357, 1998.

[9] A. Bilgin, M. R. S. Kulenović, and E. Pilav, "Basins of attraction of period-two solutions of monotone systems of difference equations," Advances in Difference Equations, vol. 74, pp. 1-25, 2016. 
[10] A. Bilgin and M. R. S. Kulenović, "Two species competitive model with the allee effect," Advances in Difference Equations, vol. 307, pp. 1-27, 2014.

[11] M. R. S. Kulenović and O. Merino, "Competitive-exclusion versus competitive-coexistence for systems in the plane," Discrete Contin. Dyn. Syst. Ser. B, vol. 6, pp. 1141-1156, 2006.

[12] J. Cushing, S. Levarge, N. Chitnis, , and S. Henson, "Some discrete competition models and the competitive exclusion principle," Journal of Difference Equations and Applications, vol. 10, pp. 1139-1151, 2004.

[13] P. Leslie and J. Gower, "The properties of a stochastic model for two competing species," Biometrika, vol. 45, pp. 316-330, 1958.

[14] T. Park, "Experimental studies of inter-species competition: competition between populations of the flour beetles tribolium confusum duval and tribolium castaneum herbst," Ecological Monograph, vol. 18, pp. 265-308, 1948.

[15] M. Gidea, J. D. Meiss, I. Ugarcovici, and H. Weiss, "Applications of kam thoery to population dynamics," Journal of Biological Dynamics, vol. 1, pp. 1-23, 2009.

[16] M. Hassell, The Spatial and Temporal Dynamics of Host-Parasitoid Interactions. New York: Oxford University Press, 2000.

[17] A. Khan and M. Qureshi, "Dynamics of a modified nicholson-bailey hostparasitoid model," Advances in Difference Equations, vol. 23, pp. 1-15, 2015.

[18] Q. Din and T. Donchev, "Global character of a host-parasite model," Chaos, Solitons 6 Fractals, vol. 54, pp. 1-7, 2013.

[19] P. H. Leslie, "Some further notes on the use of matrices in population mathematics," Biometrika, vol. 55, pp. 213-245, 1948.

[20] S. Friedland and S. Karlin, "Some inequalities for the spectral radius of nonnegative matrices and applications," Duke Mathematical Journal, vol. 42, pp. 459-489, 1975.

[21] S. Townley, R. Rebarber, and B. Tenhumberg, "Feedback control systems analysis of density dependent population dynamics," Systems and Control Letters, vol. 61, pp. 309-315, 2012.

[22] S. Elaydi, An Introduction to Difference Equations, 3rd Ed. New York: Springer-Verlage, 2005.

[23] M. R. S. Kulenović and O. Merino, Discrete Dynamical Systems and Difference Equations with Mathematica. Boca Raton: Chapman\& Hall/CRC Press, 2002. 
[24] S. Sternberg, Dynamical Systems. Dover Publications, 2013.

[25] E. N. Dancer and P. Hess, "Stability of fixed points for order-preserving discrete-time dynamical systems," J. reine angew. Math., vol. 419, pp. 125139, 1991.

[26] H. L. Smith, "Periodic competitive differential equations and the discrete dynamics of competitive maps," Journal of Differential Equations, vol. 64, pp. 165-194, 1986.

[27] M. R. S. Kulenović and O. Merino, "Global bifurcation for competitive systems in the plane," Discrete Contin. Dyn. Syst. Ser. B, vol. 12, pp. 133-149, 2009.

[28] M. R. S. Kulenović and O. Merino, "Invariant manifolds for competitive discrete systems in the plane," Int. J. of Bifurcations and Chaos, vol. 20, pp. 2471-2486, 2010. 


\title{
MANUSCRIPT 2 \\ Global Dynamics of Leslie-Gower Competitive Systems in the Plane
}

\author{
M.R.S Kulenović and David T. McArdle
}

Publication Status:

Submitted March 7th, 2017, Advances in Difference Equations

Keywords: Bifurcation, competitive, map, global stable manifold, monotonicity, period-2 solution

AMS Subject Classification: Primary: 37G35, Secondary: 39A10, 39A20 


\section{Abstract}

Global dynamic results are obtained for families of competitive systems of difference equations of the form

$$
x_{n+1}=\frac{b_{1} x_{n}}{\alpha_{1}+x_{n}+c_{1} y_{n}}, \quad y_{n+1}=\frac{b_{2} y_{n}}{\alpha_{2}+c_{2} x_{n}+y_{n}} \quad n=0,1, \ldots
$$

where the parameters $b_{1}, b_{2}$ are positive real numbers, and $\alpha_{1}, \alpha_{2}, c_{1}$, and $c_{2}$ and the initial conditions $x_{0}$ and $y_{0}$ are arbitrary nonnegative numbers. In particular, we investigate the effect of parameters $\alpha_{1}, \alpha_{2}$ on the global dynamics of this system.

\section{$2.1 \quad$ Introduction}

Consider the system of difference equations

$$
x_{n+1}=\frac{b_{1} x_{n}}{\alpha_{1}+x_{n}+c_{1} y_{n}}, \quad y_{n+1}=\frac{b_{2} y_{n}}{\alpha_{2}+c_{2} x_{n}+y_{n}} \quad n=0,1, \ldots
$$

where the parameters $b_{1}, b_{2}$ are positive real numbers, and $\alpha_{1}, \alpha_{2}, c_{1}$, and $c_{2}$ and the initial conditions $x_{0}$ and $y_{0}$ are arbitrary nonnegative numbers. We consider the effect of terms $\alpha_{1}, \alpha_{2}$ on the global dynamics of system (1). The global dynamics of (1) was considered in the case where the parameters $\alpha_{1}, \alpha_{2}$ are positive in $[1,2]$ and the complete description of the dynamics was given in [2], where the following result was obtained:

Assuming, without loss of generality, that $\alpha_{1}=\alpha_{2}=1$, it has been shown in [1] that under the condition $b_{1}>1$ and $b_{2}>1$, the points

$$
E_{0}(0,0), \quad E_{1}\left(b_{1}-1,0\right), \quad E_{2}\left(0, b_{2}-1\right)
$$

are equilibria of equation (1), and that for some values of the parameters there exists an additional equilibrium point $E_{3}$, located in the open positive quadrant, given by

$$
E_{3}\left(\frac{b_{2}-1}{c_{1} c_{2}-1}\left(c_{1}-\frac{b_{1}-1}{b_{2}-1}\right), \frac{b_{1}-1}{c_{1} c_{2}-1}\left(c_{2}-\frac{b_{2}-1}{b_{1}-1}\right)\right) .
$$


Important subsets of parameter space are described in Table 1, together with corresponding behavior of fixed points established in [1].

\begin{tabular}{|c|c|c|}
\hline Condition & $c_{1}\left(b_{2}-1\right)<b_{1}-1$ & $c_{1}\left(b_{2}-1\right)>b_{1}-1$ \\
\hline$c_{2}\left(b_{1}-1\right)<b_{2}-1$ & $\begin{array}{l}\text { Case } \mathbf{1 .} \\
E_{0} \text { is a repeller } \\
E_{1} \text { is a saddle } \\
E_{2} \text { is a saddle } \\
E_{3} \text { is interior local attractor }\end{array}$ & $\begin{array}{l}\text { Case } 2 \text {. } \\
E_{0} \text { is a repeller } \\
E_{1} \text { is a saddle } \\
E_{2} \text { attractor on }[0, \infty) \times(0, \infty) \\
\text { No interior fixed point exists }\end{array}$ \\
\hline$c_{2}\left(b_{1}-1\right)>b_{2}-1$ & $\begin{array}{l}\text { Case } 3 \text {. } \\
E_{0} \text { is a repeller } \\
E_{1} \text { attractor on }(0, \infty) \times[0, \infty) \\
E_{2} \text { is a saddle } \\
\text { No interior fixed point exists }\end{array}$ & $\begin{array}{l}\text { Case } 4 . \\
E_{0} \text { is a repeller } \\
E_{1} \text { is a local attractor } \\
E_{2} \text { is a local attractor } \\
E_{3} \text { is an interior saddle }\end{array}$ \\
\hline
\end{tabular}

Table 1: Global behavior of solutions to (1) when $b_{1}>1$ and $b_{2}>1$. Equality relations are not represented for the sake of a simpler description.

An application of Theorem 9 in [2] applies when parameters vary from Case 2 to Case 4 of Table 1. Set

$$
\mathcal{A}=\left\{\alpha \in \mathbb{R}_{+}^{4}: \alpha=\left(b_{1}, b_{2}, c_{1}, c_{2}\right) \text { and } c_{1}\left(b_{2}-1\right)>b_{1}-1>0\right\}
$$

and define $T_{\alpha}$ to be the map of system (1) restricted to $\mathcal{R}=[0, \infty) \times(0, \infty)$, that is,

$$
T_{\alpha}(x, y)=\left(\frac{b_{1} x}{1+x+c_{1} y}, \frac{b_{2} y}{1+c_{2} x+y}\right) .
$$

Therefore, Theorem 9 in [2] gives global behavior of solutions to system (1) on $\mathcal{R}=[0, \infty) \times(0, \infty)$ for $\alpha \in \mathcal{A}$. In particular, a bifurcation occurs when the equilibrium $\overline{\mathrm{x}}_{\alpha}$ changes its local character from a locally stable equilibrium to a saddle point. This happens when the parameters cross the critical surface $\Gamma\left(b_{1}, b_{2}, c_{1}, c_{2}\right)=c_{2}\left(b_{1}-1\right)-b_{2}+1=0$.

It is also shown in [1] that the open, positive semiaxis $(0, \infty) \times\{0\}$ is attracted to $E_{1}$, and that the open, positive semiaxis $\{0\} \times(0, \infty)$ is attracted to $E_{2}$. The 
following two results describe the global dynamics of system (1) in all cases. The first result gives the global dynamics in the hyperbolic case and the second result in the non-hyperbolic case.

Theorem 1 Consider system (1).

(i) Suppose that $c_{1}\left(b_{2}-1\right)>b_{1}-1>0$. If $c_{2}\left(b_{1}-1\right)>b_{2}-1$, then $E_{2}$ is globally asymptotically stable on $[0, \infty) \times(0, \infty)$ and $E_{1}$ attracts all points on the open semiaxis $(0, \infty) \times\{0\}$. If $c_{2}\left(b_{1}-1\right)<b_{2}-1$, then the stable manifold $\mathcal{W}^{s}\left(E_{3}\right)$ in $[0, \infty) \times[0, \infty)$ is the graph of a continuous, increasing function of the first coordinate. Furthermore, a solution $\left\{\mathrm{x}_{n}\right\}$ converges to $E_{1}$ whenever $\mathrm{x}_{0}$ is below $\mathcal{W}^{s}\left(E_{3}\right)$, and $\left\{\mathrm{x}_{n}\right\}$ converges to $E_{2}$ whenever $\mathrm{x}_{0}$ is above $\mathcal{W}^{s}\left(E_{3}\right)$.

(ii) Suppose that $c_{1}\left(b_{2}-1\right)<b_{1}-1>0$. If $c_{2}\left(b_{1}-1\right)>b_{2}-1$, then $E_{1}$ is globally asymptotically stable on $(0, \infty) \times[0, \infty)$ and $E_{2}$ attracts all points on the open semiaxis $\{0\} \times(0, \infty)$. If $c_{2}\left(b_{1}-1\right)<b_{2}-1$, then $E_{3}$ is globally asymptotically stable on $(0, \infty) \times(0, \infty), E_{1}$ attracts all points on the open semiaxis $(0, \infty) \times\{0\}$, and $E_{2}$ attracts all points on the open semiaxis $\{0\} \times$ $(0, \infty)$

See Figure 1 for graphical interpretation.

The non-hyperbolic case when

$$
c_{1}\left(b_{2}-1\right)=b_{1}-1 \quad \text { and } \quad c_{2}\left(b_{1}-1\right)=b_{2}-1
$$

was not considered in [1]. When (3) holds, a direct calculation gives that the equilibrium points of $T$ are $E_{0}(0,0)$ and all points on the segment $\mathcal{E}:=\left\{E_{t}: 0 \leq\right.$ $t \leq 1\}$, where

$$
E_{t}:=\left(\left(b_{1}-1\right)(1-t),\left(b_{2}-1\right) t\right), \quad 0 \leq t \leq 1
$$


The eigenvalues of the Jacobian of $T$ at $E_{t}$ are

$$
\lambda_{1}=1 \quad \text { and } \quad \lambda_{2}=(1-t) \frac{1}{b_{1}}+t \frac{1}{b_{2}}, \quad 0 \leq t \leq 1
$$

and the corresponding eigenvectors are

$$
\mathrm{e}_{1}=\left(-\frac{1-b_{1}}{1-b_{2}}, 1\right) \text { and } \mathrm{e}_{2}=\left(b_{2}\left(1-b_{1}\right)^{2}(1-t), b_{1}\left(1-b_{2}\right)^{2} t\right), \quad 0 \leq t \leq 1
$$

It is shown in [3] that, for system (1), the hypotheses of Theorem 5 in [4] are satisfied and that all solutions fall inside an invariant rectangular region. Therefore, every solution of (1) converges to an equilibrium point. A direct calculation shows that the origin is a repeller. We conclude that every nonzero solution converges to a point $(\bar{x}, \bar{y}) \in \mathcal{E}$. Also, with an argument similar to the one used in [5], one has that the equilibrium depends continuously on the initial condition. That is, if $T^{*}(x, y):=\lim T^{n}(x, y)$, then $T^{*}$ is continuous. These observations, together with an application of Theorem 1 in [4] lead to the following result.

Theorem 2 Assume (3) holds. Then,

(i) Every nonzero solution to system (1) converges to an equlibrium $(\bar{x}, \bar{y}) \in \mathcal{E}$.

(ii) For every $(\bar{x}, \bar{y}) \in \mathcal{E}$ with $\bar{x} \neq 0$ and $\bar{y} \neq 0$, the stable set $\mathcal{W}_{(\bar{x}, \bar{y})}^{s}$ is an unbounded, increasing curve $\mathcal{C}$ with endpoint $(0,0)$.

(iii) The limiting equilibrium varies continuously with the initial condition.

See Figure 1 for graphical interpretation.

Statement (ii) excludes equilibria of the form $(0, \bar{y})$ and $(\bar{x}, 0)$ since the hypotheses of Theorem 1 in [4] are not satisfied at these points.

In this paper we consider two related systems, namely

$$
x_{n+1}=\frac{b_{1} x_{n}}{x_{n}+c_{1} y_{n}}, \quad y_{n+1}=\frac{b_{2} y_{n}}{c_{2} x_{n}+y_{n}} \quad n=0,1, \ldots
$$




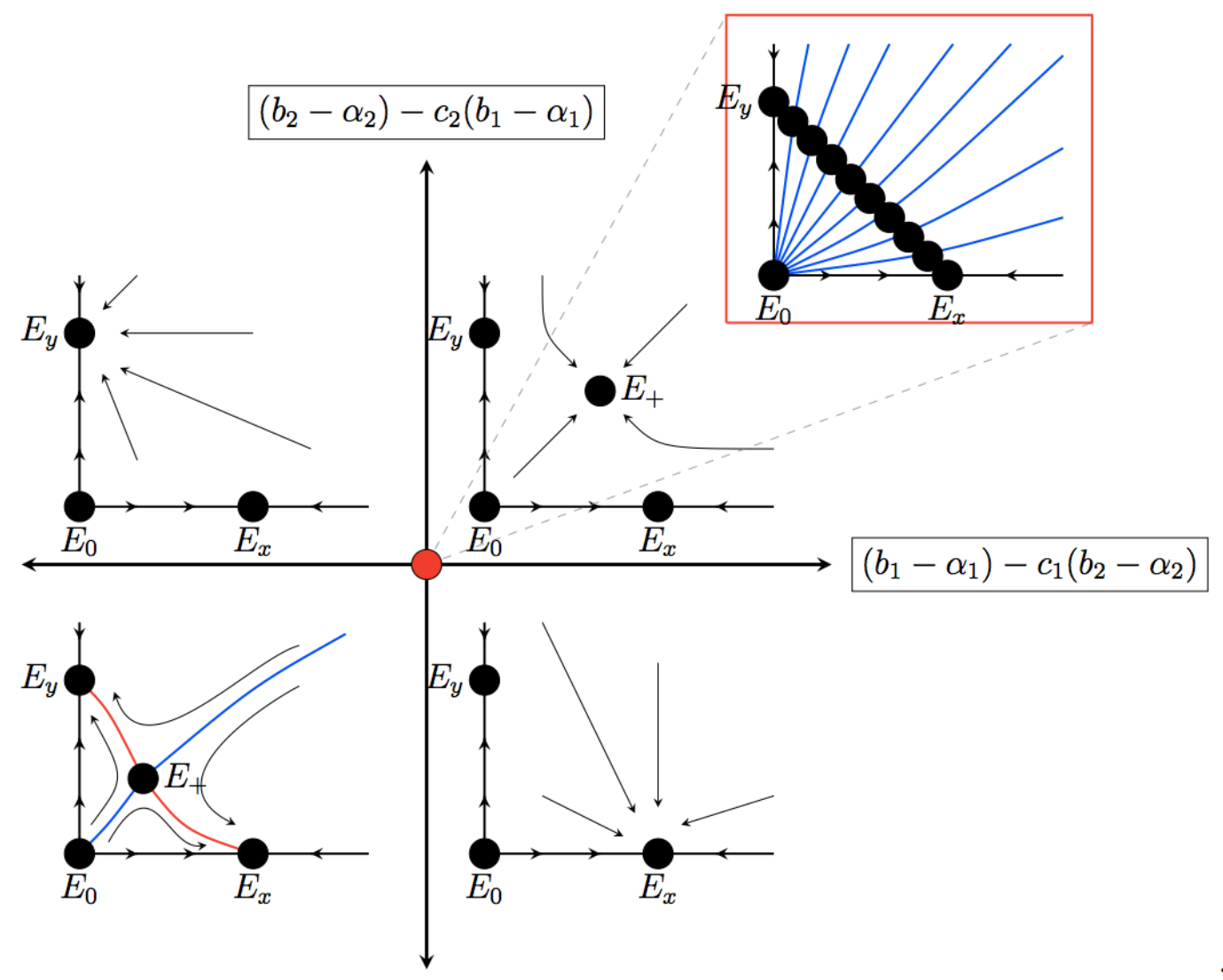

Figure 1: Global dynamics of system (1).

and

$$
x_{n+1}=\frac{b_{1} x_{n}}{\alpha_{1}+x_{n}+c_{1} y_{n}}, \quad y_{n+1}=\frac{b_{2} y_{n}}{c_{2} x_{n}+y_{n}} \quad n=0,1, \ldots,
$$

where all present coefficients are positive and the initial conditions are nonnegative. We derive the global dynamics of both systems (4) and (5), which explains the effect of the parameters $\alpha_{1}, \alpha_{2}$ on the global dynamics.

The paper is organized as follows. The second section presents some basic preliminary results about competitive systems, which is our main tool in proving the results. The third section contains the global dynamics of system (4) and the fourth section gives the global dynamics of system (5). The fifth section presents some results on global dynamic scenarios for general competitive systems. Actually, we show that all global dynamic results that hold for any of the three systems (1), 
(4), and (5) can be immediately applied to a general competitive system and that the global behavior of all three systems is determined by the linearized dynamics. We will compare and contrast the global dynamics of (4) and (5) with that of system (1).

\subsection{Preliminaries}

In this section we provide some basic facts about competitive maps and systems of difference equations in the plane.

Definition 5 Let $\mathcal{R}$ be a subset of $\mathbb{R}^{2}$ with nonempty interior, and let $T: \mathcal{R} \rightarrow \mathcal{R}$ be a map (i.e. a continuous function). Set $T(x, y)=(f(x, y), g(x, y))$. The map $T$ is competitive if $f(x, y)$ is nondecreasing in $x$ and nonincreasing in $y$ and $g(x, y)$ is nonincreasing in $x$ and nondecreasing in $y$. If both $f$ and $g$ are nondecreasing in $x$ and $y$, we say that $T$ is cooperative. If $T$ is competitive (cooperative), the associated system of difference equations

$$
\left\{\begin{array}{l}
x_{n+1}=f\left(x_{n}, y_{n}\right) \\
y_{n+1}=g\left(x_{n}, y_{n}\right)
\end{array}, \quad n=0,1,2, \ldots, \quad\left(x_{0}, y_{0}\right) \in R\right.
$$

is said to be competitive (cooperative). The map $T$ and the associated system of difference equations are said to be strongly competitive (strongly cooperative) if the adjectives nondecreasing and nonincreasing are replaced by increasing and decreasing.

If $T$ is differentiable, a sufficient condition for $T$ to be strongly competitive is that the Jacobian matrix of $T$ at any point $(x, y) \in \mathcal{R}$ has the sign configuration

$$
\left(\begin{array}{cc}
+ & - \\
- & +
\end{array}\right)
$$

Competitive systems of the form (6) have been studied by many authors such as Clark, Kulenović, and Selgrade, Hess, Hirsch and Smith, Kulenović, Merino, and 
Nurkanović, Leonard and May, Smale and others [6, 7, 8, 9, 10, 11, 12, 13]. See [14] for interesting applications of this theory to basic models in population dynamics.

Denote with $\preceq_{\text {se }}$ the South-East partial order in the plane whose nonnegative cone is the standard fourth quadrant $\{(x, y): x \geq 0, y \leq 0\}$, that is, $\left(x_{1}, y_{1}\right) \preceq$ $\left(x_{2}, y_{2}\right)$ if and only if $x_{1} \leq x_{2}$ and $y_{1} \geq y_{2}$. The North-East partial order $\preceq_{n e}$ is defined analogously with the nonnegative cone given by the standard first quadrant $\{(x, y): x \geq 0, y \geq 0\}$.

Competitive maps $T$ in the plane preserve the South-East ordering: $T(\mathrm{u}) \preceq_{s e}$ $T(\mathrm{v})$ whenever $\mathrm{u} \preceq_{s e} \mathrm{v}$. Similarly, cooperative maps in the plane preserve the North-East ordering. In fact, the concepts of competitive and cooperative (for maps) may be defined in terms of the order preserving properties of maps. Thus the theory of competitive maps is a special case of the theory of order preserving maps (or monotone operators).

Order preserving maps in $\mathbb{R}^{n}$, and in particular competitive maps in $\mathbb{R}^{2}$, may have chaotic dynamics. Smale [12] showed that any continuous time vector field on the standard $(n-1)$-simplex in $\mathbb{R}^{n}$ can be embedded on a smooth, competitive vector field in $\mathbb{R}^{n}$ for which the simplex is an attractor. In the case of a planar system (6), this means that any first order difference equation, including chaotic, can be embedded into a competitive system (6) in the plane. An effective algebraic method to do this is provided by Smith in [13].

Let $\preceq$ be a partial order on $\mathbb{R}^{n}$ with nonnegative cone $\mathcal{P}$. For $\mathrm{x}, \mathrm{y} \in \mathbb{R}^{n}$ the order interval $\llbracket \mathrm{x}, \mathrm{y} \rrbracket$ is the set of all $\mathrm{z}$ such that $\mathrm{x} \preceq \mathrm{z} \preceq \mathrm{y}$. We say $\mathrm{x} \prec \mathrm{y}$ if $\mathrm{x} \preceq \mathrm{y}$ and $\mathrm{x} \neq \mathrm{y}$. Also, $\mathrm{x} \ll \mathrm{y}$ if $\mathrm{y}-\mathrm{x} \in \operatorname{int}(\mathcal{P})$. A map $T$ on a subset of $\mathbb{R}^{n}$ is order preserving if $T(\mathrm{x}) \preceq T(\mathrm{y})$ whenever $\mathrm{x} \preceq \mathrm{y}$, strictly order preserving if $T(\mathrm{x}) \prec T(\mathrm{y})$ whenever $\mathrm{x} \prec \mathrm{y}$, and strongly order preserving if $T(\mathrm{x}) \ll T(\mathrm{y})$ whenever $\mathrm{x} \prec \mathrm{y}$. 
Let $T: \mathcal{R} \rightarrow \mathcal{R}$ be a map with a fixed point $\overline{\mathrm{x}}$ and let $\mathcal{R}^{\prime}$ be an invariant subset of $\mathcal{R}$ that contains $\overline{\mathrm{x}}$. We say that $\overline{\mathrm{x}}$ is stable (asymptotically stable) relative to $\mathcal{R}^{\prime}$ if $\overline{\mathrm{x}}$ is a stable (asymptotically stable) fixed point of the restriction of $T$ to $\mathcal{R}^{\prime}$.

Definition 6 Let $\mathcal{R}$ be a nonempty subset of $\mathbb{R}^{2}$. A competitive map $T: \mathcal{R} \rightarrow \mathcal{R}$ is said to satisfy condition $(O+)$ if for every $\mathrm{x}, \mathrm{y}$ in $\mathcal{R}, T(\mathrm{x}) \preceq_{n e} T(\mathrm{y})$ implies $\mathrm{x} \preceq_{n e} \mathrm{y}$, and $T$ is said to satisfy condition $(O-)$ if for every $\mathrm{x}$, $\mathrm{y}$ in $\mathcal{R}, T(\mathrm{x}) \preceq_{n e}$ $T(\mathrm{y})$ implies $\mathrm{y} \preceq_{n e} \mathrm{x}$.

The following theorem was proved by deMottoni-Schiaffino for the Poincaré map of a periodic competitive Lotka-Volterra system of differential equations. Smith generalized the proof to competitive and cooperative maps [15].

Theorem 3 Let $\mathcal{R}$ be a nonempty subset of $\mathbb{R}^{2}$. If $T$ is a competitive map for which $(O+)$ holds, then for all $\mathrm{x} \in \mathcal{R},\left\{T^{n}(\mathrm{x})\right\}$ is eventually componentwise monotone. If the orbit of $\mathrm{x}$ has compact closure, then it converges to a fixed point of $T$. If instead $\left(\mathrm{O}_{-}\right)$holds, then for all $\mathrm{x} \in \mathcal{R},\left\{T^{2 n}(\mathrm{x})\right\}$ is eventually componentwise monotone. If the orbit of $\mathrm{x}$ has compact closure in $\mathcal{R}$, then its omega limit set is either a period-two orbit or a fixed point.

The next two results are stated for order-preserving maps on $\mathbb{R}^{n}$. These results are known but are given here for completeness. See [7] for a more general version that is valid in ordered Banach spaces.

Theorem 4 For a nonempty set $\mathcal{R} \subset \mathbb{R}^{n}$ and $\preceq$ a partial order on $\mathbb{R}^{n}$, let $T$ : $\mathcal{R} \rightarrow \mathcal{R}$ be an order preserving map, and let $\mathrm{a}, \mathrm{b} \in \mathcal{R}$ be such that $\mathrm{a} \prec \mathrm{b}$ and $\llbracket \mathrm{a}, \mathrm{b} \rrbracket \subset \mathcal{R}$. If $\mathrm{a} \preceq T(\mathrm{a})$ and $T(\mathrm{~b}) \preceq \mathrm{b}$, then $\llbracket \mathrm{a}, \mathrm{b} \rrbracket$ is invariant and

i. There exists a fixed point of $T$ in $\llbracket a, b \rrbracket$. 
ii. If $T$ is strongly order preserving, then there exists a fixed point in $\llbracket \mathrm{a}, \mathrm{b} \rrbracket$ which is stable relative to $\llbracket \mathrm{a}, \mathrm{b} \rrbracket$.

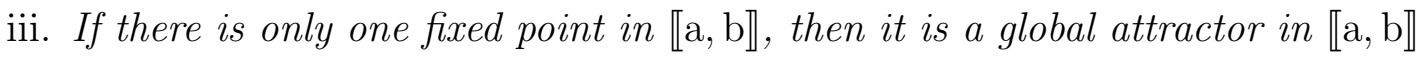
and therefore asymptotically stable relative to $\llbracket \mathrm{a}, \mathrm{b} \rrbracket$.

Corollary 2 If the nonnegative cone of $\preceq$ is a generalized quadrant in $\mathbb{R}^{n}$, and if $T$ has no fixed points in $\llbracket \mathrm{u}_{1}, \mathrm{u}_{2} \rrbracket$ other than $\mathrm{u}_{1}$ and $\mathrm{u}_{2}$, then the interior of $\llbracket \mathrm{u}_{1}, \mathrm{u}_{2} \rrbracket$ is either a subset of the basin of attraction of $\mathrm{u}_{1}$ or a subset of the basin of attraction of $\mathrm{u}_{2}$.

Our main tool will be results from $[2,4,16]$ regarding the existence of the global stable and unstable manifolds of competitive maps in the plane.

The non-hyperbolic equilibrium solution of system (6) is said to be of stable (resp. unstable) type if the second eigenvalue of the Jacobian matrix evaluated at the equilibrium solution is by absolute value less than 1 (resp. bigger than 1 ).

\subsection{Global Dynamics of System (4)}

First we give some basic results about the global behavior of system (4). Denote by

$$
T(x, y)=\left(\frac{b_{1} x}{x+c_{1} y}, \frac{b_{2} y}{c_{2} x+y}\right),
$$

the map associated with system (4). System (4) is homogeneous and was partially investigated in [17].

Lemma 1 The following statements hold:

(a) Every solution of system (4) satisfies $x_{n} \leq b_{1}, y_{n} \leq b_{2}, \quad n \geq 1$.

(b) $\operatorname{det} J_{T}(x, y)=0$ for every $(x, y)$, where $J_{T}$ denotes the Jacobian matrix of the map $T$. 
(c) $T\left(x_{0}, 0\right)=E_{x}\left(b_{1}, 0\right), T\left(0, y_{0}\right)=E_{y}\left(0, b_{2}\right)$ for every $x_{0}>0, y_{0}>0$.

(d) Every solution of system (4) satisfies the difference equation

$$
r_{n+1}=\frac{b_{2}}{b_{1}} r_{n} \frac{1+c_{1} r_{n}}{c_{2}+r_{n}}, \quad n \geq 0
$$

where $r_{n}=y_{n} / x_{n}$.

(e) If

$$
\left(b_{2}-b_{1} c_{2}\right)\left(b_{1}-b_{2} c_{1}\right)>0
$$

then the map $T$ has an invariant line

$$
\ell_{S}: y=\frac{b_{2}-b_{1} c_{2}}{b_{1}-b_{2} c_{1}} x
$$

Proof. The Jacobian matrix $J_{T}$ of the map $T$ has the form

$$
J_{T}(x, y)=\left(\begin{array}{cc}
\frac{b_{1} c_{1} y}{\left(x+c_{1} y\right)^{2}} & -\frac{b_{1} c_{1} x}{\left(x+c_{1} y\right)^{2}} \\
-\frac{b_{2} c_{2} y}{\left(c_{2} x+y\right)^{2}} & \frac{b_{2} c_{2} x}{\left(c_{2} x+y\right)^{2}}
\end{array}\right),
$$

which implies (b). Parts (a) and (c) follow by immediate checking. Part (d) follows by dividing equations of system (4). Part (e) follows from (d) since $\frac{b_{2}-b_{1} c_{2}}{b_{1}-b_{2} c_{1}}$ is exactly an equilibrium of the equation in $(d)$.

System (4) always has two equilibrium solutions on the axes, $E_{x}\left(b_{1}, 0\right)$, $E_{y}\left(0, b_{2}\right)$. It can also have either exactly one interior equilibrium solution $E$ or an infinite number of interior equilibrium solutions $E_{t}$. Since the interior equilibrium solution $E$ is an intersection of two equilibrium curves, $\mathcal{C}_{1}: x+c_{1} y=b_{1}$ and $\mathcal{C}_{2}: c_{2} x+y=b_{2}$, it will exist if either $\frac{b_{2}}{c_{2}}<b_{1}, \frac{b_{1}}{c_{1}}<b_{2}$ (x-intercept of $\mathcal{C}_{2}$ smaller than $x$-intercept of $\mathcal{C}_{1}$ and $y$-intercept of $\mathcal{C}_{2}$ bigger than $y$-intercept of $\mathcal{C}_{1}$ ) or $\frac{b_{2}}{c_{2}}>b_{1}, \frac{b_{1}}{c_{1}}>b_{2}\left(x\right.$-intercept of $\mathcal{C}_{1}$ smaller than $x$-intercept of $\mathcal{C}_{2}$ and $y$-intercept of $\mathcal{C}_{1}$ bigger than $y$-intercept of $\mathcal{C}_{2}$ ). These two geometrical conditions can be unified as condition (7). 


\begin{tabular}{|c|c|}
\hline Condition & Equilibrium Points \\
\hline \hline$\left(b_{2}-b_{1} c_{2}\right)\left(b_{1}-b_{2} c_{1}\right)<0$, & \\
$b_{2}=b_{1} c_{2}$ and $b_{1} \neq b_{2} c_{1}$, & $E_{x}, E_{y}$ \\
or $b_{2} \neq b_{1} c_{2}$ and $b_{1}=b_{2} c_{1}$ & \\
\hline$\left(b_{2}-b_{1} c_{2}\right)\left(b_{1}-b_{2} c_{1}\right)>0$ & $E_{x}, E_{y}, E$ \\
\hline$b_{2}=b_{1} c_{2}$ and $b_{1}=b_{2} c_{1}$ & $E_{x}, E_{y}, E_{t}$ \\
\hline
\end{tabular}

Table 2: The equilibrium points for system (4).

Condition (7) implies that $c_{1} c_{2} \neq 1$, in which case the interior equilibrium $E(\bar{x}, \bar{y})$ is given as:

$$
\bar{x}=\frac{b_{1}-b_{2} c_{1}}{1-c_{1} c_{2}}, \quad \bar{y}=\frac{b_{2}-b_{1} c_{2}}{1-c_{1} c_{2}} .
$$

Notice that $b_{2}<b_{1} c_{2}, b_{1}<b_{2} c_{1}$ implies that $c_{1} c_{2}>1$ and $b_{2}>b_{1} c_{2}, b_{1}>b_{2} c_{1}$ implies that $c_{1} c_{2}<1$. If either $\left(b_{2}-b_{1} c_{2}\right)\left(b_{1}-b_{2} c_{1}\right)<0, b_{2}=b_{1} c_{2}$ and $b_{1} \neq$ $b_{2} c_{1}$, or $b_{1}=b_{2} c_{1}$ and $b_{2} \neq b_{1} c_{2}$, then there are no interior equilibrium points. Furthermore, if $b_{2}=b_{1} c_{2}$ and $b_{1}=b_{2} c_{1}$, the two equilibrium curves $\mathcal{C}_{1}, \mathcal{C}_{2}$ coincide and every point on the segment $x+c_{1} y=b_{1}, x, y \geq 0$ is an equilibrium solution $E_{t}\left(b_{1}-c_{1} t, t\right), t \in\left[0, b_{2}\right]$. See Table 2 for a summary of the equilibrium points of system (4).

The following result describes the local stability character of all equilibrium solutions.

Lemma 2 Consider system (4).

(a) The equilibrium solution $E_{x}$ is locally asymptotically stable if $b_{2}<b_{1} c_{2}$, nonhyperbolic of stable type if $b_{2}=b_{1} c_{2}$, and a saddle point if $b_{2}>b_{1} c_{2}$. In each case, the eigenvectors associated with the eigenvalues $\lambda_{1}=0$ and $\lambda_{2}=\frac{b_{2}}{b_{1} c_{2}}$ are $\mathrm{e}_{1}=(1,0)$ and $\mathrm{e}_{2}=\left(-\frac{b_{1} c_{1} c_{2}}{b_{2}}, 1\right)$.

(b) The equilibrium solution $E_{y}$ is locally asymptotically stable if $b_{1}<b_{2} c_{1}$, nonhyperbolic of stable type if $b_{1}=b_{2} c_{1}$, and a saddle point if $b_{1}>b_{2} c_{1}$. In each 
case, the eigenvectors associated with the eigenvalues $\lambda_{1}=0$ and $\lambda_{2}=\frac{b_{1}}{b_{2} c_{1}}$ are $\mathrm{e}_{1}=(0,1)$ and $\mathrm{e}_{2}=\left(\frac{b_{1}}{c_{1} c_{2} b_{2}},-1\right)$.

(c) The interior equilibrium solution $E$ is a saddle point when $b_{2}<b_{1} c_{2}$ and $b_{1}<b_{2} c_{1}$ and is locally asymptotically stable when $b_{2}>b_{1} c_{2}$ and $b_{1}>b_{2} c_{1}$.

(d) The interior equilibrium solutions $E_{t}$ are non-hyperbolic of the stable type and the eigenvector which corresponds to $\lambda_{1}=0$ is given as $\mathrm{e}_{1}=(1, y)$.

Proof.

(a) In view of (9), we have

$$
J_{T}\left(E_{x}\right)=\left(\begin{array}{cc}
0 & -c_{1} \\
0 & \frac{b_{2}}{b_{1} c_{2}}
\end{array}\right),
$$

which implies that the eigenvalues of the Jacobian matrix are $\lambda_{1}=0, \lambda_{2}=$ $\frac{b_{2}}{b_{1} c_{2}}$. The corresponding eigenvectors are as stated.

(b) In view of (9), we have

$$
J_{T}\left(E_{y}\right)=\left(\begin{array}{cc}
\frac{b_{1}}{b_{2} c_{1}} & 0 \\
-c_{2} & 0
\end{array}\right)
$$

which implies that the eigenvalues of the Jacobian matrix are $\lambda_{1}=0, \lambda_{2}=$ $\frac{b_{1}}{b_{2} c_{1}}$. The corresponding eigenvectors are as stated.

(c) The eigenvalues of the Jacobian matrix evaluated at the equilibrium $E$, $\lambda_{1}=0$ and $\lambda_{2}=\operatorname{tr}\left(J_{T}(E)\right)$, correspond to the roots of the characteristic polynomial $p(t)=t^{2}-\operatorname{tr}\left(J_{T}(E)\right) t$. Note that $\lambda_{2}>0$ by (9). Furthermore

$$
\begin{gathered}
p(1)=\frac{\left(b_{1}-b_{2} c_{1}\right)\left(b_{2}-b_{1} c_{2}\right)}{b_{1} b_{2}\left(1-c_{1} c_{2}\right)}, \\
p^{\prime}(1)=\frac{b_{2}\left(b_{1}-b_{2} c_{1}\right)+b_{1}\left(b_{2}-b_{1} c_{2}\right)}{b_{1} b_{2}\left(1-c_{1} c_{2}\right)} .
\end{gathered}
$$


Consequently, if $b_{2}<b_{1} c_{2}$ and $b_{1}<b_{2} c_{1}$, then $p(1)<0$ and $p^{\prime}(1)>0$ and if $b_{2}>b_{1} c_{2}$ and $b_{1}>b_{2} c_{1}$, then $p(1)>0$ and $p^{\prime}(1)>0$. It follows that $E$ is a saddle point when $b_{2}<b_{1} c_{2}$ and $b_{1}<b_{2} c_{1}$ and is locally asymptotically stable when $b_{2}>b_{1} c_{2}$ and $b_{1}>b_{2} c_{1}$.

(d) In this case, the eigenvalues of the Jacobian matrix evaluated at the equilibrium $E_{t}$ are $\lambda_{1}=0, \lambda_{2}=1$. The eigenvector that corresponds to $\lambda_{1}=0$ is $\mathrm{e}_{1}=(1, y)$, where $y>0$ satisfies $x+c_{1} y=b_{1}$ and points towards the first quadrant.

Now, global behavior of system (4) is described by the following result:

Theorem 5 Consider system (4).

(a) If $b_{2}<b_{1} c_{2}, b_{1}<b_{2} c_{1}$, then the equilibrium solutions $E_{x}, E_{y}$ are locally asymptotically stable and the interior equilibrium $E$ is a saddle point. The separatrix $\ell_{S}$, which is a graph of a continuous, nondecreasing curve, is the basin of attraction of $E$ and the region below (resp. above) $\ell_{S}$ is the basin of attraction of $E_{x}$ (resp. $\left.E_{y}\right)$.

(b) If $b_{2}>b_{1} c_{2}, b_{1}>b_{2} c_{1}$, then the equilibrium solutions $E_{x}, E_{y}$ are saddle points and the interior equilibrium $E$ is locally asymptotically stable. Every solution in the first quadrant which starts off the coordinate axes converges to E. Every solution which starts on the positive part of the x-axis (resp. $y$-axis) is attracted by $E_{x}$ (resp. $\left.E_{y}\right)$.

(c) If $b_{2}<b_{1} c_{2}, b_{1}>b_{2} c_{1}$ (resp. $b_{2}>b_{1} c_{2}, b_{1}<b_{2} c_{1}$ ), then the equilibrium solution $E_{x}\left(r e s p . E_{y}\right)$ is locally asymptotically stable and $E_{y}\left(r e s p . E_{x}\right)$ is a saddle point. The basin of attraction of $E_{x}$ (resp. $\left.E_{y}\right)$ is the first quadrant of 
initial conditions without the positive part of the $y$-axis (resp. $x$-axis), which is attracted by $E_{y}$ (resp. $\left.E_{x}\right)$.

(d) If $b_{1}=b_{2} c_{1}$ and $b_{2}=b_{1} c_{2}$, then there is an infinite family of equilibrium solutions $E_{t}, t \in\left[0, b_{2}\right]$ for which there exists the global stable manifold $\mathcal{W}^{s}\left(E_{t}\right)$, which is the graph of a continuous, nondecreasing function asymptotic to $(0,0)$ and is exactly the basin of attraction of $E_{t}$. The limiting equilibrium varies continuously with the initial condition.

(e) If $E_{x}\left(\right.$ resp. $\left.E_{y}\right)$ is non-hyperbolic and $E_{y}$ (resp. $\left.E_{x}\right)$ is locally asymptotically stable, then $E_{y}$ (resp. $\left.E_{x}\right)$ attracts the first quadrant of initial conditions except the positive part of $x$-axis (resp. $y$-axis) which is attracted by $E_{x}$ (resp. $\left.E_{y}\right)$. If $E_{x}$ (resp. $\left.E_{y}\right)$ is non-hyperbolic and $E_{y}$ (resp. $\left.E_{x}\right)$ is a saddle point, then $E_{x}$ (resp. $E_{y}$ ) attracts the first quadrant of initial conditions except the positive part of the $y$-axis (resp. $x$-axis), which is attracted by $E_{y}$ (resp. $\left.E_{x}\right)$.

See Figure 2 for graphical interpretation.

Proof.

(a) First we show that $T$ does not have any period-two solutions. Our condition implies $c_{1} c_{2}>1$. By direct calculation one can show that a period-two solution satisfies the equation

$$
b_{1} b_{2}\left(c_{1} c_{2}-1\right) x^{2}+\left(b_{1} b_{2}\left(c_{1} c_{2}-1\right)+b_{1}^{2} c_{2}-b_{2}^{2} c_{1}\right) x+b_{1} b_{2}\left(b_{1}+b_{2} c_{1}\right)=0,
$$

which means that both terms of the solution are negative and so there is no period-two solution in the first quadrant.

Taking into account that the Jacobian matrix evaluated at $E$ has all nonzero entries, Theorem 5 of [16] implies the existence and uniqueness of both global 
stable and unstable manifolds, $\mathcal{W}^{s}(E)$ and $\mathcal{W}^{u}(E)$, and thus $\mathcal{W}^{s}(E)=\ell_{S}$. Furthermore, Theorem 5 of [16] implies that every $\left(x_{0}, y_{0}\right)$ below $\ell_{S}$ will satisfy $T^{n}\left(\left(x_{0}, y_{0}\right)\right) \in \llbracket E, E_{x} \rrbracket$ for some $n \geq N$. In view of Corollary 2, $T^{n}\left(\left(x_{0}, y_{0}\right)\right) \rightarrow E_{x}$. In a similar way, we can treat the case when $\left(x_{0}, y_{0}\right)$ is above $\ell_{S}$.

(b) In view of Lemma 2 part (a), the eigenvectors which correspond to $E_{x}$ and $E_{y}$ point to the interior of the fourth and the second quadrant, which means that the local unstable manifolds $\mathcal{W}_{l o c}^{u}\left(E_{x}\right)$ and $\mathcal{W}_{l o c}^{u}\left(E_{y}\right)$ exist and point strictly toward $E$. Thus, there exist points $u, v$ in the interior of $\llbracket E_{y}, E_{x} \rrbracket$, arbitrarily close to $E_{y}$ and $E_{x}$ such that $u \preceq_{s e} T(u) \preceq_{s e} E \preceq_{s e} T(v) \preceq_{s e} v$. Now, statement (iii) of Theorem 4 implies that $E$ is a global attractor in $\llbracket u, v \rrbracket$, which completes the proof.

(c) Assume that $b_{2}<b_{1} c_{2}, b_{1}>b_{2} c_{1}$, which implies that $E_{x}$ is locally asymptotically stable and $E_{y}$ is a saddle point. In view of Lemma 2 part (b), the eigenvector which corresponds to $E_{y}$ points to the interior of the fourth quadrant, which means that the local unstable manifold $\mathcal{W}_{l o c}^{u}\left(E_{y}\right)$ exists and points strictly toward $E_{x}$. Thus, there exists a point $u$ in the interior of $\llbracket E_{y}, E_{x} \rrbracket$, arbitrarily close to $E_{y}$ such that $u \preceq_{s e} T(u)$. Consequently, the map $T$ has a lower solution in every neighborhood of $E_{y}$, which in view of Theorem 6 in [4] implies that the interior of $\llbracket E_{y}, E_{x} \rrbracket$ is a subset of the basin of attraction of $E_{x}$. The result follows.

The proof when $b_{2}>b_{1} c_{2}, b_{1}<b_{2} c_{1}$ is similar and will be omitted. 
(d) By Theorem 1 of [4], for each $E_{t}$ there exists the set $\mathcal{W}^{s}\left(E_{t}\right)$ passing through $E_{t}$ and asymptotic to $(0,0)$, which is the graph of a continuous, nondecreasing function and is exactly the basin of attraction of $E_{t}$. The continuity of the limiting equilibrium solution as a function of initial conditions follows as in $[5]$.

(e) The proof is similar to the proof of part (c) and will be omitted.

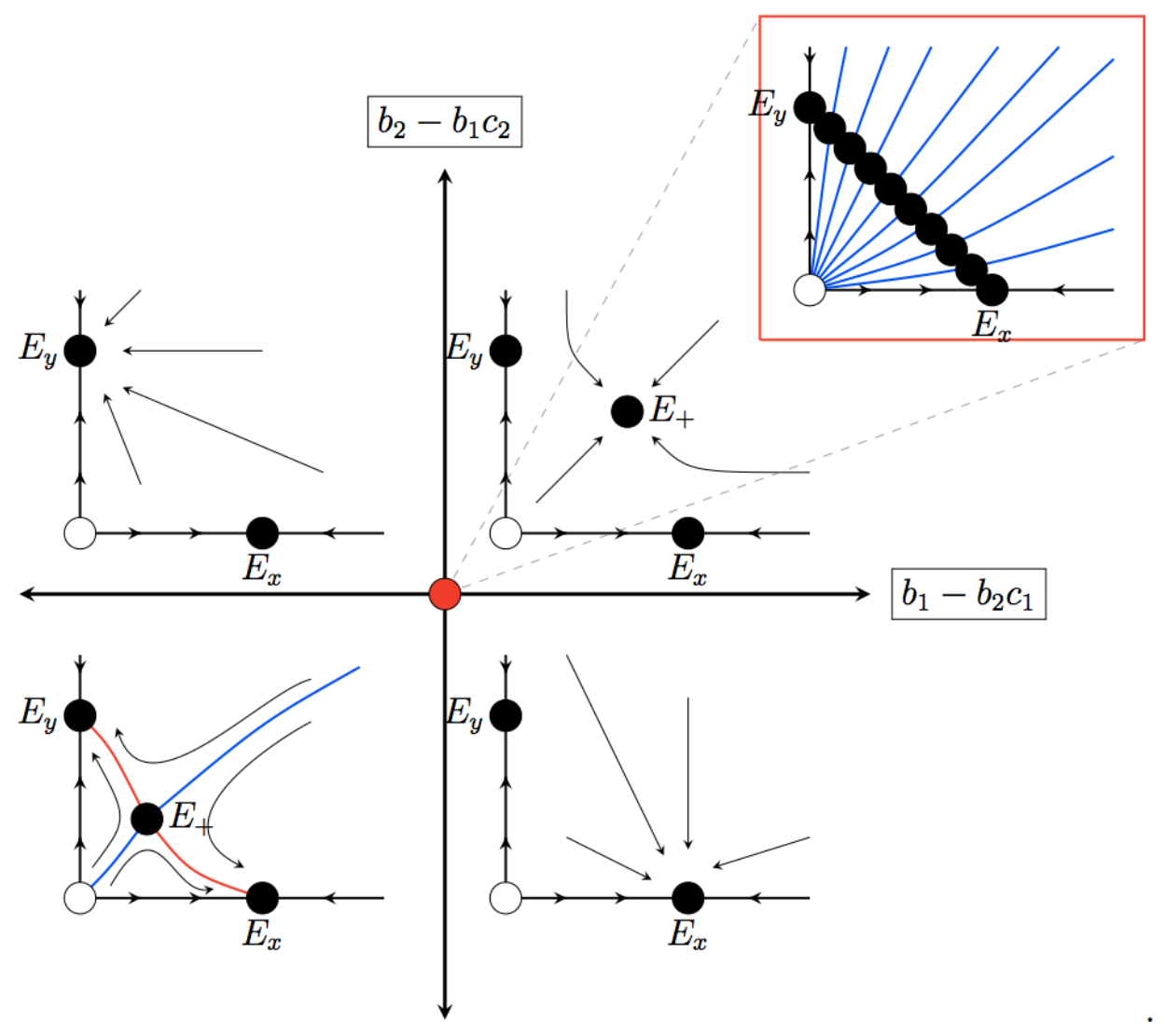

Figure 2: Global dynamics of system (4). 


\subsection{Global Dynamics of System (5)}

In this section we present the global behavior of system (5). Denote by

$$
\tilde{T}(x, y)=\left(\frac{b_{1} x}{\alpha_{1}+x+c_{1} y}, \frac{b_{2} y}{c_{2} x+y}\right),
$$

the map associated with system (5).

\section{Lemma 3 The following statements hold}

(a) Every solution of system (5) satisfies $x_{n}<b_{1}, y_{n} \leq b_{2}, \quad n \geq 1$.

(b) $\tilde{T}(x, y)$ satisfies $(O+)$ condition on $(0, \infty) \times(0, \infty)$ and so $T$ has no periodtwo points.

(c) For every $y_{0}>0, \tilde{T}\left(0, y_{0}\right)=\left(0, b_{2}\right)$.

(d) For every $x_{0}>0, \tilde{T}^{n}\left(x_{0}, 0\right)=\left(\frac{b_{1}-\alpha_{1}}{1-\left(\frac{\alpha_{1}}{b_{1}}\right)^{n}}, 0\right)$.

Proof. Part (a) and (c) follow by immediate checking and part (d) follows by solving the resulting Beverton-Holt one-dimensional equation. In light of Theorem 4 in [13], (b) can be proven by verifying that $\tilde{T}$ is injective and that $\operatorname{det}\left(J_{\tilde{T}}(x, y)\right)>0$ for all $(x, y) \in(0, \infty) \times(0, \infty)$. Notice

$$
\tilde{T}\left(\begin{array}{l}
x_{1} \\
y_{1}
\end{array}\right)=\tilde{T}\left(\begin{array}{l}
x_{2} \\
y_{2}
\end{array}\right) \Leftrightarrow\left(\begin{array}{c}
\frac{b_{1} x_{1}}{\alpha_{1}+x_{1}+c_{1} y_{1}} \\
\frac{b_{2} y_{1}}{c_{2} x_{1}+y_{1}}
\end{array}\right)=\left(\begin{array}{c}
\frac{b_{1} x_{2}}{\alpha_{1}+x_{2}+c_{1} y_{2}} \\
\frac{b_{2} 2}{c_{2} x_{2}+y_{2}}
\end{array}\right) .
$$

This is equivalent to

$$
x_{1} y_{2}=x_{2} y_{1}, \quad \alpha_{1}\left(x_{1}-x_{2}\right)=c_{1}\left(x_{2} y_{1}-x_{1} y_{2}\right) .
$$

It follows that $x_{1}=x_{2}, y_{1}=y_{2}$ and thus $\tilde{T}$ is injective. The Jacobian matrix associated with the map $\tilde{T}$ has the form

$$
J_{\tilde{T}}(x, y)=\left(\begin{array}{cc}
\frac{b_{1}\left(\alpha_{1}+c_{1} y\right)}{\left(\alpha_{1}+x+c_{1} y\right)^{2}} & -\frac{b_{1} c_{1} x}{\left(\alpha_{1}+x+c_{1} y\right)^{2}} \\
-\frac{b_{2} c_{2} y}{\left(c_{2} x+y\right)^{2}} & \frac{b_{2} c_{2} x}{\left(c_{2} x+y\right)^{2}}
\end{array}\right) .
$$


Therefore,

$$
\operatorname{det}\left(J_{\tilde{T}}(x, y)\right)=\frac{\alpha_{1} b_{1} b_{2} c_{2} x}{\left(c_{2} x+y\right)^{2}\left(\alpha_{1}+x+c_{1} y\right)^{2}}>0
$$

for all $(x, y) \in(0, \infty) \times(0, \infty)$.

Note that a direct consequence of the above form of $J_{\tilde{T}}(x, y)$ is that $\tilde{T}$ is strongly competitive. This will play a pivotal role in the global behavior of the system.

System (5) always has an equilibrium solution on the $y$-axis, $E_{y}\left(0, b_{2}\right)$. Provided that $\alpha_{1}<b_{1}$, there exists an equilibrium solution on the $x$-axis, $E_{x}\left(b_{1}-\alpha_{1}, 0\right)$. Depending on the values of the parameters $\alpha_{1}, b_{1}, b_{2}, c_{1}$ and $c_{2}$, there is also the possibility of either exactly one interior equilibrium solution $E$ or an infinite number of interior equilibrium solutions $E_{t}$.

The interior equilibrium solution is an intersection of two equilibrium curves $\mathcal{C}_{1}: \alpha_{1}+x+c_{1} y=b_{1}$ and $\mathcal{C}_{2}: c_{2} x+y=b_{2}$. This solution will exist if either $\frac{b_{2}}{c_{2}}<b_{1}-\alpha_{1}$ and $b_{2}>\frac{b_{1}-\alpha_{1}}{c_{1}}$ (i.e. the $x$-intercept of $\mathcal{C}_{2}$ is smaller than the $x$-intercept of $\mathcal{C}_{1}$ and the $y$-intercept of $\mathcal{C}_{2}$ is bigger than the $y$-intercept of $\mathcal{C}_{1}$ ) or $b_{1}-\alpha_{1}<\frac{b_{2}}{c_{2}}$ and $\frac{b_{1}-\alpha_{1}}{c_{1}}>b_{2}$ (i.e. the $x$-intercept of $\mathcal{C}_{1}$ is smaller than the $x$-intercept of $\mathcal{C}_{2}$ and the $y$-intercept of $\mathcal{C}_{1}$ is bigger than the $y$-intercept of $\mathcal{C}_{2}$ ). As in $(7)$, these two geometrical conditions can be unified as

$$
\Delta_{1} \Delta_{2}>0
$$

where $\Delta_{1}=\left(b_{2}-c_{2}\left(b_{1}-\alpha_{1}\right)\right)$ and $\Delta_{2}=\left(b_{1}-\alpha_{1}-b_{2} c_{1}\right)$. Condition (13) implies that $c_{1} c_{2} \neq 1$ and the interior equilibrium $E(\bar{x}, \bar{y})$ is given as:

$$
\bar{x}=\frac{b_{1}-\alpha_{1}-b_{2} c_{1}}{1-c_{1} c_{2}}, \quad \bar{y}=\frac{b_{2}-c_{2}\left(b_{1}-\alpha_{1}\right)}{1-c_{1} c_{2}} .
$$

Note that if $\Delta_{1} \Delta_{2}<0, \Delta_{1}=0$ and $\Delta_{2} \neq 0$, or $\Delta_{2}=0$ and $\Delta_{1} \neq 0$, then there does not exist an interior equilibrium solution. Since $\alpha_{1} \geq b_{1}$ implies that $\Delta_{1} \Delta_{2} \leq 0$, we must have $\alpha_{1}<b_{1}$ in order for an interior equilibrium point to exist. 
Furthermore, if $\Delta_{1}=\Delta_{2}=0$, then the two equilibrium curves $\mathcal{C}_{1}$ and $\mathcal{C}_{2}$ coincide and every point of the segment $c_{2} x+y=b_{2}, x, y \geq 0$ is an equilibrium solution $E_{t}\left(b_{1}-\alpha_{1}-c_{1} t, t\right), t \in\left[0, b_{2}\right]$. The equilibrium points for system (5) are summarized in Table 3.

\begin{tabular}{|c|c|c|}
\hline \multicolumn{2}{|c|}{ Condition } & Equilibrium Points \\
\hline \multicolumn{2}{|c|}{$\alpha_{1} \geq b_{1}$} & $E_{y}$ \\
\hline \multirow[t]{3}{*}{$\alpha_{1}<b_{1}$} & $\begin{array}{c}\Delta_{1} \Delta_{2}<0 \\
\Delta_{1}=0, \Delta_{2} \neq 0 \\
\Delta_{1} \neq 0, \Delta_{2}=0\end{array}$ & $E_{x}, E_{y}$ \\
\hline & $\Delta_{1} \Delta_{2}>0$ & $E_{x}, E_{y}, E$ \\
\hline & $\Delta_{1}=\Delta_{2}=0$ & $E_{x}, E_{y}, E_{t}$ \\
\hline
\end{tabular}

Table 3: The equilibrium points for system (5).

The local stability character of $E_{x}, E_{y}, E$ and $E_{t}$ are presented in Lemma 4. The proof requires Proposition 1.

Proposition 1 The eigenvalues $\lambda$ and $\mu$ of $J_{\tilde{T}}(E)$ are positive.

Proof. In view of (11) we have

$$
J_{\tilde{T}}(E)=\left(\begin{array}{cc}
\frac{b_{2} c_{1}-b_{1} c_{1} c_{2}+\alpha_{1}}{b_{1}\left(1-c_{1} c_{2}\right)} & -\frac{c_{1}\left(b_{1}-b_{2} c_{1}-\alpha_{1}\right)}{b_{1}\left(1-c_{1} c_{2}\right)} \\
-\frac{c_{2}\left(b_{2}-c_{2}\left(b_{1}-\alpha_{1}\right)\right)}{b_{2}\left(1-c_{1} c_{2}\right)} & \frac{c_{2}\left(b_{1}-b_{2} c_{1}-\alpha_{1}\right)}{b_{2}\left(1-c_{1} c_{2}\right)}
\end{array}\right),
$$

which implies

$$
\operatorname{det}\left(J_{\tilde{T}}(E)\right)=\frac{c_{2} \alpha_{1}\left(b_{1}-\alpha_{1}-b_{2} c_{1}\right)}{b_{1} b_{2}\left(1-c_{1} c_{2}\right)}
$$

Note that the equilibrium point $E$ exists under the hypothesis $\Delta_{1} \Delta_{2}>0$, which means that either $\frac{b_{2}}{c_{2}}<b_{1}-\alpha_{1}$ and $b_{2}>\frac{b_{1}-\alpha_{1}}{c_{1}}$ or $b_{1}-\alpha_{1}<\frac{b_{2}}{c_{2}}$ and $\frac{b_{1}-\alpha_{1}}{c_{1}}>b_{2}$. In either case, we have $\operatorname{det}\left(J_{\tilde{T}}(E)\right)>0$, and consequently, $\lambda \cdot \mu>0$. Since system (5) is strongly competitive, by the Perron-Frobenius Theorem [7, 18], the largest eigenvalue of $J_{\tilde{T}}(E)$ is real and positive. The result follows. 
Lemma 4 Consider system (5).

(a) The equilibrium solution $E_{x}$ exists if $\alpha_{1}<b_{1}$. It is locally asymptotically stable if $b_{2}<c_{2}\left(b_{1}-\alpha_{1}\right)$, non-hyperbolic of stable type if $b_{2}=c_{2}\left(b_{1}-\alpha_{1}\right)$, and a saddle point if $b_{2}>c_{2}\left(b_{1}-\alpha_{1}\right)$. In each case, the eigenvectors associated with the eigenvalues $\lambda_{1}=\frac{\alpha_{1}}{b_{1}}$ and $\lambda_{2}=\frac{b_{2}}{c_{2}\left(b_{1}-\alpha_{1}\right)}$ are $\mathrm{e}_{1}=(1,0)$ and $\mathrm{e}_{2}=$ $\left(\frac{c_{1} c_{2}\left(b_{1}-\alpha_{1}\right)^{2}}{c_{2} \alpha_{1}\left(b_{1}-\alpha_{1}\right)-b_{1} b_{2}}, 1\right)$, respectively.

(b) The equilibrium solution $E_{y}$ always exists and it is locally asymptotically stable if $b_{1}<\alpha_{1}+b_{2} c_{1}$, non-hyperbolic of stable type if $b_{1}=\alpha_{1}+b_{2} c_{1}$, and a saddle point if $b_{1}>\alpha_{1}+b_{2} c_{1}$. In each case, the eigenvectors associated with the eigenvalues $\lambda_{1}=0$ and $\lambda_{2}=\frac{b_{1}}{b_{2} c_{1}+\alpha_{1}}$ are $\mathrm{e}_{1}=(0,1)$ and $\mathrm{e}_{2}=$ $\left(\frac{b_{1}}{c_{2}\left(\alpha_{1}+b_{2} c_{1}\right)},-1\right)$, respectively.

(c) The interior equilibrium solution $E$ exists if $\Delta_{1} \Delta_{2}>0$ and it is locally asymptotically stable if $b_{1}>\alpha_{1}+b_{2} c_{1}$ and $b_{2}>c_{2}\left(b_{1}-\alpha_{1}\right)$ and a saddle point if $b_{1}<\alpha_{1}+b_{2} c_{1}$ and $b_{2}<c_{2}\left(b_{1}-\alpha_{1}\right)$.

(d) The interior equilibrium solutions $E_{t}$ exist if $\alpha_{1}<b_{1}, b_{1}=\alpha_{1}+b_{2} c_{1}$ and $b_{2}=$ $c_{2}\left(b_{1}-\alpha_{1}\right)$. They are non-hyperbolic of the stable type and the eigenvector associated with $\lambda_{1}$ where $\left|\lambda_{1}\right|<1$ is $\mathrm{e}_{1}=\left(\frac{\left(b_{1}-\alpha_{1}\right)\left(b_{2}-t\right)}{b_{1} c_{2} t}, 1\right)$.

Proof.

(a) In view of (11), we have

$$
J_{\tilde{T}}\left(E_{x}\right)=\left(\begin{array}{cc}
\frac{\alpha_{1}}{b_{1}} & -\frac{c_{1}\left(b_{1}-\alpha_{1}\right)}{b_{1}} \\
0 & \frac{b_{2}}{c_{2}\left(b_{1}-\alpha_{1}\right)}
\end{array}\right),
$$

which implies that the eigenvalues of the Jacobian matrix are $\lambda_{1}=\frac{\alpha_{1}}{b_{1}}, \lambda_{2}=$ $\frac{b_{2}}{c_{2}\left(b_{1}-\alpha_{1}\right)}$. The corresponding eigenvectors are as stated. 
(b) In view of (11), we have

$$
J_{\tilde{T}}\left(E_{y}\right)=\left(\begin{array}{cc}
\frac{b_{1}}{\alpha_{1}+b_{2} c_{1}} & 0 \\
-c_{2} & 0
\end{array}\right),
$$

which implies that the eigenvalues of the Jacobian matrix are $\lambda_{1}=0, \lambda_{2}=$ $\frac{b_{1}}{\alpha_{1}+b_{2} c_{1}}$. The corresponding eigenvectors are as stated.

(c) Denote the eigenvalues of $J_{\tilde{T}}(E)$ by $\lambda_{1}$ and $\lambda_{2}$, which represent the roots of the characteristic polynomial, $p(t)=t^{2}-\operatorname{tr} J_{\tilde{T}}(E) t+\operatorname{det} J_{\tilde{T}}(E)$. By Proposition $1, \lambda_{1}$ and $\lambda_{2}$ are real and positive. Notice

$$
\begin{gathered}
p(1)=\frac{\left(b_{1}-\alpha_{1}-b_{2} c_{1}\right)\left(b_{2}-c_{2}\left(b_{1}-\alpha_{1}\right)\right)}{b_{1} b_{2}\left(1-c_{1} c_{2}\right)}, \\
p^{\prime}(1)=\frac{b_{2}\left(b_{1}-\alpha_{1}-b_{2} c_{1}\right)+b_{1}\left(b_{2}-c_{2}\left(b_{1}-\alpha_{1}\right)\right)}{b_{1} b_{2}\left(1-c_{1} c_{2}\right)} .
\end{gathered}
$$

If $b_{1}<\alpha_{1}+b_{2} c_{1}$ and $b_{2}<c_{2}\left(b_{1}-\alpha_{1}\right)$, then $1<c_{1} c_{2}$ and by $(15), p(1)<0$. Combining this with the fact that $p(0)=\operatorname{det} J_{\tilde{T}}(E)>0$, it follows that $E$ is a saddle point. If $b_{1}>\alpha_{1}+b_{2} c_{1}$ and $b_{2}>c_{2}\left(b_{1}-\alpha_{1}\right)$, then $1>c_{1} c_{2}$ and from (15) and (16) we have $p(1)>0$ and $p^{\prime}(1)>0$. Combining this with $p(0)>0$, we conclude that $E$ is locally asymptotically stable.

(d) For $t \in\left[0, b_{2}\right)$, the eigenvalues of $J_{\tilde{T}}\left(E_{t}\right)$ are $\lambda_{1}=\frac{\alpha_{1}\left(b_{2}-t\right)}{b_{1} b_{2}}$ and $\lambda_{2}=1$. Since $\alpha_{1}<b_{1}$, we clearly have that $E_{t}$ are non-hyperbolic equilibrium points of the stable type. It follows by immediate checking that the eigenvector associated with $\lambda_{1}$ is $\mathrm{e}_{1}=\left(\frac{\left(b_{1}-\alpha_{1}\right)\left(b_{2}-t\right)}{b_{1} c_{2} t}, 1\right)$, which points towards the first quadrant for $t \in\left[0, b_{2}\right)$. 
The global behavior of system (5) is described by the following result. Note that the proofs presented for Theorem 6 differ from those of Theorem 5 in order to depict an alternative approach.

Theorem 6 Consider system (5).

(a) If $\alpha_{1} \geq b_{1}$, then $E_{y}$ is the unique equilibrium solution of system (5) and it is locally asymptotically stable. Every solution in the first quadrant which starts off of the $x$-axis converges to $E_{y}$ and every solution which starts on the positive $x$-axis converges to the singular point $(0,0)$.

(b) For $\alpha_{1}<b_{1}$, if $b_{1}>\alpha_{1}+b_{2} c_{1}, b_{2}<c_{2}\left(b_{1}-\alpha_{1}\right)$ (resp. $b_{1}<\alpha_{1}+b_{2} c_{1}$, $\left.b_{2}>c_{2}\left(b_{1}-\alpha_{1}\right)\right)$, then system (5) has equilibrium solutions $E_{x}$ and $E_{y}$ where $E_{x}\left(\right.$ resp. $\left.E_{y}\right)$ is locally asymptotically stable and $E_{y}$ (resp. $\left.E_{x}\right)$ is a saddle point. The basin of attraction of $E_{x}$ (resp. $\left.E_{y}\right)$ is the first quadrant of initial conditions without the positive part of the y-axis (resp. x-axis), which is attracted by $E_{y}$ (resp. $\left.E_{x}\right)$.

(c) If $\alpha_{1}<b_{1}, b_{1}>\alpha_{1}+b_{2} c_{1}$, and $b_{2}>c_{2}\left(b_{1}-\alpha_{1}\right)$, then system (5) has equilibrium solutions $E_{x}, E_{y}$ and $E$. The equilibrium solutions $E_{x}$ and $E_{y}$ are saddle points and $E$ is locally asymptotically stable. Every solution in the first quadrant which starts off of the coordinate axes converges to $E$ and every solution which starts on the positive $x$-axis (resp. $y$-axis) converges to $E_{x}\left(\right.$ resp. $\left.E_{y}\right)$.

(d) If $\alpha_{1}<b_{1}, b_{1}<\alpha_{1}+b_{2} c_{1}$, and $b_{2}<c_{2}\left(b_{1}-\alpha_{1}\right)$, then system (5) has equilibrium solutions $E_{x}, E_{y}$ and $E$. The equilibrium solutions $E_{x}$ and $E_{y}$ are locally asymptotically stable and the interior equilibrium $E$ is a saddle point. There exists the global stable manifold $\mathcal{W}^{s}(E)$ and the global unstable manifold $\mathcal{W}^{u}(E)$, where $\mathcal{W}^{s}(E)$ is the graph of a continuous, nondecreasing 
function and $\mathcal{W}^{u}(E)$ is the graph of a continuous, nonincreasing function which connects all three equilibrium solutions. The region in the first quadrant above (resp. below) the curve $\mathcal{W}^{s}(E)$ is the basin of attraction of $E_{y}$ (resp. $\left.E_{x}\right)$ and the curve $\mathcal{W}^{s}(E) \backslash\{(0,0)\}$ is the basin of attraction of $E$.

(e) If $\alpha_{1}<b_{1}, b_{1}=\alpha_{1}+b_{2} c_{1}$, and $b_{2}=c_{2}\left(b_{1}-\alpha_{1}\right)$, then there is an infinite family of equilibrium solutions $E_{t}$ for which there exists the global stable manifold $\mathcal{W}^{s}\left(E_{t}\right)$ for all $t \in\left[0, b_{2}\right]$, which is the graph of a continuous, nondecreasing function asymptotic to $(0,0)$ and is exactly the basin of attraction of $E_{t}$. The limiting equilibrium varies continuously with the initial condition.

(f) If $E_{x}\left(\right.$ resp. $\left.E_{y}\right)$ is non-hyperbolic and $E_{y}$ (resp. $\left.E_{x}\right)$ is locally asymptotically stable, then $E_{y}$ (resp. $E_{x}$ ) attracts the first quadrant of initial conditions except the positive part of the $x$-axis (resp. $y$-axis), which is attracted by $E_{x}$ (resp. $\left.E_{y}\right)$. If $E_{x}\left(\right.$ resp. $\left.E_{y}\right)$ is non-hyperbolic and $E_{y}$ (resp. $\left.E_{x}\right)$ is a saddle point, then $E_{x}$ (resp. $E_{y}$ ) attracts the first quadrant of initial conditions except the positive part of the $y$-axis (resp. $x$-axis), which is attracted by $E_{y}$ $\left(\operatorname{resp} . E_{x}\right)$.

See Figure 5 for graphical interpretation.

Proof.

(a) Let $\alpha_{1} \geq b_{1}$. Lemma 3(c) and 3(d) guarantee that for initial conditions on the positive $y$-axis, $\tilde{T}\left(x_{0}, y_{0}\right)=E_{y}$ and for initial conditions on the positive $x$-axis, $\lim _{n \rightarrow \infty} \tilde{T}^{n}\left(x_{0}, y_{0}\right)=(0,0)$. To treat the dynamics in the interior of $\mathbb{R}_{+}^{2}$, consider $\mathcal{R}_{a}:=\left\{(x, y): x, y \geq 0\right.$ and $\left.c_{2} x+y \leq b_{2}\right\}$. By Theorem 2 of [16], $\mathcal{R}_{a}$ is invariant. The region $\mathcal{R}_{a}$ also attracts the interior of $\mathbb{R}_{+}^{2}$. To verify this, suppose that $\left(x_{0}, y_{0}\right) \notin \mathcal{R}_{a}$ with $x_{0}, y_{0}>0$. In this case $c_{2} x_{0}+y_{0}>b_{2}$ 
and

$$
x_{1}=\frac{b_{1} x_{0}}{\alpha_{1}+x_{0}+c_{1} y_{0}}<x_{0} \quad \text { and } \quad y_{1}=\frac{b_{2} y_{0}}{c_{2} x_{0}+y_{0}}<y_{0} .
$$

It follows that there exists $N>0$ such that for all $n \geq N,\left(x_{n}, y_{n}\right) \in \mathcal{R}_{a}$ and thus $\mathcal{R}_{a}$ is attracting. To conclude the proof, suppose $\left(x_{0}, y_{0}\right) \in \mathcal{R}_{a}$ with $x_{0}, y_{0}>0$. In this case $x_{1}<x_{0}, y_{1} \geq y_{0}$ and as a consequence of the invariance of $\mathcal{R}_{a},\left\{x_{n}\right\}$ is a decreasing sequence while $\left\{y_{n}\right\}$ is a nondecreasing sequence. Therefore, $\lim _{n \rightarrow \infty} \tilde{T}^{n}\left(x_{0}, y_{0}\right)=E_{y}$. The above arguments prove that the basins of attraction for $E_{y}$ and the singular point $(0,0)$ are $\mathcal{B}\left(E_{y}\right)=$ $[0, \infty) \times(0, \infty)$ and $\mathcal{B}(0,0)=[0, \infty) \times\{0\}$

(b) Let $\alpha_{1}<b_{1}, b_{1}>\alpha_{1}+b_{2} c_{1}$, and $b_{2}<c_{2}\left(b_{1}-\alpha_{1}\right)$. Lemma 3(c) and 3(d) guarantee that for all initial conditions on the positive $y$-axis, $\tilde{T}\left(x_{0}, y_{0}\right)=E_{y}$ and for all initial conditions on the positive $x$-axis, $\lim _{n \rightarrow \infty} \tilde{T}^{n}\left(x_{0}, y_{0}\right)=E_{x}$. To treat the interior of $\mathbb{R}_{+}^{2}$, consider $\mathcal{R}_{b}:=\left\{(x, y): x, y \geq 0, c_{2} x+y \geq\right.$ $b_{2}$ and $\left.\alpha_{1}+x+c_{1} y \leq b_{1}\right\}$ shown in Figure 3 .

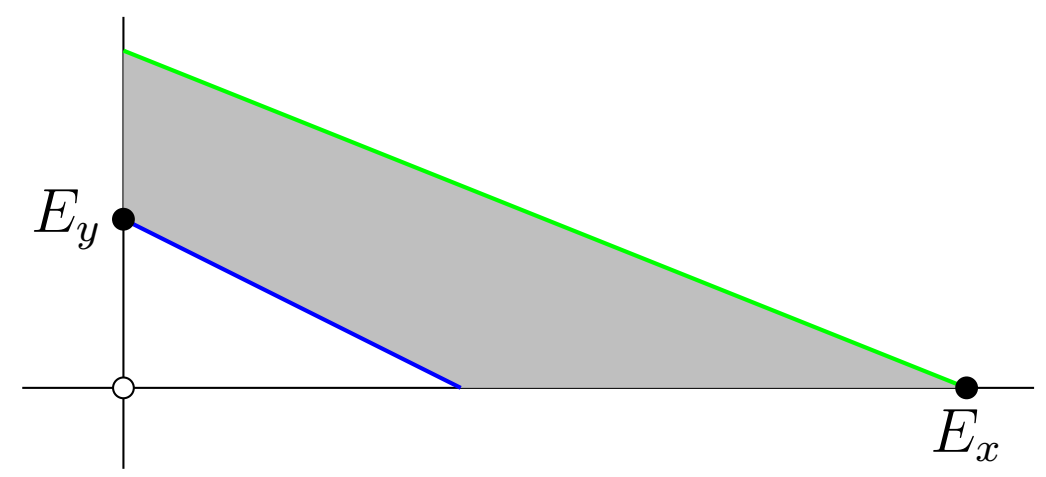

Figure $3: \mathcal{R}_{b}:=\left\{(x, y): x, y \geq 0, c_{2} x+y \geq b_{2}\right.$ and $\left.\alpha_{1}+x+c_{1} y \leq b_{1}\right\}$

Note that $\mathcal{R}_{b}$ is an invariant region by Theorem 2 of [16]. Consider $\left(x_{0}, y_{0}\right) \in$ $\mathcal{R}_{b}$ with $x_{0}, y_{0}>0$ and notice

$$
x_{1}=\frac{b_{1} x_{0}}{\alpha_{1}+x_{0}+c_{1} y_{0}} \geq x_{0} \quad \text { and } \quad y_{1}=\frac{b_{2} y_{0}}{c_{2} x_{0}+y_{0}} \leq y_{0}
$$


As a consequence of the invariance of $\mathcal{R}_{b},\left\{x_{n}\right\}$ is a nondecreasing sequence and $\left\{y_{n}\right\}$ is a nonincreasing sequence. Therefore, using basic properties of sequences and the fact that $\tilde{T}$ is strongly competitive, $\lim _{n \rightarrow \infty} \tilde{T}^{n}\left(x_{0}, y_{0}\right)=E_{x}$. Finally, suppose $\left(x_{0}, y_{0}\right) \notin \mathcal{R}_{b}$ with $x_{0}, y_{0}>0$. By Lemma $3(\mathrm{a}),\left(x_{n}, y_{n}\right) \in$ $\left[0, b_{1}\right) \times\left[0, b_{2}\right]$ for all $n \geq 1$. Choose $(u, v) \in \mathcal{R}_{b}$ with $u, v>0$ such that $(u, v) \preceq_{s e}\left(x_{1}, y_{1}\right) \preceq_{s e}\left(x_{1}, 0\right)$. Since $\tilde{T}$ is strongly competitive, notice

$$
\tilde{T}^{n}(u, v) \ll_{s e} \tilde{T}^{n}\left(x_{1}, y_{1}\right) \ll_{s e} \tilde{T}^{n}\left(x_{1}, 0\right) .
$$

Therefore, $\lim _{n \rightarrow \infty} \tilde{T}^{n}\left(x_{0}, y_{0}\right)=E_{x}$. We have arrived at the desired result that the basins of attraction for $E_{x}$ and $E_{y}$ are $\mathcal{B}\left(E_{x}\right)=(0, \infty) \times[0, \infty)$ and $\mathcal{B}\left(E_{y}\right)=\{0\} \times(0, \infty)$.

The proof for the case when $b_{1}<\alpha_{1}+b_{2} c_{1}, b_{2}>c_{2}\left(b_{1}-\alpha_{1}\right)$ is similar and will be omitted.

(c) Let $\alpha_{1}<b_{1}, b_{1}>\alpha_{1}+b_{2} c_{1}$, and $b_{2}>c_{2}\left(b_{1}-\alpha_{1}\right)$. As in part (b), Lemma 3(c) and $3(\mathrm{~d})$ guarantee that the positive part of the $y$-axis is a subset of $\mathcal{B}\left(E_{y}\right)$ and the positive part of the $x$-axis is a subset of $\mathcal{B}\left(E_{x}\right)$. To treat the interior of $\mathbb{R}_{+}^{2}$, consider the region $\mathcal{R}_{c}$ shown in Figure 4 .

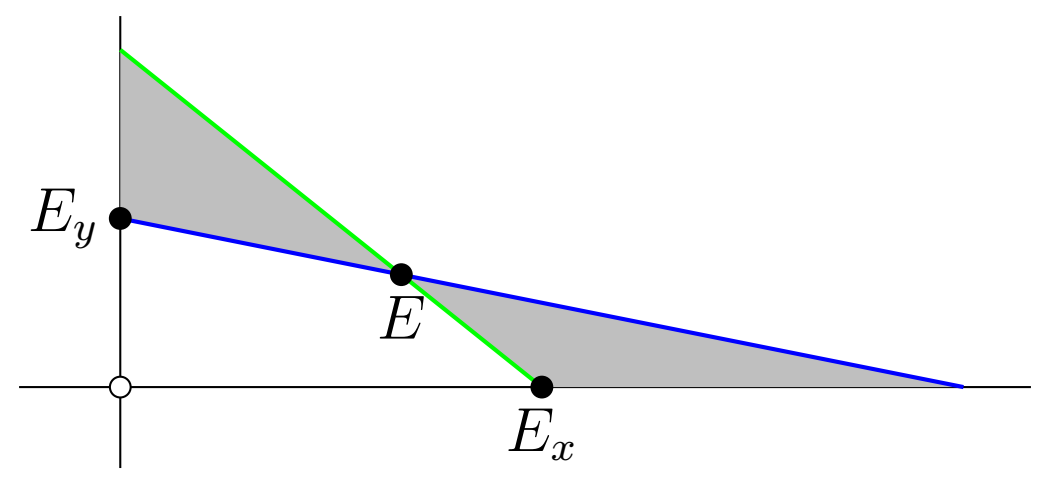

Figure 4: $\mathcal{R}_{c}:=\left\{(x, y): x, y \geq 0\right.$ and $\left.\left(x+c_{1} y+\alpha_{1}-b_{1}\right)\left(c_{2} x+y-b_{2}\right) \leq 0\right\}$

Note that $\mathcal{R}_{c}$ is invariant by Theorem 2 of [16]. Provided that $\left(x_{0}, y_{0}\right) \in \mathcal{R}_{c}$ with $x_{0}, y_{0}>0$, monotonicity properties (similar to part (a) and (b)) 
along with Lemma $3(\mathrm{~b})$ can be used to prove that $\lim _{n \rightarrow \infty} \tilde{T}^{n}\left(x_{0}, y_{0}\right)=E$. Suppose $\left(x_{0}, y_{0}\right) \notin \mathcal{R}_{c}$ with $x_{0}, y_{0}>0$. By Lemma 3(a) we know that $\left(x_{n}, y_{n}\right) \in\left[0, b_{1}\right) \times\left[0, b_{2}\right]$ for all $n \geq 1$. Moreover, since $\left(x_{0}, y_{0}\right) \preceq_{s e}\left(x_{0}, 0\right)$, then $\tilde{T}^{n}\left(x_{0}, y_{0}\right) \ll_{s e} \tilde{T}^{n}\left(x_{0}, 0\right)$ for all $n \geq 1$. Consequently, there must exist an $N \geq 1$ such that $\left(x_{N}, y_{N}\right) \in\left[0, \frac{b_{2}}{c_{2}}\right) \times\left[0, b_{2}\right]$. Now, choose $(u, v),(s, t) \in \mathcal{R}_{c}$ such that $(u, v) \preceq_{s e}\left(x_{N}, y_{N}\right) \preceq_{s e}(s, t)$. Since $\tilde{T}$ is strongly competitive, we have

$$
\tilde{T}^{n}(u, v) \ll_{s e} \tilde{T}^{n}\left(x_{N}, y_{N}\right) \ll_{s e} \tilde{T}^{n}(s, t) .
$$

Therefore, $\lim _{n \rightarrow \infty} \tilde{T}^{n}\left(x_{0}, y_{0}\right)=E$ for all $\left(x_{0}, y_{0}\right) \notin \mathcal{R}_{c}$. We have reached the desired result that the basins of attraction for $E, E_{x}$ and $E_{y}$ are $\mathcal{B}(E)=$ $(0, \infty) \times(0, \infty), \mathcal{B}\left(E_{x}\right)=(0, \infty) \times\{0\}$ and $\mathcal{B}\left(E_{y}\right)=\{0\} \times(0, \infty)$

(d) Let $\alpha_{1}<b_{1}, b_{1}<\alpha_{1}+b_{2} c_{1}$, and $b_{2}<c_{2}\left(b_{1}-\alpha_{1}\right)$. In light of Lemma 4(d), Theorem 1 of [4] guarantees that there exist the global stable and unstable manifolds for $E, \mathcal{W}^{s}(E)$ and $\mathcal{W}^{u}(E)$, respectively, with the above mentioned properties. An immediate checking shows that $E_{y} \preceq_{s e} E \preceq_{s e} E_{x}$ and that the interior of the ordered interval $\llbracket E_{y}, E \rrbracket$ is a subset of $\mathcal{B}\left(E_{y}\right)$, while the interior of the ordered interval $\llbracket E, E_{x} \rrbracket$ is a subset of $\mathcal{B}\left(E_{x}\right)$. Now, take any point $\left(x_{0}, y_{0}\right) \in \mathbb{R}_{+}^{2}$ such that $\left(x_{0}, y_{0}\right) \prec_{s e} \mathcal{W}^{s}(E)$ (i.e. above $\left.\mathcal{W}^{s}(E)\right)$. Then $\left(0, y_{0}\right) \preceq_{s e}\left(x_{0}, y_{0}\right) \prec_{s e}\left(x_{\mathcal{W}^{s}(E)}, y_{0}\right)$, where $\left(x_{\mathcal{W}^{s}(E)}, y_{0}\right) \in \mathcal{W}^{s}(E)$. By Lemma 3 (c) and the monotonicity of $\tilde{T}$, for $n \geq 1$,

$$
E_{y}=\tilde{T}^{n}\left(\left(0, y_{0}\right)\right) \ll_{s e} \tilde{T}^{n}\left(\left(x_{0}, y_{0}\right)\right) \ll_{s e} \tilde{T}^{n}\left(\left(x_{\mathcal{W}^{s}(E)}, y_{0}\right)\right)
$$

Since $\lim _{n \rightarrow \infty} \tilde{T}^{n}\left(\left(x_{\mathcal{W}^{s}(E)}, y_{0}\right)\right)=E,(19)$ implies that $\tilde{T}^{n}\left(\left(x_{0}, y_{0}\right)\right)$ enters the ordered interval $\llbracket E_{y}, E \rrbracket$ and so converges to $E_{y}$. In a similar way, one can show that the ordered interval $\llbracket E, E_{x} \rrbracket$ attracts all points below $\mathcal{W}^{s}(E)$, and so all such points converge to $E_{x}$. 
(e) By Theorem 1 of [4], for each $E_{t}$ there exists the set $\mathcal{W}^{s}\left(E_{t}\right)$ passing through $E_{t}$ and asymptotic to $(0,0)$, which is the graph of a continuous, nondecreasing function and is exactly the basin of attraction of $E_{t}$. The continuity of the limiting equilibrium solution as a function of initial conditions follows as in $[5]$.

(f) The proof is similar to the proof of part (b) and will be omitted here.

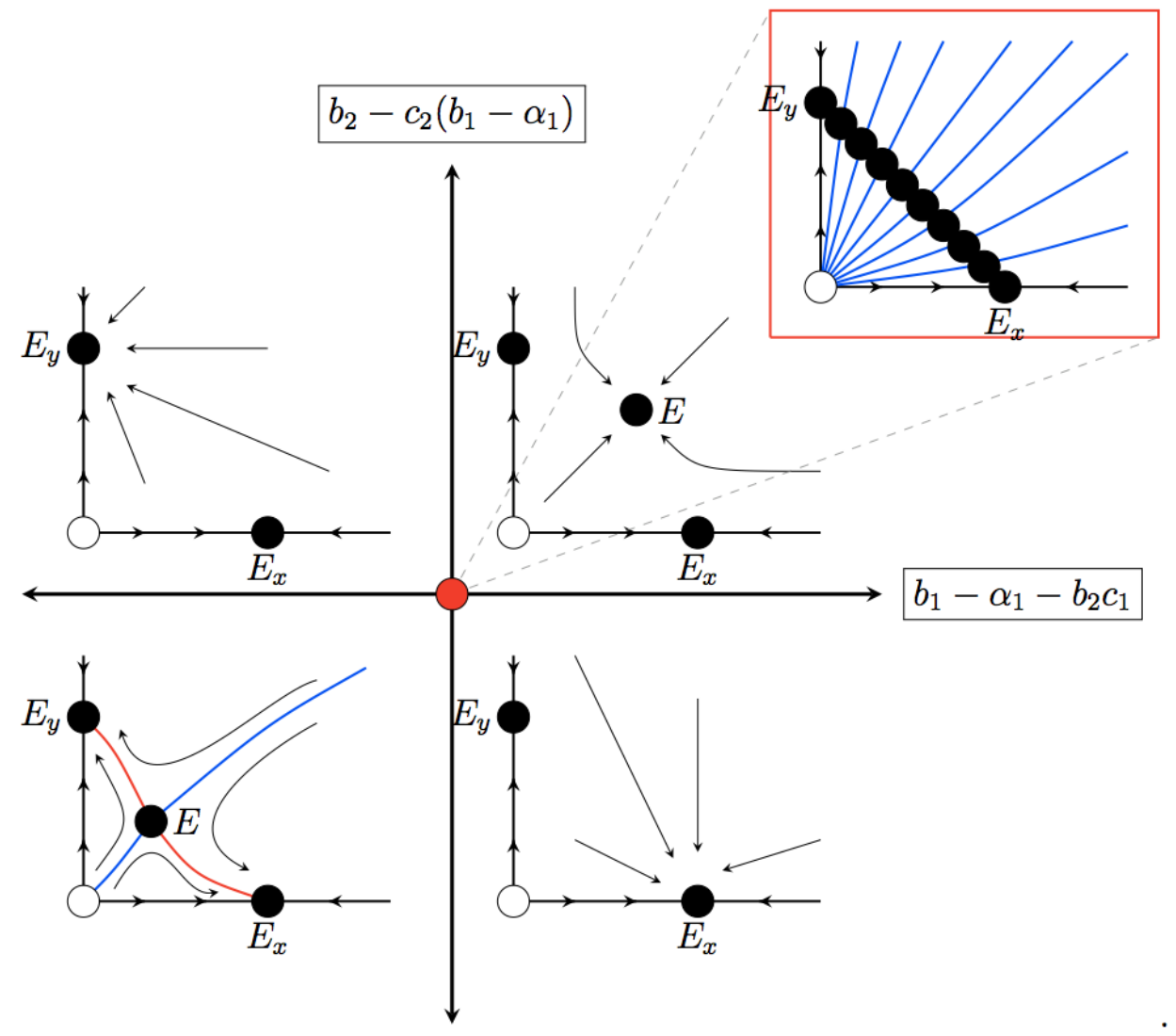

Figure 5: Global dynamics of system (5).

Based on Figures 2 and 5, the global dynamics of systems (4) and (5) are similar. However, the techniques of the proofs are different since the determinant of the map corresponding to system (4) is identically zero in the first quadrant, 
while the determinant of the map corresponding to (5) is positive and the map satisfies $(O+)$ condition. This condition greatly simplifies the proof for system (5). The qualitative difference between system (1) and systems (4) and (5) is in the case when $b_{1} \leq 1, b_{2} \leq 1$. In this case, $E_{0}(0,0)$ is a globally asymptotically stable equilibrium for system (1), the basin of attraction of the singular point $E_{0}(0,0)$ is an empty set for system $(4)$, and the basin of attraction of the singular point $E_{0}(0,0)$ is the nonnegative part of the $x$-axis for system (5). Furthermore, while system (4) always possesses two equilibrium solutions, systems (1) and (5) possess only one equilibrium solution for all parameter regions.

\subsection{Global Dynamics Scenarios for Competitive Systems}

In this section we give some general results about the global dynamics of a general competitive system (6). The proofs are analogous to the ones given in Theorems 5 and 6 and will be ommited.

Theorem 7 Consider the competitive map $T$ associated with system (6).

(a) Assume that $T$ has a saddle fixed point $E_{x}$, locally asymptotically stable point $E_{y}$, and either another repelling fixed point or a singular point $E_{0}$, which is a South-West corner of the region $\mathcal{R}$ and satisfies $E_{y} \preceq_{s e} E_{0} \preceq_{s e} E_{x}$. If $T$ has no period-two solutions, then every solution which starts off of the $x$-axis converges to $E_{y}$ and every solution which starts on the positive part of the $x$-axis converges to $E_{x}$.

(b) Assume that $T$ has a saddle fixed point $E_{y}$, locally asymptotically stable point $E_{x}$, and either another repelling fixed point or a singular point $E_{0}$, which is a South-West corner of the region $\mathcal{R}$ and satisfies $E_{y} \preceq_{s e} E_{0} \preceq_{s e} E_{x}$. If $T$ has no period-two solutions, then every solution which starts off of the y-axis converges to $E_{x}$ and every solution which starts on the positive part of the 
$y$-axis converges to $E_{y}$.

(c) Assume that $T$ has three fixed points $E_{x}, E_{y}, E$ such that $E_{y} \preceq_{\text {se }} E \preceq_{\text {se }}$ $E_{x}$, where $E_{x}, E_{y}$ are saddle points, and $E$ is locally asymptotically stable. Assume that $T$ has either another repelling fixed point or a singular point $E_{0}$, which is a South-West corner of the region $\mathcal{R}$ and satisfies $E_{y} \preceq_{s e} E_{0} \preceq_{s e} E_{x}$. If $T$ has no period-two solutions, then every solution which starts in the interior of $\mathcal{R}$ converges to E. Every solution which starts on the positive part of the $x$-axis (resp. y-axis) converges to $E_{x}$ (resp. $\left.E_{y}\right)$.

(d) Assume that $T$ has three fixed points $E_{x}, E_{y}, E$ such that $E_{y} \preceq_{s e} E \preceq_{s e} E_{x}$, where $E_{x}, E_{y}$ are locally asymptotically stable, and $E$ is a saddle point. Assume that $T$ has either another repelling fixed point or a singular point $E_{0}$, which is a South-West corner of the region $\mathcal{R}$ and satisfies $E_{y} \preceq_{\text {se }} E_{0} \preceq_{\text {se }} E_{x}$. If $T$ has no period-two solutions, then there exist the global stable and unstable manifolds $\mathcal{W}^{s}(E)$ and $\mathcal{W}^{u}(E)$ passing through $E$. The stable manifold $\mathcal{W}^{s}(E)$ has one endpoint at $E_{0}$ and is the graph of a continuous, nondecreasing function. The unstable manifold $\mathcal{W}^{u}(E)$ has endpoints at $E_{x}$ and $E_{y}$ and is the graph of a continuous, nonincreasing function. Every solution which starts below (resp. above) the stable manifold $\mathcal{W}^{s}(E)$ converges to $E_{x}$ (resp. $\left.E_{y}\right)$. Every point which starts on $\mathcal{W}^{s}(E) \backslash\left\{E_{0}\right\}$ converges to $E$.

(e) Assume that $T$ has an infinite number of fixed points which belong to the arc of a continuous decreasing curve $\mathcal{C}$ with end points $E_{x}$ and $E_{y}$ on the $x$ and $y$-axis respectively. Assume that $T$ has either another repelling fixed point or a singular point $E_{0}$, which is a South-West corner of the region $\mathcal{R}$. If $T$ has no period-two solutions, then every solution which starts in the first quadrant belongs to exactly one nondecreasing, continuous curve $\mathcal{W}^{s}\left(E_{\mathcal{C}}\right)$ that crosses 
$\mathcal{C}$ at the point $E_{\mathcal{C}}$ and either has an endpoint at $E_{0}$ or is asymptotic to $E_{0}$.

The dynamic behavior described in (a), (b), and (d) of Theorem 7 are called competitive exclusion scenarios and the dynamic behavior described in (c) of Theorem 7 is called a competitive coexistence scenario.

\section{Acknowledgements.}

M. R. S. Kulenović is supported in part by Maitland P. Simmons Foundation.

\section{Competing interests}

The authors declare that they have no competing interests.

\section{Authors contributions}

All the authors contributed equally to this work. They all read and approved the final version of the manuscript.

\section{List of References}

[1] J. Cushing, S. Levarge, N. Chitnis, , and S. Henson, "Some discrete competition models and the competitive exclusion principle," Journal of Difference Equations and Applications, vol. 10, pp. 1139-1151, 2004.

[2] M. R. S. Kulenović and O. Merino, "Global bifurcation for competitive systems in the plane," Discrete Contin. Dyn. Syst. Ser. B, vol. 12, pp. 133-149, 2009.

[3] E. Camouzis, M. R. S. Kulenović, G. Ladas, and O. Merino, "Rational systems in the plane - open problems and conjectures," Journal of Difference Equations and Applications, vol. 15, pp. 303-323, 2009.

[4] M. R. S. Kulenović and O. Merino, "Invariant manifolds for competitive discrete systems in the plane," Int. J. of Bifurcations and Chaos, vol. 20, pp. 2471-2486, 2010.

[5] D. Burgić, S. Kalabušić, and M. R. S. Kulenović, "Non-hyperbolic dynamics for competitive systems in the plane and global period-doubling bifurcations," Adv. Dyn. Syst. Appl., vol. 3, pp. 229-249, 2008. 
[6] D. Clark, M. R. S. Kulenović, and J. F. Selgrade, "Global asymptotic behavior of a two dimensional difference equation modelling competition," Nonlinear Anal., vol. 52, pp. 1765-1776, 2003.

[7] P. Hess, Periodic-parabolic boundary value problems and positivity. New York: Longman Scientific \& Technical, Harlow, 1991.

[8] S. Kalabušić, M. R. S. Kulenović, and E. Pilav, "Dynamics of a twodimensional system of rational difference equations of leslie-gower type," Advances in Difference Equations, vol. 29, pp. 1-29, 2011.

[9] M. R. S. Kulenović and O. Merino, Discrete Dynamical Systems and Difference Equations with Mathematica. Boca Raton: Chapman\& Hall/CRC Press, 2002.

[10] M. R. S. Kulenović and M. Nurkanović, "Asymptotic behavior of a linear fractional system of difference equations," J. Ineq. Appl., vol. 1, pp. 127-143, 2005.

[11] W. J. Leonard and R. May, "Nonlinear aspects of competition between species," SIAM J. Appl. Math., vol. 29, pp. 243-275, 1975.

[12] S. Smale, "On the differential equations of species in competition," Journal of Mathematical Biology, vol. 3, pp. 5-7, 1976.

[13] H. L. Smith, "Planar competitive and cooperative difference equations," Journal of Difference Equations and Applications, vol. 3, pp. 335-357, 1998.

[14] A. Bilgin, M. R. S. Kulenović, and E. Pilav, "Basins of attraction of period-two solutions of monotone systems of difference equations," Advances in Difference Equations, vol. 74, pp. 1-25, 2016.

[15] H. L. Smith, "Periodic competitive differential equations and the discrete dynamics of competitive maps," Journal of Differential Equations, vol. 64, pp. 165-194, 1986.

[16] M. R. S. Kulenović and O. Merino, "Competitive-exclusion versus competitive-coexistence for systems in the plane," Discrete Contin. Dyn. Syst. Ser. B, vol. 6, pp. 1141-1156, 2006.

[17] Y. S. Huang and P. M. Knopf, "Global convergence properties of first-order homogeneous systems of rational difference equations," Journal of Difference Equations and Applications, vol. 18, pp. 1683-1707, 2012.

[18] M. Hirsch and H. Smith, Handbook of Differential Equations: Ordinary Differential Equations; Monoton Dynamical Systems. Amsterdam: Elsevier B. V., 2005. 


\section{MANUSCRIPT 3 \\ The Global Dynamics and Boundedness of a Host-Parasite Model}

David T. McArdle and Orlando Merino

Publication Status:

Submitted March 23rd, 2017, Journal of Difference Equations and Applications

Keywords: boundedness, difference equation, global asymptotic stability, host parasitoid, host parasite, persistence

AMS Subject Classification: 39A10, 39A22 


\section{Abstract}

We consider the system of difference equations

$$
x_{n+1}=\frac{\alpha x_{n}}{1+\beta y_{n}}, \quad y_{n+1}=\frac{\gamma x_{n} y_{n}}{x_{n}+\delta y_{n}}, \quad n=0,1,2, \ldots,
$$

where $\alpha, \beta, \gamma, \delta, x_{0}, y_{0}$ are positive real numbers. This system was formulated by P. H. Leslie in 1948 and the present manuscript provides the most complete dynamical analysis to date. A boundedness and persistence result along with global attractivity results for various parameter regions are established. Numerical evidence of chaotic behavior is also presented for solutions of the system in select parameter regions.

\subsection{Introduction}

A host-parasitoid model is a type of prey-predator model where the development of the attacking species (parasitoid) depends on the quantity of the food species (host) made available to it and the population of the food species depends on how many of its peers survived the infestation $[1,2]$. A parasitoid is a term that refers to a parasite that lives in an environment and lays eggs in the larvae or pupae of the host population $[1,3]$. Once a host is parasitized, it dies off but the eggs of the parasitoid may continue to the next generation $[1,3]$. Often parasitoids are strategically used as biological pest control agents to kill off unwanted insect populations and applications can be found in [1], [4], [5], and [6]. The general framework for describing the dynamics of such a model is

$$
x_{n+1}=a x_{n} f\left(x_{n}, y_{n}\right), \quad y_{n+1}=c x_{n}\left(1-f\left(x_{n}, y_{n}\right)\right), \quad n=0,1,2, \ldots,
$$

where $x_{n}$ and $y_{n}$ represent the size of the host and parasitoid populations in the $n^{\text {th }}$ generation, respectively, and $f\left(x_{n}, y_{n}\right)$ represents the fraction of hosts that survive the parasitoid $[2,4]$. Many authors have studied host-parasitoid models with the 
general form given in (1). See [7], [4], [3], [8], [9], [10], and [11] for an analysis of such models.

Host-parasite models have a similar structure to that of host-parasitoid models with the biggest difference being that the parasite may not kill the host $[1,12]$. These models have attracted the attention of many authors in recent years and several interesting systems are studied in [13], [14], [15], [16] and [17]. One hostparasite model of particular interest, formulated in 1948 by P. H. Leslie, is given by

$N_{1}(t+1)=\frac{\lambda_{1} N_{1}(t)}{1+\left(\lambda_{1}-1\right) \frac{N_{2}(t)}{K_{2}}}, \quad N_{2}(t+1)=\frac{\lambda_{2} N_{2}(t)}{1+\left(\lambda_{2}-1\right) \frac{K_{1} N_{2}(t)}{K_{2} N_{1}(t)}}, \quad t=0,1,2, \ldots$,

where $\lambda_{1}, \lambda_{2}>1$ and $K_{1}, K_{2}$ are positive constants (see page 239 in [18]). The quantity $N_{1}$ represents the population of the host and $N_{2}$ represents the population of the parasite. An increase in the parasite population $N_{2}$ results in a decrease in the host population $N_{1}$ and an increase in the ratio $\frac{N_{2}}{N_{1}}$ results in a decrease in the parasite population as they lack resources to survive. See [18, 19] for more information on (2). System (2) can be rewritten as

$$
x_{n+1}=\frac{\alpha x_{n}}{1+\beta y_{n}}, \quad y_{n+1}=\frac{\gamma x_{n} y_{n}}{x_{n}+\delta y_{n}}, \quad n=0,1,2, \ldots
$$

where $\alpha, \beta, \gamma, \delta, x_{0}, y_{0}$ are positive real numbers.

System (3) has been studied by Q. Din and T. Donchev, who claim in Theorem 6 of [12] that when $\alpha, \gamma>1$ the unique positive equilibrium is a global attractor. However, the proof in [12] is incorrect as we now explain. The analysis of system (3) in [12] relies on Theorem 5 of [12], which is a result that appeared first as Theorems 2.2.9 and 2.2.11 in the PhD thesis of M. Nurkanović [20]. Also see [21]. A generalization of these results is Theorem 3 in [22]. The result of Nurkanović (or Theorem 3 of [22]) guarantees boundedness and persistence of solutions to (3) 
on sets $\left[m_{1}, M_{1}\right] \times\left[m_{2}, M_{2}\right]$ that are invariant under the map associated with the system. The purported proof of Theorem 6 in [12] failed to verify that nontrivial invariant sets $\left[m_{1}, M_{1}\right] \times\left[m_{2}, M_{2}\right]$ exist, and therefore global attractivity of the equilibrium was not established. In fact, no such sets exist: if $\left[m_{1}, M_{1}\right] \times\left[m_{2}, M_{2}\right]$ is an invariant subset of the positive quadrant of the plane, then by monotonicity and invariance,

$$
m_{1} \leq \frac{\alpha m_{1}}{1+\beta M_{2}} \quad \text { and } \quad \frac{\alpha M_{1}}{1+\beta m_{2}} \leq M_{1}
$$

From (4), one obtains $1+\beta M_{2} \leq \alpha$ and $\alpha \leq 1+\beta m_{2}$, hence $m_{2}=M_{2}$. A similar calculation gives $m_{1}=M_{1}$, and it follows that the invariant set consists of just one point. Consequently, Nurkanović's result cannot be used to prove that the positive equilibrium in (3) is a global attractor. The present manuscript provides a proof, among other things, of the global attractivity of the unique positive equilibrium as well as the boundedness and persistence of solutions to system (3) under certain parameter restrictions that include those considered by Din and Donchev. The results in the coming sections provide the most complete analysis to date of model (2) formulated by P. H. Leslie in 1948.

Before we state the main result of this paper, it is convenient to introduce the change of variables

$$
x^{\prime}=\frac{\beta}{\delta} x, \quad y^{\prime}=\beta y .
$$

This change of variables allows for the elimination of the parameters $\beta$ and $\delta$, and after renaming variables, system (3) is transformed to

$$
x_{n+1}=\frac{\alpha x_{n}}{1+y_{n}}, \quad y_{n+1}=\frac{\gamma x_{n} y_{n}}{x_{n}+y_{n}}, \quad n=0,1,2, \ldots
$$

An elementary calculation gives that a positive equilibrium for (5) exists if and only if $\alpha>1$ and $\gamma>1$. When this equilibrium exists, it is unique and given by

$$
\left(\bar{x}_{+}, \bar{y}_{+}\right):=\left(\frac{\alpha-1}{\gamma-1}, \alpha-1\right) .
$$


It is worth pointing out that whenever the positive equilibrium (6) exists, it is locally asymptotically stable. This can be seen from the characteristic polynomial $g(t)$ of the Jacobian matrix of the map associated with (5) evaluated at the equilibrium (6), which is given by

$$
g(t)=t^{2}+p t+q=t^{2}-\frac{\gamma+1}{\gamma} t+\frac{1+(\alpha-1) \gamma}{\alpha \gamma}
$$

Rather than calculating the roots of $g(t)$ explicitly, we proceed to verify the SchurCohn condition for the roots of a quadratic polynomial to be inside of the unit disk, namely $|p|<q+1<2$ (see [23]). This inequality for the polynomial $g(t)$ becomes the relation (after some simplification) $\alpha<1+\alpha \gamma-\gamma<\alpha \gamma$, which is true for $\alpha>1, \gamma>1$. Thus (6) is locally asymptotically stable.

The main result of this paper is Theorem 1, which is presented below.

Theorem 1 Assume $\alpha, \gamma$ are arbitrary positive real numbers. Then system (5) has a positive equilibrium $\left(\bar{x}_{+}, \bar{y}_{+}\right)$if and only if $\alpha>1$ and $\gamma>1$. If it exists, the positive equilibrium is unique and given by (6). For arbitrary positive numbers $x_{0}$ and $y_{0}$, let $\left\{\left(x_{n}, y_{n}\right)\right\}$ be given by (5). Then the following statements are true:

(i) If $\alpha<1$, then $\left(x_{n}, y_{n}\right) \rightarrow(0,0)$.

(ii) If $\alpha=1$ and $\gamma<1$, then $y_{n} \rightarrow 0$ and there exists $\bar{x} \geq 0$ such that $\left\{x_{n}\right\}$ is monotonically decreasing and converges to $\bar{x}$.

(iii) If $\alpha=1$ and $\gamma \geq 1$, then $\left(x_{n}, y_{n}\right) \rightarrow(0,0)$.

(iv) If $\alpha>1$ and $\gamma<1$, then $x_{n} \rightarrow \infty$ and $y_{n} \rightarrow 0$.

(v) If $\alpha>1$ and $\gamma=1$, then $x_{n} \rightarrow \infty$ and, for some $\bar{y} \geq 0, y_{n} \rightarrow \bar{y}$.

(vi) If $1<\alpha \leq \gamma$, then $\left(x_{n}, y_{n}\right) \rightarrow\left(\bar{x}_{+}, \bar{y}_{+}\right)$. Furthermore, the positive equilibrium $\left(\bar{x}_{+}, \bar{y}_{+}\right)$is globally asymptotically stable on $(0, \infty) \times(0, \infty)$. 
(vii) If $1<\gamma<\alpha$, the sequence $\left\{\left(x_{n}, y_{n}\right)\right\}$ is bounded and persistent in $(0, \infty) \times$ $(0, \infty)$

The seven dynamical scenarios described in Theorem 1 are depicted in Figure 6, where the various parameter regions are labelled according to the long-term behavior of solutions $\left\{\left(x_{n}, y_{n}\right)\right\}$ of system (5).

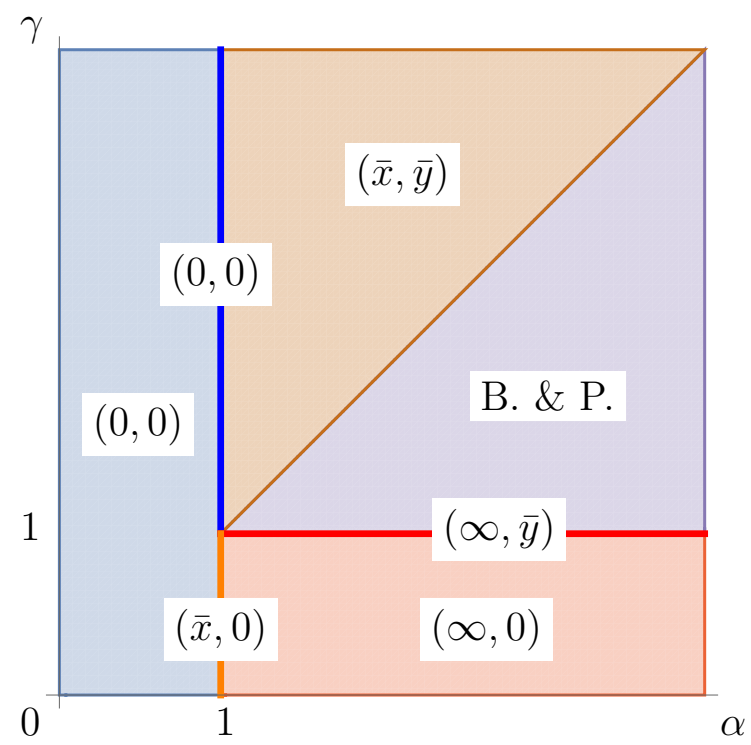

Figure 6: Parameter space regions. Here "B. \& P." stands for bounded and persistent orbits, $(\bar{x}, \bar{y})$ stands for orbits converge to a unique positive equilibrium, $(\bar{x}, 0)$ stands for orbits converge to a point on the $x$-axis, and so on.

Some comments are in order regarding the dynamics of the solutions to system (5) for the parameter region $1<\gamma<\alpha$. Statement (vii) of Theorem 1 states that orbits are bounded and persistent in this case. While this is true, there is also numerical evidence of chaotic behavior for some parameter values in this parameter region. Figures 7 and 8 depict this chaotic behavior for specific choices of $\alpha$ and $\gamma$. It is not known to the authors if chaos is a feature of a substantial portion of the systems associated with the parameter set $1<\gamma<\alpha$. It is important to note that the arguments used to prove the boundedness and persistence of the solutions of system (5) are not affected by the presence of chaotic orbits. 


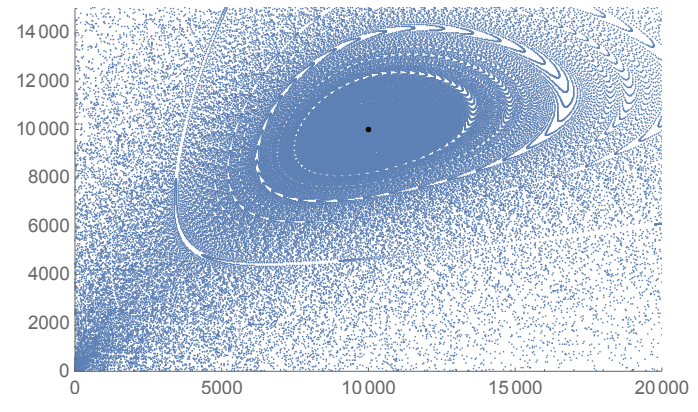

(a)

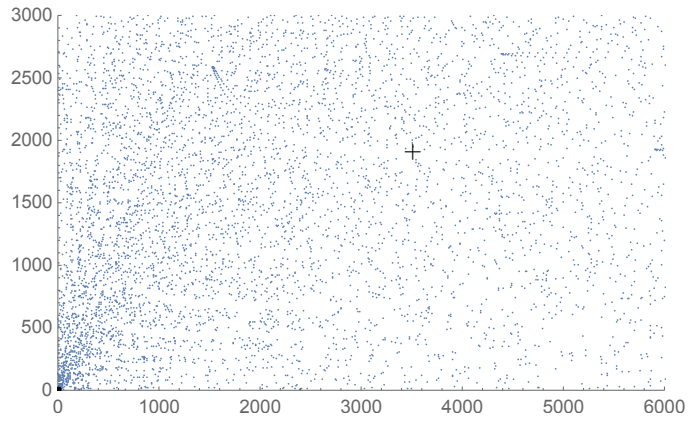

(b)

Figure 7: A plot of the orbit of $(8,8)$, for $\alpha=9999, \gamma=2$. Numerical calculations suggest $\left(x_{n}, y_{n}\right) \rightarrow(\bar{x}, \bar{y})=(9000,9000)$. In plot $(\mathrm{a})$, the equilibrium $(9000,9000)$ is shown as a black dot. Plot (b) shows a smaller window, where some lune-shaped regions apparently devoid of points can be seen. One such region seems to contain the point $(3500,1900)$, which is marked with the symbol +. A total of 300000 points were generated for these plots.

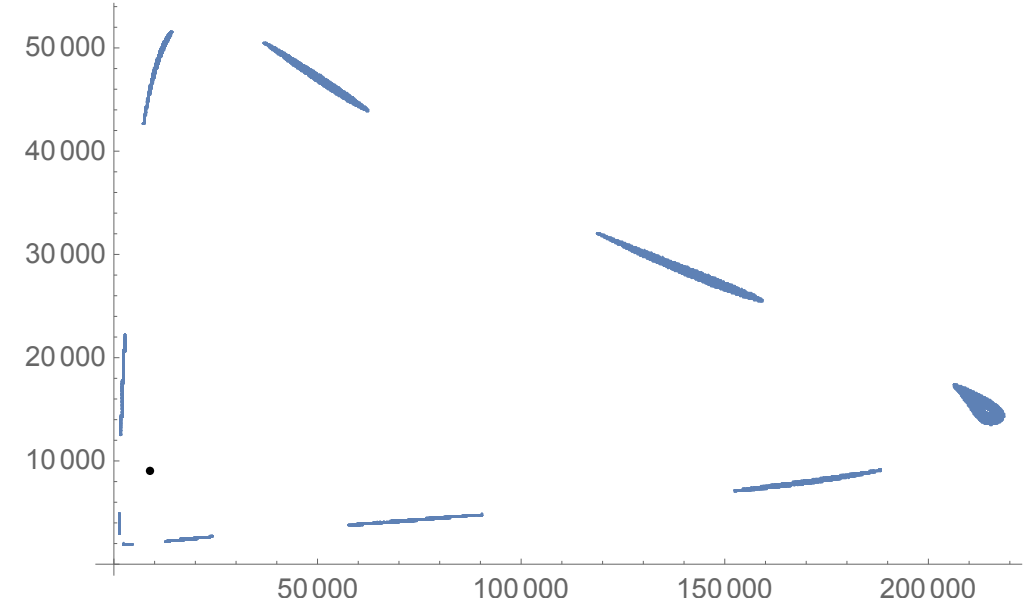

Figure 8: The orbit of $(3500,1900)$, for $\alpha=9999, \gamma=2$ seems to be a subset of 10 connected compact sets, none of which contains the unique positive equilibrium. Compare to Figure 7 . The positive equilibrium $(9000,9000)$ is shown as an isolated dot. A total of 60000 points were generated for this plot. 
The remaining sections of this paper present the proofs for statements (i) through (vii) of Theorem 1. The proofs of statements (i) through (v) regarding the global behavior of solutions to (5) in the absence of a positive equilibrium are done with elementary arguments and are presented in Section 3.2. The proof of statement (vi) regarding the global attractivity of the positive equilibrium is presented in Section 3.3. In this case, we utilize a family $\left\{\mathcal{P}_{\mu}: \mu>0\right\}$ of compact neighborhoods of the positive equilibrium that cover the positive quadrant. In [24], the sets $\mathcal{P}_{\mu}$ are termed balls of the Kobayashi internal metric on the cone (in this case the cone is the nonnegative quadrant). The sets $\mathcal{P}_{\mu}$ are invariant under the map associated with the system and have the property that the $\omega$-limit set is a singleton set, consisting of the positive equilibrium. Thus a proof of global asymptotic stability of the positive equilibrium is obtained when $1<\alpha \leq \gamma$.

A unique positive equilibrium also exists when $1<\gamma<\alpha$, but the sets $\mathcal{P}_{\mu}$ used in part (vi) fail to have desired properties and another approach is needed to establish boundedness and persistence. The proof of statement (vii) regarding the boundedness and persistence of solutions is presented in Section 3.4. We introduce a useful change of coordinates to treat the problem in the whole plane. The subsequent proof is based on the construction of a family of compact sets $\mathcal{K}_{\tau}$. We show that for large enough $\tau$, the sets $\mathcal{K}_{\tau}$ are invariant under the associated map and the collection forms a cover of the plane. Compared to the construction of $\mathcal{P}_{\mu}$, the construction of $\mathcal{K}_{\tau}$ is significantly more involved, as it requires the introduction of auxiliary maps and certain curves that are invariant for those auxiliary maps.

\subsection{Global Behavior in the Absence of a Positive Equilibrium}

This section presents a proof of statements (i) through (v) of Theorem 1. Suppose first that $\alpha \leq 1$. Choose arbitrary positive real numbers $x_{0}$ and $y_{0}$. With 
$\left(x_{n}, y_{n}\right)$ given by $(5)$ for $n>0$, we have

$$
x_{n+1}=\frac{\alpha x_{n}}{1+y_{n}}<\alpha x_{n} \leq x_{n} \text { for all } n \geq 0
$$

and thus

$$
x_{n} \text { converges to a nonnegative real number } \bar{x} \text {. }
$$

Assume $\alpha<1$. If $\bar{x}>0$, then $y_{n} \rightarrow \alpha-1$ by (7), which is impossible for $\alpha<1$. Therefore $x_{n} \rightarrow 0$ as $n \rightarrow \infty$. From (5), $y_{n} \rightarrow 0$ as $n \rightarrow \infty$ and statement (i) follows.

If $\alpha=1$, then there is a continuum of equilibrium points on the extended domain $(0, \infty) \times[0, \infty)$, consisting of points of the form $(x, 0)$ where $x>0$. With $\alpha=1$, we consider three cases: $\gamma<1, \gamma=1$, and $\gamma>1$.

$\alpha=1, \gamma<1$ : From (5), $y_{n+1}<\gamma y_{n}$, thus $\left\{y_{n}\right\}$ is decreasing and convergent to some $\bar{y} \geq 0$. If $\bar{y}>0$, then from (5),

$$
x_{n}=\frac{y_{n+1} y_{n}}{\gamma y_{n}-y_{n+1}} \rightarrow \frac{\bar{y}^{2}}{\gamma \bar{y}-\bar{y}}=\frac{\bar{y}}{\gamma-1} .
$$

The last term in (9) is negative. This implies that $\bar{y}=0$. From this and (8) we conclude $x_{n} \rightarrow \bar{x}>0$ and $y_{n} \rightarrow 0$. Statement (ii) follows.

$\alpha=1, \gamma=1$ : We have $y_{n+1}=\frac{x_{n} y_{n}}{x_{n}+y_{n}}<y_{n}$, therefore $\left\{y_{n}\right\}$ is a decreasing sequence that converges to some $\bar{y} \geq 0$. From $x_{n+1}\left(1+y_{n}\right)=x_{n}$, we have $\bar{x}(1+\bar{y})=$ $\bar{x}$. It follows that if $\bar{x}>0$, then $\bar{y}=0$. But if $\bar{x}=0, y_{n+1}\left(x_{n}+y_{n}\right)=x_{n} y_{n}$ implies $\bar{y}(0+\bar{y})=0 \bar{y}=0$, that is, $\bar{y}=0$. Therefore $\left(x_{n}, y_{n}\right) \rightarrow(\bar{x}, 0)$ for some $\bar{x} \geq 0$. We claim that $\bar{x}=0$. Suppose $\bar{x}>0$. Consider the map $R$ associated with (5) when $\alpha=\gamma=1$ :

$$
R(x, y):=\left(\frac{x}{1+y}, \frac{x y}{x+y}\right), \quad(x, y) \in(0, \infty) \times(0, \infty)
$$

The map $R$ has a real analytic extension $\tilde{R}$ to a neighborhood $\mathcal{N} \subset \mathbb{R}^{2}$ of $(\bar{x}, 0)$. It is shown in [25] that if $\mathcal{N}$ is small enough, then for every point $(x, y) \in \mathcal{N} \backslash\{(\bar{x}, 0)\}$ 
there exists $n>0$ such that $\tilde{R}^{n}(x, y) \notin \mathcal{N}$. This contradicts $x_{n} \rightarrow \bar{x}$, so $\bar{x}=0$. We conclude $x_{n} \rightarrow 0$ and $y_{n} \rightarrow 0$.

$\alpha=1, \gamma>1$ : We claim $\bar{x}=0$. Suppose this is not the case, i.e. $\bar{x}>0$. Then $1+y_{n}=\frac{x_{n}}{x_{n+1}} \rightarrow 1$, so $y_{n} \rightarrow 0$. Also,

$$
\frac{y_{n+1}}{y_{n}}=\frac{\gamma x_{n}}{x_{n}+y_{n}} \rightarrow \gamma>1
$$

which implies $y_{n} \nrightarrow \nrightarrow 0$. This contradicts the assumption, hence $x_{n} \rightarrow 0$. We have,

$$
y_{n+2}=\frac{\gamma x_{n+1} y_{n+1}}{x_{n+1}+y_{n+1}}=\frac{\gamma x_{n} y_{n+1}}{\left(1+y_{n}\right)\left(x_{n+1}+y_{n+1}\right)}<\frac{\gamma x_{n}}{1+y_{n}}<\gamma x_{n}
$$

Therefore, $y_{n} \rightarrow 0$ and statement (iii) follows.

Now, suppose that $\alpha>1$ and $\gamma<1$. Using system (5),

$$
y_{n+1}=\frac{\gamma x_{n} y_{n}}{x_{n}+y_{n}}<\frac{\gamma x_{n} y_{n}}{x_{n}}=\gamma y_{n} \quad \text { for all } n \geq 0
$$

and thus $y_{n} \rightarrow 0$ as $n \rightarrow \infty$. Furthermore, since $\alpha>1$, there exists $N>0$ and $A>1$ such that $\frac{\alpha}{1+y_{n}}>A$ for all $n \geq N$. Then,

$$
x_{n+1}=\frac{\alpha x_{n}}{1+y_{n}}>A x_{n}, \quad n \geq N
$$

Consequently, $x_{n} \rightarrow \infty$ as $n \rightarrow \infty$ and statement (iv) follows.

If $\alpha>1$ and $\gamma=1$, we have

$$
y_{n+1}=\frac{x_{n} y_{n}}{x_{n}+y_{n}}<y_{n} \text { for all } n \geq 0
$$

Thus there exists $\bar{y} \geq 0$ such that $y_{n} \downarrow \bar{y}$. If $\bar{y}=0$, then from $(10), x_{n} \rightarrow \infty$. If $\bar{y}>0$, then from $(5), x_{n}\left(y_{n}-y_{n+1}\right)=y_{n+1} y_{n}$ for $n \geq 0$. As $n \rightarrow \infty, y_{n+1} y_{n} \rightarrow$ $\bar{y}^{2}>0$, which implies $x_{n} \rightarrow \infty$ and statement (v) follows. 


\subsection{Global Attractivity of the Positive Equilibrium}

This section provides a proof of statement (vi) of Theorem 1. Throughout the section we assume that $1<\alpha \leq \gamma$. Under this assumption, there exists a unique positive equilibrium (6) for system (5). Furthermore, the change of variables

$$
x^{\prime}=\left(\frac{\alpha-1}{\gamma-1}\right) \frac{1}{x}, \quad y^{\prime}=(\alpha-1) \frac{1}{y}
$$

conjugates system (5) to

$$
x_{n+1}=a x_{n}+(1-a) \frac{x_{n}}{y_{n}}, \quad y_{n+1}=(1-b) x_{n}+b y_{n}, \quad n=0,1,2, \ldots
$$

where the parameters $a$ and $b$ are

$$
a=\frac{1}{\alpha} \quad \text { and } \quad b=\frac{1}{\gamma}
$$

The map associated with (11) on the positive quadrant is given by

$$
S(x, y)=\left(a x+(1-a) \frac{x}{y},(1-b) x+b y\right), \quad(x, y) \in(0, \infty) \times(0, \infty) .
$$

The assumption $1<\alpha \leq \gamma$ is equivalent to

$$
0<b \leq a<1
$$

in which case the map $S$ has a unique positive fixed point, namely $(1,1)$. We shall prove statement (vi) of Theorem 1 by proving a similar result for (11) under assumption (14). For $\mu>1$, let

$$
\mathcal{P}_{\mu}:=\left\{(x, y): \frac{1}{\mu} \leq x \leq \mu, \frac{1}{\mu} \leq y \leq \mu, \frac{1}{\mu} x \leq y \leq \mu x\right\}
$$

The sets $\mathcal{P}_{2}$ and $\mathcal{P}_{4}$ can be seen in Figure 9 . Note that for each $\mu>1$, the set $\mathcal{P}_{\mu}$ is the convex hull of the points

$$
P_{1}=(\mu, \mu), P_{2}=(1, \mu), P_{3}=\left(\frac{1}{\mu}, 1\right), P_{4}=\left(\frac{1}{\mu}, \frac{1}{\mu}\right), P_{5}=\left(1, \frac{1}{\mu}\right), P_{6}=(\mu, 1) .
$$

Some properties of the sets $\mathcal{P}_{\mu}$ are given in Proposition 1 below. 


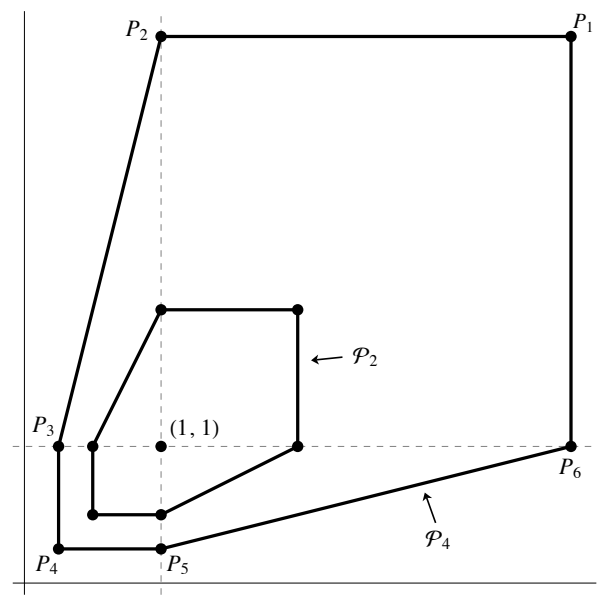

(a)

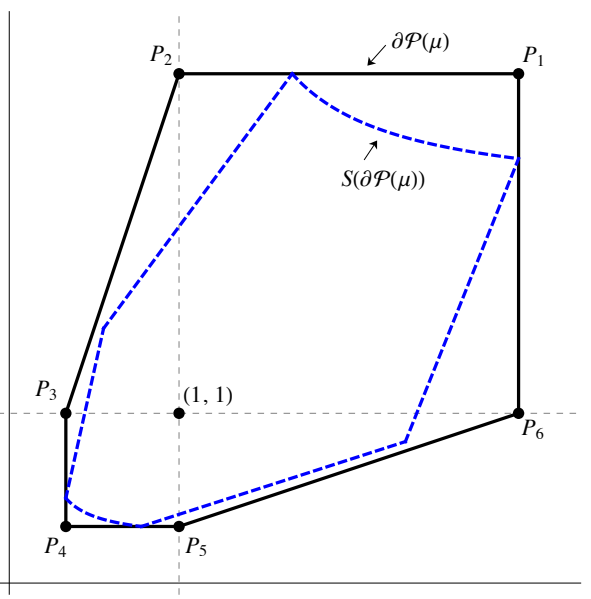

(b)

Figure 9: (a) The sets $\mathcal{P}_{2}$ and $\mathcal{P}_{4}$. (b) The boundary of a set $\mathcal{P}_{\mu}$ (solid) and its image $S\left(\partial \mathcal{P}_{\mu}\right)$ (dashed).

Proposition 1 The following statements are true:

(i) $(1,1) \in \mathcal{P}_{\mu}$ for each $\mu>1$.

(ii) $\cup\left\{P_{\mu}: \mu>1\right\}=(0, \infty) \times(0, \infty)$.

(iii) For every $(x, y) \neq(1,1)$ there exists $\nu>1$ such that $(x, y) \in \partial \mathcal{P}_{\nu}$.

(iv) $S\left(\mathcal{P}_{\mu}\right) \subset \mathcal{P}_{\mu}$ for each $\mu>1$.

(v) $S^{2}\left(\mathcal{P}_{\mu}\right) \subset \operatorname{int}\left(\mathcal{P}_{\mu}\right)$ for each $\mu>1$.

Statements (i) through (iii) of Proposition 1 are obviously true, so here we only prove (iv) and (v). Before we do so, we state a corollary to Proposition 1 that is equivalent to statement (vi) of Theorem 1.

Corollary 3 For every $(x, y) \in(0, \infty) \times(0, \infty), S^{n}(x, y) \rightarrow(1,1)$.

Proof. If $(x, y) \in(0, \infty) \times(0, \infty)$, then (ii) of Proposition 1 implies $(x, y) \in \mathcal{P}_{\mu}$ for some $\mu>1$. By (iv) of Proposition $1, S^{n}(x, y) \in \mathcal{P}_{\mu}$ for all $n \geq 1$. In particular, $\left\{S^{n}(x, y)\right\}$ has at least one accumulation point $(\bar{x}, \bar{y})$. If $\nu>1$ is such 
that $(\bar{x}, \bar{y}) \in \partial \mathcal{P}_{\nu}$, then by continuity of $S$ and by (iv) and (v), $S^{n}(x, y) \in \operatorname{int}\left(\mathcal{P}_{\nu}\right)$ for all $n$ sufficiently large. This is not possible since $(\bar{x}, \bar{y})$ is an accumulation point of $\left\{S^{n}(x, y)\right\}$. Thus $(\bar{x}, \bar{y}) \in \operatorname{int}\left(\mathcal{P}_{\nu}\right)$ for every $\nu>1$, and by (iii), $(\bar{x}, \bar{y})=(1,1)$. Since $(1,1)$ is the only accumulation point of the bounded sequence $\left\{S^{n}(x, y)\right\}$, it follows that $S^{n}(x, y) \rightarrow(1,1)$.

Now, for the proof of (iv) and (v) of Proposition 1 , let $\mu>1$ be fixed but arbitrary, and let $P_{1}, \ldots P_{6}$ be the extreme points or vertices of $\mathcal{P}_{\mu}$ given in (15). We claim first that

$$
S\left(P_{\ell}\right) \in \mathcal{P}_{\mu} \text { for } 1 \leq \ell \leq 6
$$

From (13) and (15),

$$
\begin{array}{lll}
S\left(P_{1}\right)=S(\mu, \mu) & =(1+a(\mu-1), \mu) & \in\left[P_{1}, P_{2}\right], \\
S\left(P_{3}\right)=S\left(\frac{1}{\mu}, 1\right)=\left(\frac{1}{\mu}, \frac{1}{\mu}+b\left(\frac{\mu-1}{\mu}\right)\right) & \in\left[P_{3}, P_{4}\right], \\
S\left(P_{4}\right)=S\left(\frac{1}{\mu}, \frac{1}{\mu}\right)=\left(1+a\left(\frac{1-\mu}{\mu}\right), \frac{1}{\mu}\right) & \in\left[P_{4}, P_{5}\right], \\
S\left(P_{6}\right)=S(\mu, 1)=(\mu, \mu+b(1-\mu)) & \in\left[P_{6}, P_{1}\right] .
\end{array}
$$

Furthermore, $S\left(P_{2}\right)=S(1, \mu)=\left(a+\frac{1-a}{\mu}, 1+b(\mu-1)\right)$ and it can be readily seen that the following inequalities are true:

$\frac{1}{\mu} \leq a+\frac{1-a}{\mu} \leq \mu, \frac{1}{\mu} \leq 1+b(\mu-1) \leq \mu, \frac{1}{\mu}\left(a+\frac{1-a}{\mu}\right) \leq 1+b(\mu-1) \leq \mu\left(a+\frac{1-a}{\mu}\right)$.

That is, $S\left(P_{2}\right) \in \mathcal{P}_{\mu}$. Finally,

$$
S\left(P_{5}\right)=S\left(1, \frac{1}{\mu}\right)=\left(a(1-\mu)+\mu, b\left(\frac{1-\mu}{\mu}\right)+1\right)
$$

and one can similarly conclude that $S\left(P_{5}\right) \in \mathcal{P}_{\mu}$. Thus (16) has been established.

To prove (iv), it is sufficient to prove $S\left(\partial \mathcal{P}_{\mu}\right) \subset \mathcal{P}_{\mu}$. We have

$S\left(\left[P_{1}, P_{2}\right]\right)=\left\{\left(\frac{(a(\mu-1)+1)((\mu-1) t+1)}{\mu}, b(\mu-\mu t+t-1)+(\mu-1) t+1\right): 0 \leq t \leq 1\right\}$.

Hence $S\left(\left[P_{1}, P_{2}\right]\right)$ is a line segment with endpoints in the set $\mathcal{P}_{\mu}$, which is convex. Therefore, $S\left(\left[P_{1}, P_{2}\right]\right) \subset \mathcal{P}_{\mu}$. Similar considerations lead to $S\left(\left[P_{2}, P_{3}\right]\right) \subset \mathcal{P}_{\mu}$, $S\left(\left[P_{4}, P_{5}\right]\right) \subset \mathcal{P}_{\mu}$, and $S\left(\left[P_{5}, P_{6}\right]\right) \subset \mathcal{P}_{\mu}$. 
For $0 \leq t \leq 1$, let $x(t)$ and $y(t)$ be defined by the equation

$(x(t), y(t)):=S\left((1-t) P_{3}+t P_{4}\right)=\left(\frac{\mu+a t-a \mu t}{\mu(\mu+t-\mu t)}, \frac{1+b(-1+\mu+t-\mu t)}{\mu}\right)$.

Then $S\left(\left[P_{3}, P_{4}\right]\right)=\{(x(t), y(t)): 0 \leq t \leq 1\}$, and for $0 \leq t \leq 1$,

$$
\frac{d x}{d t}=\frac{(1-a)(\mu-1)}{(\mu+t-\mu t)^{2}}>0 \quad \text { and } \quad \frac{d y}{d t}=\frac{b(1-\mu)}{\mu}<0 .
$$

Hence, from (16) and (17), $S\left(\left[P_{3}, P_{4}\right]\right) \subset\left[\frac{1}{\mu}, 1\right] \times\left[\frac{1}{\mu}, 1\right]$ and we conclude $S\left(\left[P_{3}, P_{4}\right]\right) \subset$ $\mathcal{P}_{\mu}$. A similar proof (omitted here) yields $S\left(\left[P_{6}, P_{1}\right]\right) \subset \mathcal{P}_{\mu}$. This completes the proof of (iv).

For part (v), from the proof of part (iv), if $(x, y) \in \mathcal{P}_{\mu}$, then $S(x, y) \in \partial \mathcal{P}_{\mu}$ only when $(x, y) \in\left\{P_{1}, P_{3}, P_{4}, P_{6}\right\}$, and otherwise $S(x, y) \in \operatorname{int}\left(\mathcal{P}_{\mu}\right)$ and $S^{2}(x, y) \in$ $\operatorname{int}\left(\mathcal{P}_{\mu}\right)$. In addition, for $1 \leq \ell \leq 6, S\left(P_{\ell}\right) \notin\left\{P_{1}, P_{3}, P_{4}, P_{6}\right\}$, so $S^{2}\left(P_{\ell}\right) \in \operatorname{int}\left(\mathcal{P}_{\mu}\right)$. It follows that $S^{2}\left(\mathcal{P}_{\mu}\right) \subset \operatorname{int}\left(\mathcal{P}_{\mu}\right)$.

\subsection{Boundedness and Persistence of Solutions}

A proof of boundedness and persistence of solutions of system (5) for $1<\gamma<$ $\alpha$ is presented in this section, which corresponds to statement (vii) of Theorem 1.

\subsubsection{Structure of the Proof of Statement (vii) of Theorem 1}

Throughout the section we shall assume the inequality

$$
1<\gamma<\alpha
$$

Under this assumption, there exists a unique positive equilibrium (6) for system (5). The change of variables

$$
x^{\prime}=\left(\frac{\alpha-1}{\gamma-1}\right) \frac{1}{x}, \quad y^{\prime}=\left(\frac{1}{\alpha-1}\right) y
$$

conjugates system (5) to

$$
x_{n+1}=a x_{n}+(1-a) x_{n} y_{n}, \quad y_{n+1}=\frac{y_{n}}{(1-b) x_{n} y_{n}+b}, \quad n=0,1,2, \ldots,
$$


where $a$ and $b$ are as in (12). The map corresponding to (19) is given by

$$
T(x, y)=\left(a x+(1-a) x y, \frac{y}{(1-b) x y+b}\right), \quad(x, y) \in(0, \infty) \times(0, \infty)
$$

Assumption (18) becomes

$$
0<a<b<1
$$

in which case the map $T$ has a unique positive fixed point, namely $(1,1)$. We shall prove (vii) of Theorem 1 by proving a similar statement for (19) under assumption $(21)$.

It is useful to consider logarithmic coordinates. Denote with $L$ and $E$ the planar maps defined for $(x, y) \in(0, \infty) \times(0, \infty)$ and $(u, v) \in \mathbb{R}^{2}$, respectively, by

$$
L(x, y):=(\ln (x), \ln (y)) \text { and } E(u, v):=\left(e^{u}, e^{v}\right)
$$

Set $\hat{T}:=L \circ T \circ E$. That is,

$$
\hat{T}(u, v)=\left(\ln \left(a e^{u}+(1-a) e^{u+v}\right), \ln \left(\frac{e^{v}}{(1-b) e^{u+v}+b}\right)\right), \quad(u, v) \in \mathbb{R}^{2}
$$

Thus $\hat{T}$ is a conjugate of $T$ for which the origin is the (unique) fixed point. An immediate consequence of the definition of $\hat{T}$ is Proposition 2 presented below.

Proposition 2 Let $(x, y)$ be an arbitrary element of $(0, \infty) \times(0, \infty)$. Then the sequence $\left\{T^{n}(x, y)\right\}$ is bounded and persists in $(0, \infty) \times(0, \infty)$ if and only if $\left\{\hat{T}^{n}(L(x, y))\right\}$ is bounded in $\mathbb{R}^{2}$.

It can also be shown that bounded subsets of $\mathbb{R}^{2}$ are contained in $\hat{T}$-invariant compact sets, as described in Proposition 3.

Proposition 3 Suppose $0<a<b<1$. Then for any bounded set $\mathcal{B} \subset \mathbb{R}^{2}$ there exists a $\hat{T}$-invariant compact set $\mathcal{K}$ such that $\mathcal{B} \subset \mathcal{K}$. 
Propositions 2 and 3 have the following corollary, which is precisely statement (vii) of Theorem 1.

Corollary 4 Let $(x, y)$ be an arbitrary element of $(0, \infty) \times(0, \infty)$. Then the sequence $\left\{T^{n}(x, y)\right\}$ is bounded and persists in $(0, \infty) \times(0, \infty)$.

The remainder of this section is devoted to proving Proposition 3. The proof involves constructing a family of compact sets $\mathcal{K}_{\tau}$ that satisfy the properties set forth in Proposition 3 for $\tau$ taken to be sufficiently large. First, we present basic results about $T$ and $\hat{T}$ as well as results related to two auxiliary maps that are useful in the construction of $\mathcal{K}_{\tau}$ and for the arguments that follow.

\subsubsection{Ancillary Properties and Maps}

If $F=\left(f_{1}, f_{2}\right)$ is a map on a planar region $\mathcal{R}$, the equilibrium curves of $F$ are the sets $\left\{(x, y) \in \mathcal{R}: f_{1}(x, y)=x\right\}$ and $\left\{(x, y) \in \mathcal{R}: f_{2}(x, y)=y\right\}$. The equilibrium curves of the maps $T$ and $\hat{T}$ given in (20) and (23) play a prominent role in our proof. Before we go any further, we adopt the following convention in order to simplify notation use:

unless otherwise restricted, $(x, y) \in(0, \infty) \times(0, \infty)$ and $(u, v) \in \mathbb{R}^{2}$.

The equilibrium curves of the maps $T$ are as follows:

$$
\mathcal{C}_{1}:=\{(x, y): y=1\} \quad \text { and } \quad \mathcal{C}_{2}:=\{(x, y): x y=1\}
$$

The equilibrium curves $\mathcal{C}_{1}$ and $\mathcal{C}_{2}$ have $(1,1)$ as their only common point, and the complement in the positive quadrant of their union consists of four disjoint connected components

$$
\begin{array}{ll}
\mathcal{R}_{1}=\{(x, y): y>1 \text { and } x y>1\}, & \mathcal{R}_{2}=\{(x, y): y>1 \text { and } x y<1\} \\
\mathcal{R}_{3}=\{(x, y): y<1 \text { and } x y<1\}, & \mathcal{R}_{4}=\{(x, y): y<1 \text { and } x y>1\}
\end{array}
$$


That is,

$$
(0, \infty) \times(0, \infty) \backslash\left(\mathcal{C}_{1} \cup \mathcal{C}_{2}\right)=\bigcup\left\{\mathcal{R}_{\ell}: 1 \leq \ell \leq 4\right\}
$$

Similarly, the equilibrium curves of the map $\hat{T}$ are

$$
\hat{\mathcal{C}}_{1}:=\{(u, v): v=0\} \quad \text { and } \quad \hat{\mathcal{C}}_{2}:=\{(u, v): u+v=0\}
$$

The curves $\hat{\mathcal{C}}_{1}$ and $\hat{\mathcal{C}}_{2}$ have $(0,0)$ as their only common point, and the complement in the plane of their union consists of four disjoint connected components

$$
\begin{array}{ll}
\hat{\mathcal{R}}_{1}=\{(u, v): v>0 \text { and } u+v>0\}, & \hat{\mathcal{R}}_{2}=\{(u, v): v>0 \text { and } u+v<0\}, \\
\hat{\mathcal{R}}_{3}=\{(u, v): v<0 \text { and } u+v<0\}, & \hat{\mathcal{R}}_{4}=\{(u, v): v<0 \text { and } u+v>0\} .
\end{array}
$$

That is,

$$
\mathbb{R}^{2} \backslash\left(\hat{\mathcal{C}}_{1} \cup \hat{\mathcal{C}}_{2}\right)=\bigcup\left\{\hat{\mathcal{R}}_{\ell}: 1 \leq \ell \leq 4\right\}
$$

The sets $\mathcal{R}_{\ell}$ and $\hat{\mathcal{R}}_{\ell}, 1 \leq \ell \leq 4$, are depicted in Figure 10. Now, denote with $\preceq_{s e}$ the South-East partial order on $\mathbb{R}^{2}$ whose nonnegative cone is the standard fourth quadrant $\{(u, v): u \geq 0, v \leq 0\}$. That is, $\left(u_{1}, v_{1}\right) \preceq_{s e}\left(u_{2}, v_{2}\right)$ if and only if $u_{1} \leq u_{2}$ and $v_{1} \geq v_{2}$. Similarly, denote with $\preceq_{n e}$ the North-East partial order on $\mathbb{R}^{2}$ whose nonnegative cone is the standard first quadrant $\{(u, v): u, v \geq 0\}$. That is, $\left(u_{1}, v_{1}\right) \preceq_{n e}\left(u_{2}, v_{2}\right)$ if and only if $u_{1} \leq u_{2}$ and $v_{1} \leq v_{2}$ (see [26]). Basic monotonicity properties can then be used to prove Proposition 4.

Proposition 4 The following statements are true:

(i) $\quad(x, y) \preceq_{s e} T(x, y)$ for $(x, y) \in \mathcal{R}_{1} \quad$ (i') $\quad(u, v) \preceq_{s e} \hat{T}(u, v)$ for $(u, v) \in \hat{\mathcal{R}}_{1}$

(ii) $\quad(x, y) \preceq_{n e} T(x, y)$ for $(x, y) \in \mathcal{R}_{2} \quad$ (ii') $\quad(u, v) \preceq_{n e} \hat{T}(u, v)$ for $(u, v) \in \hat{\mathcal{R}}_{2}$ (iii) $\quad T(x, y) \preceq_{s e}(x, y)$ for $(x, y) \in \mathcal{R}_{3} \quad$ (iii') $\hat{T}(u, v) \preceq_{s e}(u, v)$ for $(u, v) \in \hat{\mathcal{R}}_{3}$ (iv) $\quad T(x, y) \preceq_{n e}(x, y)$ for $(x, y) \in \mathcal{R}_{4} \quad\left(\right.$ iv' $\left.^{\prime}\right) \quad \hat{T}(u, v) \preceq_{n e}(u, v)$ for $(u, v) \in \hat{\mathcal{R}}_{4}$

We shall need the maps

$$
M(x, y):=\left(a x, \frac{y}{(1-b) x y+b}\right), \quad(x, y) \in(0, \infty) \times(0, \infty),
$$

and

$$
N(x, y):=\left((1-a) x y, \frac{1}{b} y\right), \quad(x, y) \in(0, \infty) \times(0, \infty)
$$



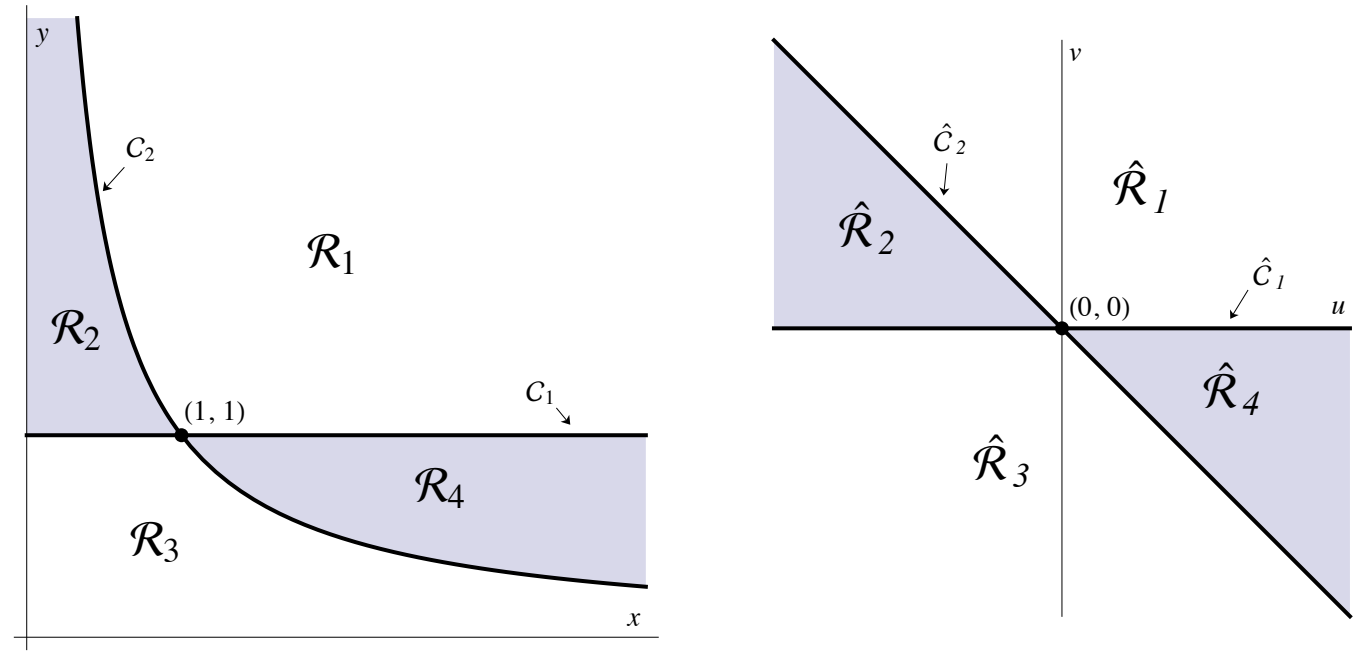

Figure 10: Equilibrium curves and complementary regions for $T$ and $\hat{T}$, respectively.

along with the corresponding conjugate maps $\hat{M}$ and $\hat{N}$ on $\mathbb{R}^{2}$ given in terms of the maps from (22) by

$$
\hat{M}:=L \circ M \circ E \quad \text { and } \quad \hat{N}:=L \circ N \circ E .
$$

To prove the boundedness and persistence of the solutions of system (19), it is important to understand the behavior of the solutions for small values of $x$ and $y$. Close inspection of the map in (20) reveals that $T$ behaves similarly to the map $M$ for values of $y$ close to zero and $T$ behaves similarly to the map $N$ for values of $x$ close to zero. In this way, $M$ and $N$ offer valuable insight into the behavior of solutions of system (19). In Lemmas 1 and 3 that follow, it is proven that there exist invariant curves for the maps $\hat{M}$ and $\hat{N}$ in $\hat{\mathcal{R}}_{2}$ and $\hat{\mathcal{R}}_{3}$, respectively. These curves have important properties when related to the map $\hat{T}$ and play a role in the definition of the family of compact sets $\mathcal{K}_{\tau}$ needed for the proof of Proposition 3. Lemma 2 gives a property of the image of certain line segments in $\hat{\mathcal{R}}_{3}$. This is useful when proving the invariance of the sets $\mathcal{K}_{\tau}$ that are constructed. 
We shall need the constant $r$ given by

$$
r:=\frac{\ln (b)}{\ln (a)}
$$

Under assumption (21), $r$ satisfies

$$
0<r<1
$$

Lemma 1, given below, details an invariant curve corresponding to the map $\hat{M}$ along with properties of its image under $\hat{T}$.

Lemma 1 Let $\tau$ be a fixed but otherwise arbitrary positive real number. Let $\hat{f}_{1}$ : $(-\infty, \tau] \rightarrow \mathbb{R}$ be the function given by

$$
\hat{f}_{1}(u)=-\ln \left(e^{\tau}\left(e^{r(u-\tau)}+\frac{1-b}{b-a}\left(e^{r(u-\tau)}-e^{u-\tau}\right)\right)\right) .
$$

and let $\hat{\mathcal{D}}_{1}$ and $\hat{\mathcal{D}}_{1}^{\prime}$ be the sets

$$
\begin{aligned}
& \hat{\mathcal{D}}_{1}:=\left\{(u, v) \in \mathbb{R}^{2}: v=\hat{f}_{1}(u), u \leq \tau\right\}, \\
& \hat{\mathcal{D}}_{1}^{\prime}:=\left\{(u, v) \in \mathbb{R}^{2}: v=\hat{f}_{1}(u), 0 \leq u \leq \tau, v \leq 0\right\} .
\end{aligned}
$$

Then $\hat{f}_{1}(\cdot)$ is a convex smooth function,

$$
\hat{M}\left(\hat{\mathcal{D}}_{1}\right) \subset \hat{\mathcal{D}}_{1}, \quad \text { and } \quad \hat{T}\left(\hat{\mathcal{D}}_{1}^{\prime}\right) \subset\left\{(u, v) \in \mathbb{R}^{2}: \hat{f}_{1}(u)<v<0, u<\tau\right\} .
$$

Figure 11 shows the curve $\hat{\mathcal{D}}_{1}^{\prime}$ described in Lemma 1 along with its image under the map $\hat{T}$. An extension of $\hat{\mathcal{D}}_{1}^{\prime}$ and its corresponding image in the third quadrant are also included to illustrate the relation $\hat{T}\left(\hat{\mathcal{D}}_{1}^{\prime}\right) \subset$ $\left\{(u, v) \in \mathbb{R}^{2}: \hat{f}_{1}(u)<v<0, u<\tau\right\}$, which is needed in the arguments used in Section 3.4.4.

Proof. A straightforward calculation gives

$$
\hat{f}_{1}^{\prime \prime}(u)=\frac{(1-a)(1-b)(r-1)^{2} e^{-r \tau+r u+\tau+u}}{\left((1-b) e^{u}-(1-a) e^{r(u-\tau)+\tau}\right)^{2}}
$$




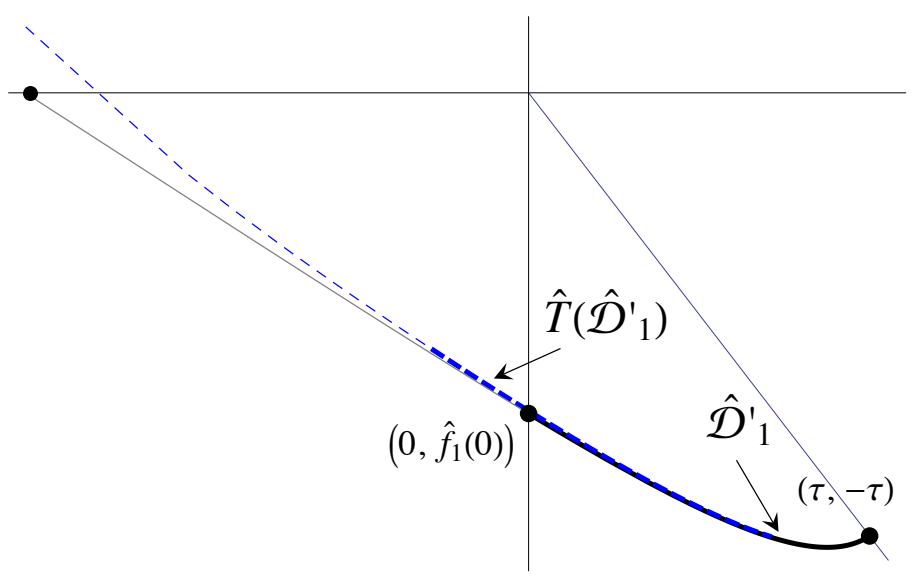

Figure 11: The curve $\hat{\mathcal{D}}_{1}^{\prime}$ (thick, solid) and its image under $\hat{T}$ (thick, dashed) along with an extension of $\hat{\mathcal{D}}_{1}^{\prime}$ (thin, solid) and its image under $\hat{T}$ (thin, dashed).

so $\hat{f}_{1}^{\prime \prime}(u)$ is well defined and positive for $u \leq \tau+\frac{1}{r-1} \ln \left(\frac{1-b}{1-a}\right)$. This inequality, together with (21) and (25), imply that $\hat{f}_{1}^{\prime \prime}(u)$ is defined for $u \leq \tau$, and consequently $v$ is a convex function of $u$ for $u \leq \tau$.

With the change of coordinates $x=e^{u}, y=e^{v}$, together with $x_{0}:=e^{\tau}$ and

$$
\mathcal{D}_{1}:=\left\{(x, y): \frac{1}{x_{0} y}=\left(\frac{x}{x_{0}}\right)^{r}+\frac{1-b}{b-a}\left(\left(\frac{x}{x_{0}}\right)^{r}-\frac{x}{x_{0}}\right), x \leq e^{\tau}\right\}
$$

the inclusion $M\left(\mathcal{D}_{1}\right) \subset \mathcal{D}_{1}$ is equivalent to $\hat{M}\left(\hat{\mathcal{D}}_{1}\right) \subset \hat{\mathcal{D}}_{1}$. We prove the former. Suppose $(x, y) \in \mathcal{D}_{1}$, and set

$$
\left(x^{\prime}, y^{\prime}\right):=M(x, y)=\left(a x, \frac{y}{(1-b) x y+b}\right) .
$$

Then $\left(x^{\prime}, y^{\prime}\right) \in \mathcal{D}_{1}$ if and only if $x^{\prime} \leq e^{\tau}$ and

$$
\frac{(1-b) x y+b}{x_{0} y}=\left(\frac{a x}{x_{0}}\right)^{r}+\frac{1-b}{b-a}\left(\left(\frac{a x}{x_{0}}\right)^{r}-\frac{a x}{x_{0}}\right) .
$$

Through algebraic manipulation, equation (28) may be rewritten as

$$
\frac{b}{x_{0} y}=-(1-b) \frac{x}{x_{0}}+\left(\frac{a x}{x_{0}}\right)^{r}+\frac{1-b}{b-a}\left(\left(\frac{a x}{x_{0}}\right)^{r}-\frac{a x}{x_{0}}\right) .
$$

The equality $a^{r}=b$ and further simplification in (29) give the equation

$$
\frac{1}{x_{0} y}=\left(\frac{x}{x_{0}}\right)^{r}+\frac{1-b}{b-a}\left(\frac{x}{x_{0}}\right)^{r}-\frac{1-b}{b-a}\left(\frac{x}{x_{0}}\right) \text {. }
$$


Since by assumption $(x, y) \in \mathcal{D}_{1}$, we have (30) is true. It is also the case that $x^{\prime}=a x \leq a e^{\tau}<e^{\tau}$. This proves $\left(x^{\prime}, y^{\prime}\right) \in \mathcal{D}_{1}$.

To prove the second inclusion in $(27)$, consider $(u, v) \in \hat{\mathcal{D}}_{1}^{\prime}$, and set $\left(u^{\prime}, v^{\prime}\right)=$ $\hat{M}(u, v)$ and $\left(u^{\prime \prime}, v^{\prime \prime}\right)=\hat{T}(u, v)$. Thus $\left(u^{\prime}, v^{\prime}\right) \in \hat{\mathcal{D}}_{1}$. From the definition of $\hat{M}$ and $\hat{T}$ we have

$$
u^{\prime}<u^{\prime \prime} \text { and } \quad v^{\prime}=v^{\prime \prime} \text {. }
$$

Consider the function $\psi(t)$ with $t \leq \tau$, given by

$$
\psi(t)=e^{r(t-\tau)}+\frac{1-b}{b-a}\left(e^{r(t-\tau)}-e^{t-\tau}\right) .
$$

Since $\left(u^{\prime}, v^{\prime}\right) \in \hat{\mathcal{D}}_{1}$ then $v^{\prime}=\hat{f}_{1}\left(u^{\prime}\right)=-\ln \left(e^{\tau} \psi\left(u^{\prime}\right)\right)$. Therefore, $e^{-\tau-v^{\prime}}=\psi\left(u^{\prime}\right)$. This fact, (31), and the increasing character of $\psi$ give

$$
e^{-\tau-v^{\prime \prime}}=e^{-\tau-v^{\prime}}=\psi\left(u^{\prime}\right)<\psi\left(u^{\prime \prime}\right)
$$

Inequality $(32)$ implies $\hat{f}_{1}\left(u^{\prime \prime}\right)<v^{\prime \prime}$, which together with

$$
v^{\prime \prime}=\ln \left(\frac{e^{v}}{(1-b) e^{u+v}+b}\right)<0
$$

complete the proof of the second inclusion in (27). See Figure 11.

Lemma 2, given below, details a property of the image under $\hat{T}$ of certain line segments.

Lemma 2 Let $p$ and $q$ be arbitrary negative numbers such that $\frac{q}{p}<r$, where $r$ is defined in (24). Let $\hat{\mathcal{D}}_{2}$ be the line in the plane through $(p, 0)$ and $(0, q)$, and let $\hat{\mathcal{D}}_{2}^{\prime}$ be the line segment whose endpoints are $(p, 0)$ and $(0, q)$. Then $\hat{T}\left(\hat{\mathcal{D}}_{2}^{\prime}\right)$ is a subset of the connected component of $\mathbb{R}^{2} \backslash \hat{\mathcal{D}}_{2}$ that contains the origin.

Proof. For $u \leq 0, v<0$, consider the real valued function

$$
\phi(u, v):=-\frac{\ln \left((1-b) e^{u+v}+b\right)}{\ln \left(a+(1-a) e^{v}\right)} .
$$




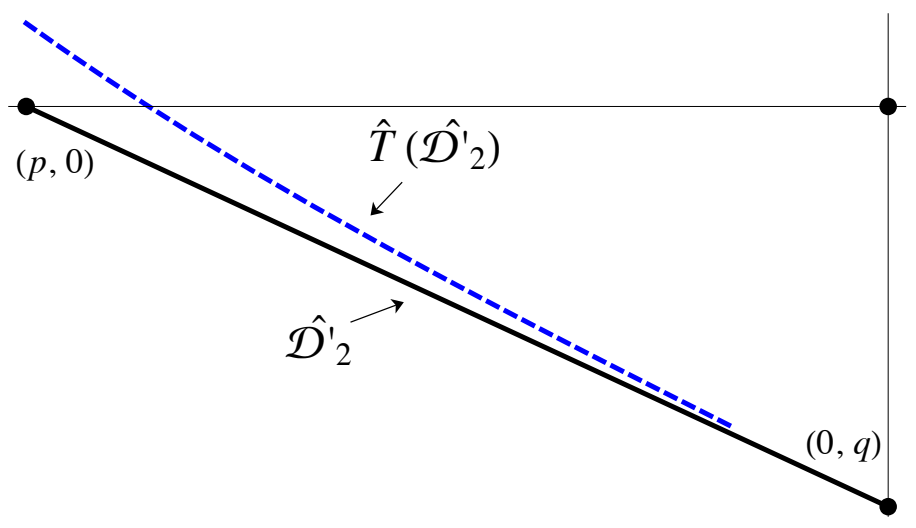

Figure 12: The curve $\hat{\mathcal{D}}_{2}^{\prime}$ (solid) and its image under $\hat{T}$ (dashed)

We claim

$$
\phi(u, v) \leq-\frac{\ln (b)}{\ln (a)}=-r, \quad u \leq 0, v<0 .
$$

It can be easily shown that for fixed $v \leq 0, \phi(u, v)$ is increasing in $u$. Therefore, it is sufficient to verify (33) for $\phi(0, v)$. Equivalently, with $y:=e^{v}$, we will verify that $f(y) \leq-r$ for all $y \in(0,1)$, where

$$
f(y)=-\frac{\ln ((1-b) y+b)}{\ln (a+(1-a) y)} .
$$

Notice,

$$
t+\ln (1-t)<0 \quad \text { for } \quad t \in(0,1) .
$$

Therefore, for $r \in(0,1)$ and $y \in(0,1)$,

$$
\begin{aligned}
& \frac{\partial}{\partial r}\left[\frac{\left(\left(1-a^{r}\right) y+a^{r}\right) \ln \left(\left(1-a^{r}\right) y+a^{r}\right)}{1-a^{r}}\right] \\
& =\frac{a^{r} \ln (a)\left(\left(1-a^{r}\right)(1-y)+\ln \left(1-\left(1-a^{r}\right)(1-y)\right)\right)}{\left(1-a^{r}\right)^{2}}>0,
\end{aligned}
$$

where (34) was used with $t=\left(1-a^{r}\right)(1-y)$ to conclude (35). The inequality in (35), along with $b=a^{r}$ from (24), imply

$$
\begin{aligned}
\frac{((1-b) y+b) \ln ((1-b) y+b)}{1-b} & =\frac{\left(\left(1-a^{r}\right) y+a^{r}\right) \ln \left(\left(1-a^{r}\right) y+a^{r}\right)}{1-a^{r}} \\
& <\frac{((1-a) y+a) \ln ((1-a) y+a)}{1-a} .
\end{aligned}
$$


It follows from (36) that for all $y \in(0,1)$,

$$
\frac{d}{d y}[f(y)]=\frac{\frac{(1-a) \ln ((1-b) y+b)}{(1-a) y+a}-\frac{(1-b) \ln ((1-a) y+a)}{(1-b) y+b}}{(\ln ((1-a) y+a))^{2}}<0 .
$$

Consequently, $f(y) \leq f(0)=-\frac{\ln (b)}{\ln (a)}=-r$, and statement (33) is established. Now, assume $(u, v) \in \hat{\mathcal{D}}_{2}^{\prime}$. Since

$$
\hat{T}(u, v)-(u, v)=\left(\ln \left(a+(1-a) e^{v}\right),-\ln \left((1-b) e^{u+v}+b\right)\right)
$$

the slope of the line through $(u, v)$ and $\hat{T}(u, v)$ is precisely $\phi(u, v)$. By Proposition 4, $T(u, v) \preceq_{s e}(u, v)$. From the latter relation, (33), (37), and the hypothesis on the slope of $\hat{\mathcal{D}}_{2}$, namely $-\frac{q}{p}$ being greater than $-r$, it follows that $\hat{T}(u, v)$ and $(0,0)$ belong to the same component of $\mathbb{R}^{2} \backslash \hat{\mathcal{D}}_{2}$. The curve $\hat{\mathcal{D}}_{2}^{\prime}$ and its image under $\hat{T}$ can be seen in Figure 12 .

The final lemma in this subsection details an invariant curve corresponding to the map $\hat{N}$ along with properties of its image under $\hat{T}$. Prior to stating the lemma, we verify that

$$
\hat{T}(\{(u, v): u+v \geq 0, u \leq 0, v>0\}) \subset \hat{\mathcal{R}}_{1} .
$$

Consider $(u, v) \in\{(s, t): s+t \geq 0, s \leq 0, t>0\}$ such that $u+v=0$ and notice

$$
\hat{T}(u, v)=\left(\ln \left(a e^{u}+(1-a)\right), v\right)
$$

Since $u<0$ implies $\ln \left(a e^{u}+(1-a)\right)>u$, it follows from $(39)$ that $\hat{T}(u, v) \in \hat{\mathcal{R}}_{1}$. Similarly, consider $(u, v) \in\{(s, t): s+t \geq 0, s \leq 0, t>0\}$ such that $u=0$ and notice

$$
\hat{T}(u, v)=\left(\ln \left(a+(1-a) e^{v}\right), \ln \left(\frac{e^{v}}{(1-b) e^{v}+b}\right)\right) .
$$

Since $v>0, \hat{T}(u, v)$ is in the first quadrant of the plane and thus belongs to $\hat{\mathcal{R}}_{1}$. By continuity of $\hat{T},(38)$ follows. This relation will be helpful in proving Lemma 3 below. 
Lemma 3 Let $c_{0}$ be a fixed but otherwise arbitrary negative real number, and set

$$
c_{1}:=-\frac{1}{2}-\frac{\ln (1-a)}{\ln (b)} \quad \text { and } \quad c_{2}:=-\frac{1}{2 \ln (b)} .
$$

Let $\hat{\mathcal{D}}_{3}$ and $\hat{\mathcal{D}}_{3}^{\prime}$ be the sets

$$
\begin{aligned}
& \hat{\mathcal{D}}_{3}:=\left\{(u, v) \in \mathbb{R}^{2}: u=c_{2} v^{2}+c_{1} v+c_{0}\right\}, \\
& \hat{\mathcal{D}}_{3}^{\prime}:=\hat{\mathcal{D}}_{3} \cap\{(u, v): u \leq 0, v \geq 0\} .
\end{aligned}
$$

Then,

$$
\hat{N}\left(\hat{\mathcal{D}}_{3}\right) \subset \hat{\mathcal{D}}_{3} \quad \text { and } \quad \hat{T}\left(\hat{\mathcal{D}}_{3}^{\prime}\right) \subset\left\{(u, v): u>c_{2} v^{2}+c_{1} v+c_{0}, v>0\right\}
$$

Figure 13 shows the curve $\hat{\mathcal{D}}_{3}^{\prime}$ described in Lemma 3 along with its image under the map $\hat{T}$ and illustrates the relation $\hat{T}\left(\hat{\mathcal{D}}_{3}^{\prime}\right) \subset\left\{(u, v): u>c_{2} v^{2}+c_{1} v+c_{0}, v>\right.$ $0\}$.

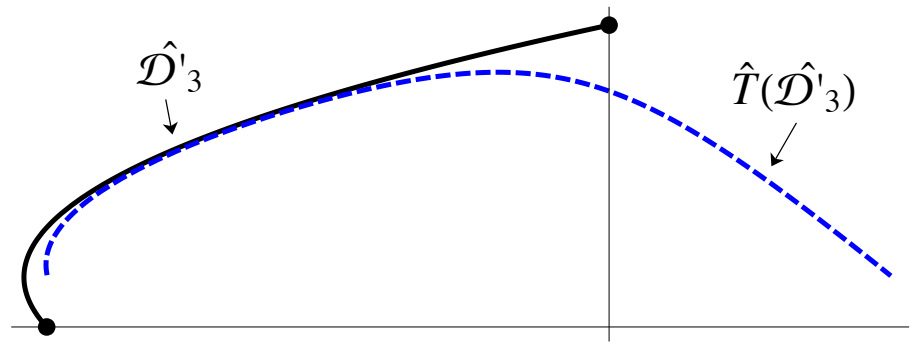

Figure 13: The curve $\hat{\mathcal{D}}_{3}^{\prime}$ (solid) and its image under $\hat{T}$ (dashed)

Proof. Let $(u, v) \in \hat{\mathcal{D}}_{3}$ (i.e. $u=c_{2} v^{2}+c_{1} v+c_{0}$ ) and set

$$
\left(u^{\prime}, v^{\prime}\right):=\hat{N}(u, v)=\left(\ln \left((1-a) e^{u+v}\right), \ln \left(\frac{1}{b} e^{v}\right)\right) .
$$

Then,

$$
\begin{aligned}
c_{2}\left(v^{\prime}\right)^{2} & +c_{1} v^{\prime}+c_{0} \\
& =c_{2}(v-\ln (b))^{2}+c_{1}(v-\ln (b))+c_{0} \\
& =c_{2} v^{2}-2 c_{2}(\ln (b)) v+c_{2}(\ln (b))^{2}+c_{1} v-c_{1} \ln (b)+c_{0} \\
& =u-2 c_{2}(\ln (b)) v+c_{2}(\ln (b))^{2}-c_{1} \ln (b) .
\end{aligned}
$$


A straightforward calculation using (40) gives

$$
-2 c_{2}(\ln (b))=1 \quad \text { and } \quad c_{2}(\ln (b))^{2}-c_{1} \ln (b)=\ln (1-a) .
$$

Consequently, from (42), (43) and (44) we have

$$
c_{2}\left(v^{\prime}\right)^{2}+c_{1} v^{\prime}+c_{0}=u+v+\ln (1-a)=\ln \left((1-a) e^{u+v}\right)=u^{\prime} .
$$

This proves the first relation in (41). To prove the second relation in (41), let $(u, v) \in \hat{\mathcal{D}}_{3}^{\prime}$. Since $0 \leq v \leq q_{2}$ and $u \leq 0$,

$$
(1-b) e^{u+v}+b<\max \left\{e^{u+v}, 1\right\} \leq \max \left\{e^{v}, 1\right\}=e^{v}
$$

and it follows that

$$
\ln \left(\frac{e^{v}}{(1-b) e^{u+v}+b}\right)>0
$$

Consequently, $\hat{T}(u, v) \in\{(s, t): t>0\}$. Now, define

$$
\mathcal{Q}_{-}:=\left\{(u, v) \in \hat{\mathcal{D}}_{3}^{\prime}: u+v \leq 0\right\} \quad \text { and } \quad \mathcal{Q}_{+}:=\left\{(u, v) \in \hat{\mathcal{D}}_{3}^{\prime}: u+v>0\right\}
$$

Clearly, $\hat{\mathcal{D}}_{3}^{\prime}=\mathcal{Q}_{-} \cup \mathcal{Q}_{+}$. We consider two cases separately. If $(u, v) \in \mathcal{Q}_{+}$, then by Proposition 4 we have $(u, v) \preceq_{s e} \hat{T}(u, v)$. Combining this with (38), it follows that $\hat{T}(u, v) \in\left\{(s, t): s>c_{2} t^{2}+c_{1} t+c_{0}, t>0\right\}$. If now $(u, v) \in \mathcal{Q}_{-}$, then by Proposition $4,(u, v) \preceq_{n e} \hat{T}(u, v)$. Also, note that for $x:=e^{u}$ and $y:=e^{v}$,

$$
T(x, y)-N(x, y)=\left(a x,-\frac{(1-b) x y}{b((1-b) x y+b)}\right) .
$$

Hence $N(x, y) \preceq_{s e} T(x, y)$, which implies $\hat{N}(u, v) \preceq_{s e} \hat{T}(u, v)$. Now, $\hat{N}(u, v) \in \hat{\mathcal{D}}_{3}$ by the first part of this proof and the relation $\hat{T}(u, v) \in\left\{(s, t): s>c_{2} t^{2}+c_{1} t+\right.$ $\left.c_{0}, t>0\right\}$ follows. The curve $\hat{\mathcal{D}}_{3}^{\prime}$ along with its image under $\hat{T}$ can be seen in Figure 13. 


\subsubsection{Construction of a Family of Compact Sets}

We begin by establishing some useful inequalities. We shall need the following values, which can be obtained from equation (26):

$$
\hat{f}_{1}(0)=\ln \left(\frac{(b-a) e^{\tau(r-1)}}{(b-a)+(1-b)\left(1-e^{\tau(r-1)}\right)}\right)
$$

and

$$
\hat{f}_{1}^{\prime}(0)=-\frac{1-b-(1-a) r e^{\tau(1-r)}}{1-b-(1-a) e^{\tau(1-r)}}
$$

Lemma 4, presented below, is easily established from relations (21), (25), (46) and (47).

Lemma 4 There exists $\tau_{1}>0$ such that

$$
\hat{f}_{1}(0)<0, \quad \text { for } \quad \tau \geq \tau_{1}
$$

and

$$
\hat{f}_{1}^{\prime}(0)<0, \quad \text { for } \quad \tau \geq \tau_{1}
$$

The sets $\mathcal{K}_{\tau}$ are introduced next.

Definition 7 Let $\tau \in \mathbb{R}_{+}$be such that $\tau \geq \tau_{1}$ with $\tau_{1}$ as in Lemma 4, and set

$$
\begin{aligned}
& q_{1}:=\hat{f}_{1}(0), \\
& p_{2}:=-\frac{\hat{f}_{1}(0)}{\hat{f}_{1}^{\prime}(0)}, \quad \text { and } \\
& q_{2}:=\frac{-c_{1}+\sqrt{c_{1}^{2}-4 c_{2} p_{2}}}{2 c_{2}},
\end{aligned}
$$

where $\hat{f}_{1}(0), \hat{f}_{1}^{\prime}(0), c_{1}$ and $c_{2}$ are given in (40), (46), and (47). Let the set $\mathcal{K}_{\tau}$ be the convex hull of the sets $\hat{\mathcal{D}}_{\ell}^{\prime}, 0 \leq \ell \leq 4$, where

$\hat{\mathcal{D}}_{0}^{\prime} \quad$ is the line segment joining $(\tau, 0)$ and $(\tau,-\tau)$.

$\hat{\mathcal{D}}_{1}^{\prime} \quad$ is the curve given in Lemma 1 with endpoints $(\tau,-\tau)$ and $\left(0, q_{1}\right)$.

$\hat{\mathcal{D}}_{2}^{\prime} \quad$ is the line segment with endpoints $\left(0, q_{1}\right)$ and $\left(p_{2}, 0\right)$.

$\hat{\mathcal{D}}_{3}^{\prime} \quad$ is the parabolic arch in Lemma 3 with endpoints at $\left(p_{2}, 0\right)$ and $\left(0, q_{2}\right)$.

$\hat{\mathcal{D}}_{4}^{\prime} \quad$ is the line segment with endpoints $\left(0, q_{2}\right)$ and $(\tau, 0)$. 
Remark 1 In Definition 7, $q_{1}<0$ and $p_{2}<0$ by Lemma 4 . Therefore, $\mathcal{K}_{\tau}$ is a compact and convex neighborhood of the origin such that $\partial \mathcal{K}_{\tau}=\cup_{\ell=0}^{4} \hat{\mathcal{D}}_{\ell}^{\prime}$. See Figure 14 .

Remark 2 In order to simplify notation, dependence on $\tau$ has been suppressed in the terms $q_{1}, p_{1}, q_{2}$, and $\hat{\mathcal{D}}_{\ell}^{\prime}, 0 \leq \ell \leq 4$.

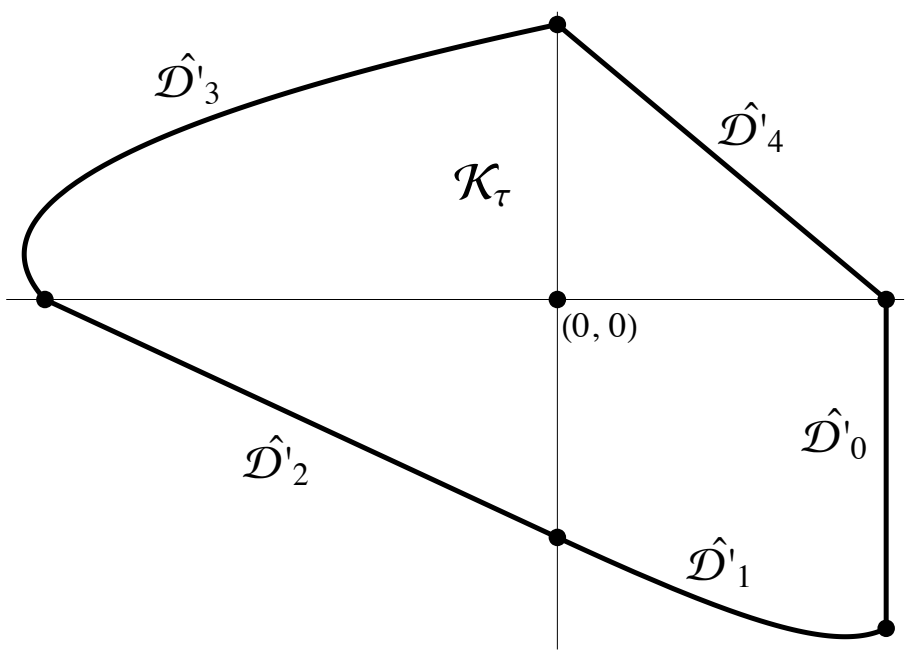

Figure 14: A set $\mathcal{K}_{\tau}$ whose boundary consists of the sets $\hat{\mathcal{D}}_{\ell}^{\prime}$ for $0 \leq \ell \leq 4$

The proof of Proposition 3 involves an asymptotic argument on the parameter $\tau$ as it relates to the compact set $\mathcal{K}_{\tau}$. It is useful for us to first describe the asymptotic behavior of $q_{1}, q_{2}$, and $p_{2}$ when $\tau \rightarrow+\infty$.

Claim 1 The asymptotic behavior of $q_{1}, q_{2}$, and $p_{2}$ is as follows:

(i) $q_{1}=(r-1) \tau+\mathcal{O}(1)$ as $\tau \rightarrow+\infty$.

(ii) $p_{2}=\left(\frac{r-1}{r}\right) \tau+\mathcal{O}(1)$ as $\tau \rightarrow+\infty$.

(iii) $q_{2}=\sqrt{2 \ln (b) \frac{r-1}{r}} \sqrt{\tau}+\mathcal{O}(1)$ as $\tau \rightarrow+\infty$. 
Proof. From (46) and (48),

$$
\begin{aligned}
q_{1} & =(r-1) \tau+\ln (b-a)-\ln \left((b-a)+(1-b)\left(1-e^{\tau(r-1)}\right)\right) \\
& =(r-1) \tau+\ln \left(\frac{1}{1+\frac{1-b}{b-a}\left(1-e^{\tau(r-1)}\right)}\right) .
\end{aligned}
$$

Since $r \in(0,1)$, (i) follows. Similarly,

$$
\begin{aligned}
p_{2}=-\frac{\hat{f}_{1}(0)}{\hat{f}_{1}^{\prime}(0)} & =-\frac{1}{\hat{f}_{1}^{\prime}(0)} \ln \left(\frac{(b-a) e^{\tau(r-1)}}{(b-a)+(1-b)\left(1-e^{\tau(r-1)}\right)}\right) \\
& =\frac{(1-r)}{\hat{f}_{1}^{\prime}(0)} \tau-\frac{\ln (b-a)}{\hat{f}_{1}^{\prime}(0)}+\frac{\ln \left((b-a)+(1-b)\left(1-e^{\tau(r-1)}\right)\right)}{\hat{f}_{1}^{\prime}(0)} .
\end{aligned}
$$

Since $r \in(0,1)$ and $\lim _{\tau \rightarrow \infty} \hat{f}_{1}^{\prime}(0)=-r$, (ii) follows. Finally, from (49) and (ii),

$$
\begin{aligned}
q_{2} & =\frac{-c_{1}+\sqrt{c_{1}^{2}-4 c_{2} p_{2}}}{2 c_{2}} \\
& =\sqrt{-\frac{p_{2}}{c_{2}}+\mathcal{O}(1)} \\
& =\sqrt{-\left(\frac{r-1}{c_{2} r}\right) \tau}+\mathcal{O}(1),
\end{aligned}
$$

and thus (iii) follows from substituting $c_{2}=-1 /(2 \ln (b))$.

\subsubsection{Proof of Proposition 3}

To prove Proposition 3, we establish first that any given bounded set $\mathcal{B} \subset \mathbb{R}^{2}$ is contained in $\mathcal{K}_{\tau}$ for $\tau$ large enough.

Claim 2 Let $\mathcal{B} \subset \mathbb{R}^{2}$ be bounded. Then for all $\tau$ large enough, $\mathcal{B} \subset \mathcal{K}_{\tau}$.

Proof. Since $\mathcal{K}_{\tau}$ is convex, the quadrilateral $\mathcal{S}$ whose endpoints are $(\tau, 0),\left(0, q_{1}\right)$, $\left(p_{2}, 0\right)$ and $\left(0, q_{2}\right)$ is such that $\mathcal{S} \subset \mathcal{K}_{\tau}$ (see Figure 15). Therefore, Claim 1 implies that for all large enough $\tau, \mathcal{K}_{\tau}$ contains $\mathcal{B}$.

Next we prove that for all $\tau$ large enough, $\hat{T}\left(\hat{\mathcal{D}}_{\ell}^{\prime}\right) \subset \mathcal{K}_{\tau}$ for $0 \leq \ell \leq 4$. Once this has been established, it follows that $\mathcal{K}_{\tau}$ is $\hat{T}$-invariant for large $\tau$ and the proof 


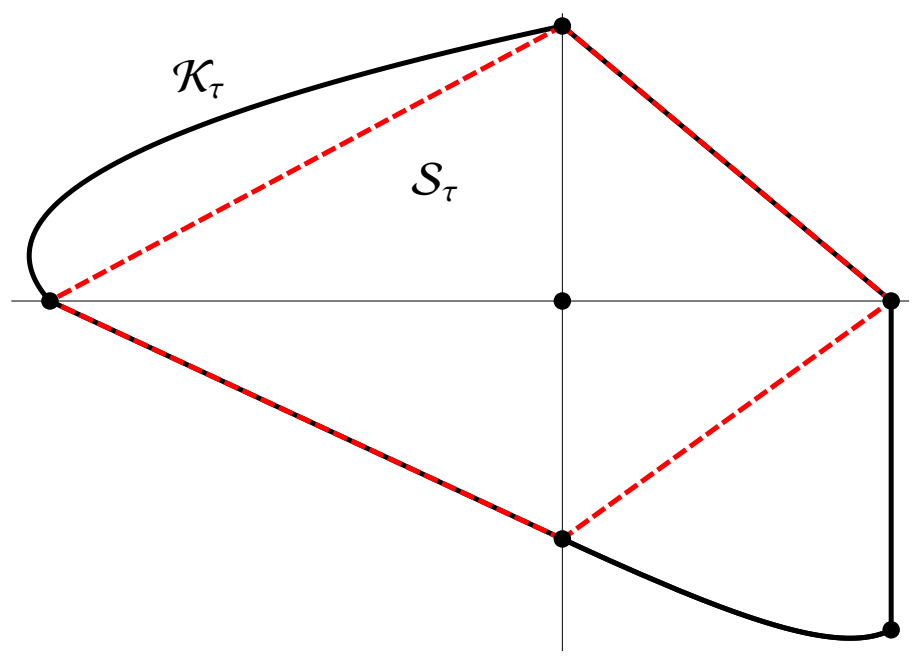

Figure 15: The quadrilateral $\mathcal{S}_{\tau}$ with $\mathcal{S}_{\tau} \subset \mathcal{K}_{\tau}$

of Proposition 3 will be complete. The boundary of $\mathcal{K}_{\tau}$ along with its image under the map $\hat{T}$ can be seen in Figure 16. We assume in Claims 3 through 7 that $\tau \geq \tau_{1}$.

Claim $3 \hat{T}\left(\hat{\mathcal{D}}_{0}^{\prime}\right) \subset \mathcal{K}_{\tau}$.

Proof. Let us first verify that the endpoints of $\hat{T}\left(\hat{\mathcal{D}}_{0}^{\prime}\right)$, namely the points $\hat{T}(\tau, 0)$ and $\hat{T}(\tau,-\tau)$, belong to $\mathcal{K}_{\tau}$. Notice $\hat{T}(\tau, 0)=\left(\tau,-\ln \left((1-b) e^{\tau}+b\right)\right)$ satisfies $-\ln \left((1-b) e^{\tau}+b\right)>-\tau$, hence $\hat{T}(\tau, 0) \in \mathcal{K}_{\tau}$. Also, $\hat{T}(\tau,-\tau)=$ $\left(\ln \left(a e^{\tau}+(1-a)\right),-\tau\right)$ satisfies $0<\ln \left(a e^{\tau}+(1-a)\right)<\tau$. Since $(\tau,-\tau) \in \hat{\mathcal{D}}_{1}^{\prime}$, it follows from Lemma 1 that $\hat{T}(\tau,-\tau) \in \mathcal{K}_{\tau}$, so both endpoints of $\hat{T}\left(\hat{\mathcal{D}}_{0}^{\prime}\right)$ belong to $\mathcal{K}_{\tau}$.

We now show that $\hat{T}\left(\hat{\mathcal{D}}_{0}^{\prime}\right)$ is a curve linearly ordered in the $\preceq_{n e}$ partial order. We may write $\hat{\mathcal{D}}_{0}^{\prime}=\{(\tau,-\tau t): 0 \leq t \leq 1\}$. For $0 \leq t \leq 1$ set

$$
(\tilde{u}(t), \tilde{v}(t)):=\hat{T}((\tau,-\tau t))=\left(\ln \left(a e^{\tau}+(1-a) e^{\tau(1-t)}\right), \ln \left(\frac{e^{-\tau t}}{(1-b) e^{\tau(1-t)}+b}\right)\right) .
$$

Then $\hat{T}\left(\hat{\mathcal{D}}_{0}^{\prime}\right)=\{(\tilde{u}(t), \tilde{v}(t)): 0 \leq t \leq 1\}$. From $(50)$,

$$
\frac{d \tilde{u}}{d t}=-\frac{(1-a) \tau e^{\tau(1-t)}}{(1-a) e^{\tau(1-t)}+a e^{\tau}}<0 \quad \text { and } \quad \frac{d \tilde{v}}{d t}=-\frac{b \tau e^{\tau t}}{(1-b) e^{\tau}+b e^{\tau t}}<0 .
$$


Thus both $\tilde{u}(t)$ and $\tilde{v}(t)$ are decreasing functions of $t$ in $[0,1]$, so $\hat{T}\left(\hat{\mathcal{D}}_{0}^{\prime}\right)$ is a curve linearly ordered in the $\preceq_{n e}$ partial order. It follows that $\hat{T}\left(\hat{\mathcal{D}}_{0}^{\prime}\right)$ is a subset of the closed rectangular region $\mathcal{R}$ determined by the vertices $\hat{T}(\tau, 0)$ and $\hat{T}(\tau,-\tau)$. Since the second coordinate of $\hat{T}(\tau,-\tau)$ is equal to $-\tau$ and $\hat{\mathcal{D}}_{1}^{\prime}$ is the graph of a convex function, it follows from $(27)$ that $\mathcal{R} \subset \mathcal{K}_{\tau}$, and consequently, $\hat{T}\left(\hat{\mathcal{D}}_{0}^{\prime}\right) \subset \mathcal{K}_{\tau}$.

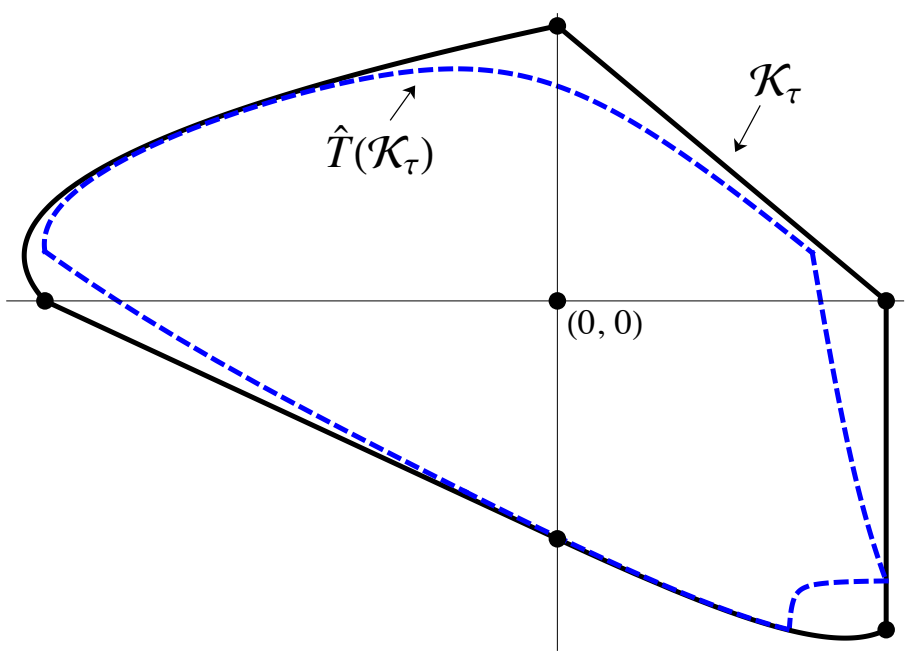

Figure 16: Boundary of the set $\mathcal{K}_{\tau}$ (solid) and its image under $\hat{T}$ (dashed).

Claim $4 \hat{T}\left(\hat{\mathcal{D}}_{1}^{\prime}\right) \subset \mathcal{K}_{\tau}$.

Proof. For $(u, v) \in \hat{\mathcal{D}}_{1}^{\prime}$ arbitrary but fixed, let $(\tilde{u}, \tilde{v})$ be given by

$$
(\tilde{u}, \tilde{v})=\hat{T}(u, v)=\left(\ln \left(a e^{u}+(1-a) e^{u+v}\right), \ln \left(\frac{e^{v}}{(1-b) e^{u+v}+b}\right)\right) .
$$

By the second relation in (27) of Lemma 1 , and convexity of $\mathcal{K}_{\tau}$ and $\hat{\mathcal{D}}_{1}$, it is sufficient to verify that $\tilde{v}<0$. Notice $(u, v) \in \hat{\mathcal{D}}_{1}^{\prime}$ implies $u>0, v<0$, and $(1-b) e^{u+v}+b>(1-b) e^{v}+b>e^{v}$. It follows that $\tilde{v}<0$. 
Claim $5 \hat{T}\left(\hat{\mathcal{D}}_{2}^{\prime}\right) \subset \mathcal{K}_{\tau}$.

Proof. The line segment $\hat{\mathcal{D}}_{2}^{\prime}$ has slope $-\frac{q_{1}}{p_{2}}=\hat{f}_{1}^{\prime}(0)$. Now

$$
\hat{f}_{1}^{\prime}(0)+r=\frac{(1-b)(1-r) e^{r \tau}}{(b-1) e^{r \tau}+(1-a) e^{\tau}}=\frac{(1-b)(1-r)}{(b-1)+(1-a) e^{\tau(1-r)}}>0 .
$$

Thus the hypothesis $-\frac{q_{1}}{p_{2}}>-r$ of Lemma 2 is satisfied. Now, let $\mathcal{L}$ be the line through $\left(0, q_{1}\right)$ and $\left(p_{2}, 0\right)$ and let $\mathcal{L}_{0}$ be the connected component of $\mathbb{R}^{2} \backslash \mathcal{L}$ that contains the origin. Lemma 2 guarantees $\hat{T}\left(\hat{\mathcal{D}}_{2}^{\prime}\right) \subset \mathcal{L}_{0}$. Also, note $\hat{T}\left(\hat{\mathcal{D}}_{2}^{\prime}\right)$ is linearly ordered in the $\preceq_{\text {se }}$ partial order, which we verify next. We may write $\hat{\mathcal{D}}_{2}^{\prime}=$ $\left\{\left(p_{2} t,(1-t) q_{1}\right): 0 \leq t \leq 1\right\}$. For $0 \leq t \leq 1$ set

$$
\begin{aligned}
(\tilde{u}(t), \tilde{v}(t)) & :=\hat{T}\left(\left(p_{2} t,(1-t) q_{1}\right)\right) \\
& =\left(\ln \left(a e^{p_{2} t}+(1-a) e^{p_{2} t+(1-t) q_{1}}\right), \ln \left(\frac{e^{(1-t) q_{1}}}{(1-b) e^{p_{2} t+(1-t) q_{1}}+b}\right)\right)
\end{aligned}
$$

then $\hat{T}\left(\hat{\mathcal{D}}_{2}^{\prime}\right)=\{(\tilde{u}(t), \tilde{v}(t)): 0 \leq t \leq 1\}$. From $(51)$,

$$
\frac{d \tilde{u}}{d t}=\frac{a p_{2}+(1-a)\left(p_{2}-q_{1}\right) e^{(1-t) q_{1}}}{a+(1-a) e^{(1-t) q_{1}}} \quad \text { and } \quad \frac{d \tilde{v}}{d t}=-\frac{p_{2}(1-b) e^{q_{1}+p_{2} t}+b q_{1} e^{q_{1} t}}{(1-b) e^{q_{1}+p_{2} t}+b e^{q_{1} t}} .
$$

Using statements (i) and (ii) of Claim 1 and (52) we conclude that for $\tau$ large enough, $\tilde{u}(t)$ is a decreasing function of $t \in[0,1]$ and $\tilde{v}(t)$ is an increasing function of $t \in[0,1]$. Consequently, $\hat{T}\left(\hat{\mathcal{D}}_{2}^{\prime}\right)$ is a curve linearly ordered in the $\preceq_{\text {se }}$ partial order and is thus a subset of the rectangular region $\mathcal{R}$ determined by the initial and final points. Hence

$$
\hat{T}\left(\hat{\mathcal{D}}_{2}^{\prime}\right) \subset \mathcal{R} \cap \mathcal{L}_{0}
$$

Note that $\hat{T}\left(0, q_{1}\right) \in \mathcal{K}_{\tau}$ by Claim 4 and $\hat{T}\left(p_{2}, 0\right) \in \mathcal{K}_{\tau}$ by Lemma 3 . It follows from (53) and the convexity of $\mathcal{K}_{\tau}$ that $\hat{T}\left(\hat{\mathcal{D}}_{2}^{\prime}\right) \subset \mathcal{K}_{\tau}$. 
Claim 6 For all $\tau$ large enough, $\hat{T}\left(\hat{\mathcal{D}}_{3}^{\prime}\right) \subset \mathcal{K}_{\tau}$.

Proof. Suppose $(u, v) \in \hat{\mathcal{D}}_{3}^{\prime}$. From $(23)$,

$$
\hat{T}(u, v)=\left(\ln \left(a e^{u}+(1-a) e^{u+v}\right), \ln \left(\frac{e^{v}}{(1-b) e^{u+v}+b}\right)\right) .
$$

By statement (41) in Lemma 3,

$$
\hat{T}(u, v) \in\left\{(s, t): s>c_{2} t^{2}+c_{1} t+c_{0}, t>0\right\} .
$$

Now, let $\mathcal{L}$ be the line through $\left(0, q_{2}\right)$ and $(\tau, 0)$. Then $\mathbb{R}^{2} \backslash \mathcal{L}$ has two connected components, one of which, $\mathcal{L}_{0}$, contains the origin. As a result of (54), to complete the proof it suffices to verify that $\hat{T}(u, v)$ belongs to $\mathcal{L}_{0}$. In other words, for $\tau$ large enough,

$$
\frac{1}{\tau} \ln \left(a e^{u}+(1-a) e^{u+v}\right)+\frac{1}{q_{2}} \ln \left(\frac{e^{v}}{(1-b) e^{u+v}+b}\right)<1 .
$$

Set

$$
\Delta_{\tau}:=1-\frac{1}{\tau} \ln \left(a e^{u}+(1-a) e^{u+v}\right)-\frac{1}{q_{2}} \ln \left(\frac{e^{v}}{(1-b) e^{u+v}+b}\right) .
$$

Then (55) is equivalent to

$$
\Delta_{\tau}>0
$$

for $\tau$ large enough. Consider the sets $\mathcal{Q}_{+}$and $\mathcal{Q}_{-}$defined in (45). We verify (57) for $(u, v) \in \mathcal{Q}_{-}$and for $(u, v) \in \mathcal{Q}_{+}$separately. Suppose $(u, v) \in \mathcal{Q}_{-}$. In this case, $a e^{u}+(1-a) e^{u+v}$ is a weighted average of two numbers that are less than 1 which implies that $\ln \left(a e^{u}+(1-a) e^{u+v}\right)<0$. Consequently, $\hat{T}(u, v) \in\{(s, t): s \leq 0, t>$ 0\}. Combining this with (54), (57) follows. Now suppose $(u, v) \in \mathcal{Q}_{+}$. From (56),

$$
\begin{aligned}
\tau q_{2} \Delta_{\tau} & =\tau q_{2}-q_{2} \ln \left(a e^{u}+(1-a) e^{u+v}\right)-\tau \ln \left(\frac{e^{v}}{(1-b) e^{u+v}+b}\right) \\
& =\tau\left(q_{2}-v\right)+\tau \ln \left((1-b) e^{u+v}+b\right)-q_{2}(u+v)-q_{2} \ln \left(a e^{-v}+1-a\right) .
\end{aligned}
$$


By convexity, $e^{(1-b)(u+v)+b \cdot 0} \leq(1-b) e^{u+v}+b e^{0}$. That is,

$$
(1-b)(u+v) \leq \ln \left((1-b) e^{u+v}+b\right)
$$

Recognizing that $\ln \left(a e^{-v}+1-a\right)<0$ for $v \geq 0$ and combining (58) and (59),

$$
\begin{aligned}
\tau q_{2} \Delta & \geq \tau\left(q_{2}-v\right)+\tau(1-b)(u+v)-q_{2}(u+v) \\
& =\tau\left(q_{2}-v\right)+(u+v)\left(\tau(1-b)-q_{2}\right) .
\end{aligned}
$$

From Claim 1, we can consider $\tau$ large enough such that $q_{2}<(1-b) \tau$. Therefore, since $u+v>0$ and $0<v \leq q_{2}$, (60) implies $\Delta_{\tau} \geq 0$. If $\Delta_{\tau}=0$, then (60) implies $v=q_{2}$ and $u+v=0$, which contradicts $(u, v) \in \mathcal{Q}_{+}$. Consequently, (57) holds.

Claim 7 For all $\tau$ large enough, $\hat{T}\left(\hat{\mathcal{D}}_{4}^{\prime}\right) \subset \mathcal{K}_{\tau}$.

Proof. We have $\hat{\mathcal{D}}_{4}^{\prime}=\left\{\left(t \tau,(1-t) q_{2}\right): t \in[0,1]\right\}$. For $t \in[0,1]$, let $(\tilde{u}(t), \tilde{v}(t))$ be given by

$$
\begin{aligned}
(\tilde{u}(t), \tilde{v}(t)) & =\hat{T}\left(t \tau,(1-t) q_{2}\right) \\
& =\left(\ln \left(a e^{t \tau}+(1-a) e^{t \tau+(1-t) q_{2}}\right), \ln \left(\frac{e^{(1-t) q_{2}}}{(1-b) e^{t \tau+(1-t) q_{2}}+b}\right)\right) .
\end{aligned}
$$

From (61),

$$
\begin{aligned}
& \frac{d \tilde{u}}{d t}=\frac{(1-a)\left(\tau-q_{2}\right) e^{q_{2}}+a \tau e^{q_{2} t}}{(1-a) e^{q_{2}}+a e^{q_{2} t}} \\
& \frac{d \tilde{v}}{d t}=-\frac{\tau e^{q_{2}+t \tau}(1-b)+b q_{2} e^{q_{2} t}}{b e^{q_{2} t}+(1-b) e^{q_{2}+t \tau}}
\end{aligned}
$$

Using statement (iii) of Claim 1 along with (62) and (63), we can conclude for $\tau$ large enough that $\tilde{u}(t)$ is an increasing function of $t \in[0,1]$ and $\tilde{v}(t)$ is a decreasing function of $t \in[0,1]$. As a consequence,

$$
\hat{T}\left(\hat{\mathcal{D}}_{4}^{\prime}\right) \text { is linearly ordered in the } \preceq_{\text {se }} \text { partial order. }
$$


We also have

$$
\begin{aligned}
\hat{T}(\tau, 0) & =\left(\ln \left(a e^{\tau}+(1-a) e^{\tau}\right), \ln \left(\frac{1}{(1-b) e^{\tau}+b}\right)\right) \\
& =\left(\tau,-\ln \left((1-b) e^{\tau}+b\right)\right) \in \hat{\mathcal{D}}_{0}^{\prime}
\end{aligned}
$$

In light of (64) and (65), to prove the claim, it is sufficient to verify that $\hat{T}\left(\hat{\mathcal{D}}_{4}^{\prime}\right)$ is in a suitable component of the complement of the line through $\left(0, q_{2}\right)$ and $(\tau, 0)$, for $\tau$ large enough. More precisely, we wish to verify

$$
\frac{1}{\tau} \ln \left(a e^{t \tau}+(1-a) e^{t \tau+(1-t) q_{2}}\right)+\frac{1}{q_{2}} \ln \left(\frac{e^{(1-t) q_{2}}}{(1-b) e^{t \tau+(1-t) q_{2}}+b}\right)<1 .
$$

For fixed $\tau$, define

$$
\psi_{\tau}(t):=q_{2} \ln \left(a+(1-a) e^{(1-t) q_{2}}\right)-\tau \ln \left((1-b) e^{t \tau+(1-t) q_{2}}+b\right) .
$$

Equation (66) is equivalent to

$$
\psi_{\tau}(t)<0 \text { for } \quad 0 \leq t \leq 1
$$

We have,

$$
\psi_{\tau}^{\prime}(t)=-e^{(1-t) q_{2}}\left(\frac{(1-b) \tau\left(\tau-q_{2}\right) e^{t \tau}}{b+(1-b) e^{t \tau+(1-t) q_{2}}}+\frac{(1-a) q_{2}^{2}}{a+(1-a) e^{(1-t) q_{2}}}\right)<0
$$

Finally, $\psi_{\tau}(0)<0$ follows from Claim 6 and thus (68) implies (67).

This completes the proof of Proposition 3 and thus, by Corollary 4, of statement (vii) from Theorem 1.

\section{List of References}

[1] L. J. S. Allen, An Introduction to Mathematical Biology. Upper Saddle River, New Jersey: Pearson Prentice Hall, 1987.

[2] M. Gidea, J. D. Meiss, I. Ugarcovici, and H. Weiss, "Applications of kam thoery to population dynamics," Journal of Biological Dynamics, vol. 1, pp. $1-23,2009$. 
[3] B. Hawkins, Pattern and Process in Host-Parasitoid Interactions. New York: Cambridge University Press, 1994.

[4] M. Hassell, The Spatial and Temporal Dynamics of Host-Parasitoid Interactions. New York: Oxford University Press, 2000.

[5] T. Wang and Y. Zhang, "Chemical control for host-parasitoid model within the parasitism season and its complex dynamics," Discrete Dynamics in $\mathrm{Na}$ ture and Society, vol. 1, pp. 1-14, 2016.

[6] C. Xiang and Z. Xiang, "Discete switching host-parasitoid models with integrated pest control," International Journal of Bifurcation and Chaos, vol. 24, pp. 1-20, 2014.

[7] Q. Din, "Global behavior of a host-parasitoid model under the constant refuge effect," Applied Mathematical Modeling, vol. 40, pp. 2815-2826, 2016.

[8] Y. Kang, S. Sasmal, A. Bhowmick, and J. Chattopadhyay, "A host-parasitoid system with predation-driven component allee effects in host population," Journal of Biological Dynamics, vol. 9, pp. 213-232, 2015.

[9] G. Livadiotis, L. Assas, B. Dennis, S. Elaydi, and E. Kwessi, "A discrete-time host-parasitoid model with an allee effect," Journal of Biological Dynamics, vol. 9, pp. 34-51, 2015.

[10] R. M. May, "Host-parasitoid systems in patchy environments: A phenomenological model," Journal of Animal Ecology, vol. 17, pp. 833-844, 1978.

[11] J. Yang, S. Tang, and Y. Tan, "Complex dynamics and bifurcation analysis of host-parasitoid models with impulsive control strategy," Chaos, Solitons and Fractals, vol. 91, pp. 522-532, 2016.

[12] Q. Din and T. Donchev, "Global character of a host-parasite model," Chaos, Solitons 85 Fractals, vol. 54, pp. 1-7, 2013.

[13] Y. Cai, Z. Wang, and W. Wang, "Endemic dynamics in a host-parasite epidemiological model within spatially heterogeneous environment," Applied Mathematics Letters, vol. 61, pp. 129-136, 2016.

[14] A. Gómez-Corral and M. L. García, "Control strategies for a stochastic model of host-parasite interaction in a seasonal environment," Journal of Theoretical Biology, vol. 354, pp. 1-11, 2014.

[15] C. McCaig, A. Fenton, A. Graham, C. Shankland, and R. Norman, "Using process algebra to develop predator-prey models of within-host parasite dynamics," Journal of Theoretical Biology, vol. 329, pp. 74-81, 2013. 
[16] Y. Tang, "Global dynamics of a parasite-host model with nonlinear incidence rate," International Journal of Bifurcation and Chaos, vol. 25, pp. 1-9, 2015.

[17] U. Ufuktepe and S. Kapçak, "Stability analysis of a host-parasite model," Advances in Difference Equations, vol. 79, pp. 1-9, 2013.

[18] P. H. Leslie, "Some further notes on the use of matrices in population mathematics," Biometrika, vol. 55, pp. 213-245, 1948.

[19] P. Leslie and J. Gower, "The properties of a stochastic model for two competing species," Biometrika, vol. 45, pp. 316-330, 1958.

[20] M. Nurkanović, "Asymptotic behavior of solutions to some two-dimensional systems of difference equations with applications," Ph.D. dissertation, University of Sarajevo, 2002.

[21] M. R. S. Kulenović and M. Nurkanović, "Asymptotic behavior of a twodimensional linear fractional system of difference equations," Radovi Mathematićki, vol. 11, pp. 1-19, 2002.

[22] M. R. S. Kulenović and O. Merino, "A global attractivity result for maps with invariant boxes," Discrete and Continuous Dynamical Systems, vol. 6, pp. 97-110, 2006.

[23] M. R. S. Kulenović and O. Merino, Discrete Dynamical Systems and Difference Equations with Mathematica. Boca Raton: Chapman\& Hall/CRC Press, 2002.

[24] U. Krause, Positive Dynamical Systems in Discrete Time. Berlin: De Gruyter, 2015.

[25] W. T. Jamieson and O. Merino. University of Rhode Island. "Local dynamics of planar maps with a non-isolated fixed point exhibiting 1-1 resonance." 2017. [Online]. Available: http://math.uri.edu/ merino/JamiesonMerino2017.pdf

[26] H. L. Smith, "Planar competitive and cooperative difference equations," Journal of Difference Equations and Applications, vol. 3, pp. 335-357, 1998. 


\title{
MANUSCRIPT 4 \\ Results on the Dynamics of a Class of Discrete Time Structured Population Models
}

\author{
David T. McArdle and Orlando Merino
}

Publication Status:

In preparation to be submitted to Advances in Difference Equations

Keywords: difference equation, global asymptotic stability, structured population model

AMS Subject Classification: Primary: 39A10, 39A11 


\section{Abstract}

Suppose $k$ is a given positive natural number, $M$ is a $k \times k$ matrix with nonnegative entries, $f_{\ell}:[0, \infty) \rightarrow[0, \infty)$ is a continuous function with $f_{\ell}(0)=0$ for $\ell \in\{1, \ldots, k\}, A$ is a bounded, linear operator on an ordered Banach space $X$ with positive cone $X_{+}$such that $A X_{+} \subset X_{+}$, and for each $\ell \in\{1, \ldots, k\}, \mathrm{b}_{\ell} \in X_{+}$ and $c_{\ell}$ is a positive bounded linear functional on $X$.

Consider the following systems of difference equations:

$$
\mathrm{y}_{n+1}=M\left(f_{1}\left(\mathrm{y}_{n}^{(1)}\right), \ldots, f_{k}\left(\mathrm{y}_{n}^{(k)}\right)\right)^{t}, \quad n=0,1,2, \ldots, \quad \mathrm{y}_{0} \in \mathbb{R}_{+}^{k}
$$

and

$$
\mathrm{x}_{n+1}=A \mathrm{x}_{n}+\sum_{\ell=1}^{k} f_{\ell}\left(\mathrm{c}_{\ell} \mathrm{x}_{n}\right) \mathrm{b}_{\ell}, \quad n=0,1,2, \ldots, \quad \mathrm{x}_{0} \in X_{+} .
$$

Conditions are established under which there is a correspondence between equilibrium points of (I) and (II). Under these conditions, and when $X=\mathbb{R}^{m}$, the stability type of the zero equilibrium of $(\mathrm{I})$ is shown to be the same as that for (II). When $k=2$ and the functions $f_{\ell}$ have certain monotonicity and convexity characteristics, sufficient conditions are given for the existence of a unique positive equilibrium for system (I). In this case, the stability of the equilibrium at the origin is also established. Examples are included.

\subsection{Introduction}

Discrete dynamical systems are used to model populations in biological, epidemiological, and entomological applications. Many of these populations are nonhomogeneous in the sense that individuals vary in physiological characteristics and may interact with the environment differently. These differences play an important role in the dynamics of the entire population and necessitate a special type of mathematical model, referred to as a structured population model. Structured population models divide a population into specific classes or categories based 
on, among other things, chronological age, body size, or genetic differences [1]. The model then tracks the size/density through each generation for the variety of classes by utilizing information related to the growth within each class as well as rates of transfer from one class to another [1,2]. Models of this type have also been referred to as compartmental models and are discussed in detail in [1] and [2]. For interesting applications of structured models that have been studied recently, see [3], [4], [5], and [6]. The focus of this manuscript will be on two specific types of structured population models, introduced next.

Suppose that a population is divided into $k$ classes so that at (discrete) time $n \in \mathbb{N}$ the (density, number of individuals, etc.) in class $\ell$ is $\mathrm{y}_{n}^{(\ell)}$. Denote by $\mathrm{y}_{n}$ the vector in $\mathbb{R}_{+}^{k}$ such that

$$
\mathrm{y}_{n}=\left(\mathrm{y}_{n}^{(1)}, \mathrm{y}_{n}^{(2)}, \ldots, \mathrm{y}_{n}^{(k)}\right) .
$$

An increase of 1 unit over $n$ gives a new number of individuals in class $\ell$, which is determined based on the previous number of individuals in each of the $k$ classes. Assume that in the $(n+1)^{\text {st }}$ generation the population in class $\ell$ gets a contribution from each of the $k$ classes according to the formula

$$
\mathrm{y}_{n+1}^{(\ell)}=m_{\ell, 1} f_{1}\left(\mathrm{y}_{n}^{(1)}\right)+m_{\ell, 2} f_{2}\left(\mathrm{y}_{n}^{(2)}\right)+\cdots+m_{\ell, k} f_{k}\left(\mathrm{y}_{n}^{(k)}\right),
$$

where $f_{\ell}:[0, \infty) \rightarrow[0, \infty)$ is a continuous function with $f_{\ell}(0)=0$ for $\ell \in$ $\{1,2, \ldots, k\}$. The terms $m_{\ell, j}$ are nonnegative constants that reflect birth and death rates of the $\ell$ class and take into account transfer rates from the $j$ to $\ell$ class. If we denote by $M=\left(m_{\ell, j}\right)$ the $k \times k$ coefficient matrix, then we may write (1) as the system

$$
\mathrm{y}_{n+1}=M\left(f_{1}\left(\mathrm{y}_{n}^{(1)}\right), \ldots, f_{k}\left(\mathrm{y}_{n}^{(k)}\right)\right)^{t}, \quad n=0,1,2, \ldots, \quad \mathrm{y}_{0} \in \mathbb{R}_{+}^{k} .
$$

Systems of the form (I) were introduced for a specific genetics example by Friedland and Karlin in [7] and can also be thought of as a special case of the more general 
equation

$$
\mathrm{y}_{n+1}=P\left(\mathrm{y}_{n}\right) \mathrm{y}_{n}, \quad n=0,1,2, \ldots, \quad \mathrm{y}_{0} \in \mathbb{R}_{+}^{k},
$$

which is studied by J. M. Cushing in [1]. Model (I) is a primary focus of this manuscript.

Model (I) is useful in analyzing other types of structured population models. In particular, (I) is utilized in this paper to study systems of the form

$$
\mathrm{x}_{n+1}=A \mathrm{x}_{n}+\sum_{\ell=1}^{k} f_{\ell}\left(c_{\ell} \mathrm{x}_{n}\right) \mathrm{b}_{\ell}, \quad n=0,1,2, \ldots, \quad \mathrm{x}_{0} \in X_{+},
$$

where $A$ is a bounded, linear operator on an ordered Banach space $X$ with positive cone $X_{+}$such that $A X_{+} \subset X_{+}$and for each $\ell \in\{1, \ldots, k\}, \mathrm{b}_{\ell} \in X_{+}, \mathrm{c}_{\ell}$ is a positive bounded linear functional on $X$, and $f_{\ell}$ is a real valued function, as defined above.

Model (II) is a generalization of the population model formulated by Rebarber, Tenhumberg, and Townley in [8]. They introduce the nonlinear, density dependent model given by

$$
\mathrm{x}_{n+1}=A \mathrm{x}_{n}+f\left(\mathrm{cx}_{n}\right) \mathrm{b}, \quad n=0,1,2, \ldots, \quad \mathrm{x}_{0} \in X_{+},
$$

where $A, \mathrm{x}_{n}, \mathrm{c}, \mathrm{b}$ and $f$ are as stated above when $k=1$ (see [8]). System (2) successfully takes into account two important biological processes, survival/growth and fecundity, and is useful in modeling stage structured plant and fishery populations $[8,9]$. The operator $A$ is referred to as the survival operator and $f(\cdot) \mathrm{b}$ is referred to as the fecundity operator. The term $\mathrm{cx}_{n}$ describes the number (or density) of offspring, $f\left(\mathrm{cx}_{n}\right)$ reflects the nonlinear density dependence on $\mathrm{c}_{n}$, and b describes how the offspring are distributed amongst the classes. The density dependent fecundity structure $f\left(\mathrm{cx}_{n}\right)$ b is common for single-species, structured populations. Specific examples can be seen in [9], [10], and [11]. Some common 
density dependencies that are used in applications include

$$
\begin{array}{ll}
f(t)=\beta t^{\alpha}, & \alpha \in(0,1) \text { and } \beta>0, \\
f(t)=\frac{\gamma t}{\delta+t}, & \gamma, \delta>0, \\
f(t)=t e^{-\eta t}, & \eta>0,
\end{array}
$$

where (3) is a power-law nonlinearity, (4) is of Beverton-Holt type, and (5) is a Ricker nonlinearity [9]. Smith and Thieme in [12] also study (2) with general functions that satisfy monotonicity/concavity restrictions and are able to prove global dynamic results (see Theorems 6.1 and 7.1 in [12]). The current paper performs a similar analysis for (II). With system (II), applications can be studied involving structured populations that have $k$ subclasses within each class. For $\ell \in$ $\{1,2, \ldots, k\}$ the functions $f_{\ell}$ represent the nonlinear dependence on the offspring for each of these subclasses.

Model (II) is potentially set in a high dimensionality state space $X$. The nonlinearities, however, are of a very specific type. A natural question is whether it is possible to reduce the complexity of the problem to one where the dimension of the state space is the same as the number $k$ of nonlinearities that appear in (II). In this paper, we give an answer to the question by finding a $k$-dimensional model of the form (I) that can be used to study (II). In particular, in Section 4.5, we give conditions under which there is a one-to-one correspondence between the positive equilibrium points of (I) and (II). Positive equilibrium points represent persistence of the population and are an important feature of the models. Furthermore, when the state space of (II) is $\mathbb{R}^{m}$, we establish that the stability character of the origin (which represents the extinction state) in (I) is the same as that of (II). With these similarities between (I) and (II), it is useful to explore how stability of the zero equilibrium is related to the existence, uniqueness, and stability of a positive equilibrium for system (I). These results can then be used to draw conclusions 
about (II), hence reducing the complexity of the problem. For this reason, in Sections 4.3 and 4.4, we study dynamics of (I) and establish results related to the stability and existence and uniqueness of positive equilibria. Particular attention is given to the two-dimensional case when $k=2$.

The current paper is organized as follows: basic concepts and definitions are presented in Section 4.2 and general results regarding systems of the form (I) are presented in Section 4.3. The two-dimensional case of system (I) is analyzed in Section 4.4. In the two-dimensional case, when it is known that the functions $f_{1}, f_{2}$ satisfy certain monotonicity and convexity characteristics, sufficient conditions are established for the existence of a unique positive equilibrium. In this case, stability characteristics of the origin and the positive fixed point are established and examples are presented to illustrate the results. Systems of the form (II) are analyzed in Section 4.5 and connections are made with system (I) regarding the existence and uniqueness of positive equilibrium points and the stability of the origin.

\subsection{Background}

For convenience, basic notions and definitions are provided here that are utilized within the main sections of the paper.

Let $X$ be a Banach space over $\mathbb{R}$. A set $X_{+} \subset X$ is an order cone if $X_{+}$is closed, convex, and such that

$$
X_{+} \cap\left(-X_{+}\right)=\{0\}, X_{+} \neq\{0\}, \text { and } \lambda X_{+} \subset X_{+} \text {for all } \lambda \geq 0 \text {. }
$$

An order cone is solid if $\operatorname{int}\left(X_{+}\right) \neq \emptyset$ (see [13]). If $X^{*}$ is the dual space of $X$, the dual cone of $X_{+}$is the set

$$
X_{+}^{*}:=\left\{c \in X^{*}: c(\mathrm{x}) \geq 0, \text { for all } \mathrm{x} \in X_{+}\right\} .
$$

If $X_{+}$is solid, then the set $X_{+}^{*}$ is a cone in $X^{*}$. A functional $c \in X_{+}^{*}$ is said to be positive. The functional is strictly positive if $c(x)>0$ for $\mathrm{x} \in X_{+} \backslash\{0\}$. If 
$\mathrm{x} \in \operatorname{int}\left(X_{+}\right)$, then $c(\mathrm{x})>0$ for every $c \in X_{+}^{*} \backslash\{0\}$ ([13], Proposition 19.3).

Every order cone $X_{+}$induces a partial order $\preceq$ on $X$ as follows: $\mathrm{x} \preceq \mathrm{y}$ if and only if $\mathrm{y}-\mathrm{x} \in X_{+}$. In this case, $X$ is an ordered Banach space with order cone $X_{+}$. For points $\mathrm{x}, \mathrm{y} \in X_{+}$, we say $\mathrm{x} \preceq \mathrm{y}$ if and only if $\mathrm{y}-\mathrm{x} \in X_{+}, \mathrm{x} \prec \mathrm{y}$ if and only if $\mathrm{y}-\mathrm{x} \in X_{+} \backslash\{0\}$, and $\mathrm{x} \ll \mathrm{y}$ if and only if $\mathrm{y}-\mathrm{x} \in \operatorname{int}\left(X_{+}\right)$(see [14]). The partial order $\preceq$ is compatible with addition, multiplication by a nonnegative

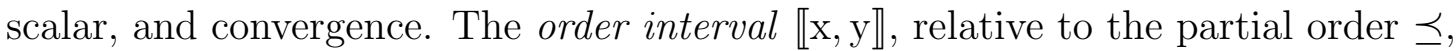
is defined by

$$
\llbracket \mathrm{x}, \mathrm{y} \rrbracket:=\{\mathrm{u} \in X: \mathrm{x} \preceq \mathrm{u} \preceq \mathrm{y}\}
$$

Let $T: X \rightarrow X$ be an operator. We say that $T$ is monotone (with respect to the partial order $\preceq$ ) if $\mathrm{x} \preceq \mathrm{y} \Longrightarrow T(\mathrm{x}) \preceq T(\mathrm{y})$, strictly monotone if $\mathrm{x} \prec \mathrm{y} \Longrightarrow$ $T(\mathrm{x}) \prec T(\mathrm{y})$ and strongly monotone if $\mathrm{x} \prec \mathrm{y} \Longrightarrow T(\mathrm{x}) \ll T(\mathrm{y})$. If $T$ is linear, monotonicity is equivalent to $T X_{+} \subset X_{+}$and strong monotonicity is equivalent to $T\left(X_{+} \backslash\{0\}\right) \subset \operatorname{int}\left(X_{+}\right)$. If an operator $T$ is monotone with respect to a partial order, it is referred to as order-preserving [14].

Let $\mathbb{R}^{m}=\left\{\left(x_{1}, \ldots, x_{m}\right): x_{\ell} \in \mathbb{R}, \ell=1, \ldots, m\right\}$. The following result from [14] is stated for order-preserving operators when $X$ is taken to be $\mathbb{R}^{m}$.

Theorem 1 For a nonempty set $U \in \mathbb{R}^{m}$ and a partial order $\preceq$ on $\mathbb{R}^{m}$, Let $T: U \rightarrow U$ be an order-preserving map and let $\mathrm{u}, \mathrm{v} \in U$ be such that $\mathrm{u} \prec \mathrm{v}$ and $\llbracket \mathrm{u}, \mathrm{v} \rrbracket \subset U$. If $\mathrm{u} \preceq T(\mathrm{u})$ and $T(\mathrm{v}) \preceq \mathrm{v}$, then $\llbracket \mathrm{u}, \mathrm{v} \rrbracket$ is an invariant set and

(i) There exists a fixed point of $T$ in $\llbracket \mathrm{u}, \mathrm{v} \rrbracket$.

(ii) If $T$ is strongly order-preserving, then there exists a fixed point of $T$ in $\llbracket \mathrm{u}, \mathrm{v} \rrbracket$ that is stable relative to $\llbracket \mathrm{u}, \mathrm{v} \rrbracket$.

(iii) If there is only one fixed point in $\llbracket \mathrm{u}, \mathrm{v} \rrbracket$, then it is a global attractor in $\llbracket \mathrm{u}, \mathrm{v} \rrbracket$ and therefore asymptotically stable relative to $\llbracket \mathrm{u}, \mathrm{v} \rrbracket$. 
Properties of matrices with real entries also play a role in the coming sections. A matrix $A \in \mathbb{R}^{m \times m}$ is nonnegative (positive), written $A \geq 0(A>0)$ if all of the entries of $A$ are nonnegative (positive). If $A>0$, then $A$ is strongly monotone as a linear operator on $\mathbb{R}^{m}$ with order cone $\mathbb{R}_{+}^{m}$. A nonnegative matrix $A$ is called primitive if there exists $N \in \mathbb{N}$ such that $A^{N}>0$. The matrix $A$ is irreducible if for any $i, j$ there is a $k=k(i, j)$ such that $\left(A^{k}\right)_{i j}>0$. If $A$ is irreducible, then $I+A$ is primitive (which can be shown by choosing $N$ sufficiently large and expanding $(I+A)^{N}$ in powers of $\left.A\right)$. Furthermore. if $A$ is irreducible and $\rho(A)<1$, then $(I-A)^{-1}>0\left(\right.$ which follows from $\left.(I-A)^{-1}=I+A+A^{2}+\cdots\right)[15]$.

Other useful results involve properties associated with the determinant of a matrix. If $A(t)$ is an $m \times m$ matrix valued, differentiable function of a real variable $t$, Jacobi's formula gives the derivative of the determinant of $A(t)$ in terms of the adjugate of $A(t)$ and the derivative of $A(t)$ :

$$
\frac{d}{d t} \operatorname{det} A(t)=\operatorname{tr}\left(\operatorname{adj}(A(t)) \frac{d A(t)}{d t}\right) .
$$

The adjugate of $A \in \mathbb{R}^{m \times m}$ satisfies $\operatorname{adj}(A) A=\operatorname{det}(A) I_{m}$, where $I_{m}$ is the $m \times m$ identity matrix. If $A$ is $m \times p$ and $B$ is an $p \times m$ matrix, Sylvester's determinant identity [16] is

$$
\operatorname{det}\left(I_{m}+A B\right)=\operatorname{det}\left(I_{p}+B A\right)
$$

\subsection{General Results for System (I)}

In this section we investigate systems of the form (I) on $\mathbb{R}_{+}^{k}$ with regards to stability of the zero equilibrium and existence, uniqueness, and stability of a positive equilibrium. Let $\preceq$ be a partial order on $\mathbb{R}^{k}$, where the order cone is taken to be the standard positive orthant $\mathbb{R}_{+}^{k}$. In other words, for $\mathrm{u}, \mathrm{v} \in \mathbb{R}^{k}, u \preceq v$ if and only if $\mathrm{u}^{(\ell)} \leq \mathrm{v}^{(\ell)}$ for all $\ell \in\{1,2, \ldots, k\}$. Let $T: \mathbb{R}_{+}^{k} \rightarrow \mathbb{R}_{+}^{k}$ be the map associated 
with system (I). That is, for $\mathrm{y} \in \mathbb{R}_{+}^{k}$,

$$
T(\mathrm{y})=M\left(f_{1}\left(\mathrm{y}^{(1)}\right), f_{2}\left(\mathrm{y}^{(2)}\right), \ldots, f_{k}\left(\mathrm{y}^{(k)}\right)\right)^{t},
$$

where $M \in \mathbb{R}_{+}^{k \times k}$ and $f_{\ell}:[0, \infty) \rightarrow[0, \infty)$ is a continuous function with $f_{\ell}(0)=0$ for $\ell \in\{1,2, \ldots, k\}$. For the remainder of the paper, we additionally assume that $f_{\ell}$ has a $C^{(1)}$ extension on a neighborhood of $[0, \infty)$ for $\ell \in\{1,2, \ldots, k\}$.

The map $T$ from (6) may be written in the form

$$
T(\mathrm{y})=M D(\mathrm{y}) \mathrm{y}
$$

where $D \in \mathbb{R}_{+}^{k \times k}$ is defined by

$$
D(\mathrm{y})=\operatorname{diag}\left(g_{1}\left(\mathrm{y}^{(1)}\right), \ldots, g_{k}\left(\mathrm{y}^{(k)}\right)\right)
$$

for

$$
g_{\ell}(t)=\left\{\begin{array}{cc}
\frac{f_{\ell}(t)}{t}, & \text { if } t>0 \\
f_{\ell}^{\prime}(0), & \text { if } t=0
\end{array}, \quad \ell \in\{1,2, \ldots, k\} .\right.
$$

It follows from (7) that $0 \in \mathbb{R}_{+}^{k}$ is a fixed point of $T$. The Jacobian matrix associated with $T$ is given by

$$
J_{T}(\mathrm{y})=M\left(f_{1}^{\prime}\left(\mathrm{y}^{(1)}\right), f_{2}^{\prime}\left(\mathrm{y}^{(2)}\right), \ldots, f_{k}^{\prime}\left(\mathrm{y}^{(k)}\right)\right)^{t} .
$$

Since $M$ consists of nonnegative entries, the following remark about the monotonicity of $T$ follows from (8).

Remark $1 T$ is monotone on $\mathbb{R}_{+}^{k}$ provided that $f_{\ell}$ is increasing for $\ell \in$ $\{1,2, \ldots, k\}$.

Global results for system (I) can be established when more information is known about the functions $f_{\ell}$ for $\ell \in\{1,2, \ldots, k\}$. Define,

$$
\begin{aligned}
\overline{f^{\prime}(\infty)} & =\max _{\ell}\left\{\limsup _{t \rightarrow \infty} \frac{f_{\ell}(t)}{t}\right\}, & \overline{f^{\prime}(0)} & =\max _{\ell}\left\{f_{\ell}^{\prime}(0)\right\} \\
\underline{f^{\prime}(\infty)} & =\min _{\ell}\left\{\liminf _{t \rightarrow \infty} \frac{f_{\ell}(t)}{t}\right\}, & \underline{f^{\prime}(0)} & =\min _{\ell}\left\{f_{\ell}^{\prime}(0)\right\} .
\end{aligned}
$$


In addition, denote by $h_{+}(M)$ the maximum absolute row sum of $M$ and by $h_{-}(M)$ the minimum absolute row sum of $M$. That is,

$$
h_{+}(M)=\max _{i} \sum_{j=1}^{k}\left|m_{i, j}\right| \quad \text { and } \quad h_{-}(M)=\min _{i} \sum_{j=1}^{k}\left|m_{i, j}\right| .
$$

For notational simplicity, let

$$
\mathrm{e}:=(1,1, \ldots, 1)^{t} \in \mathbb{R}_{+}^{k}
$$

Lemmas 1 and 2, presented next, help to establish results related to the existence of a positive fixed point for the map $T$ as well as to the stability of the origin. Lemma 1 provides sufficient conditions for the existence of a strict supersolution (i.e. $\mathrm{y} \in \operatorname{int}\left(\mathbb{R}_{+}^{k}\right)$ such that $T(\mathrm{y}) \prec \mathrm{y}$ ) or a strict subsolution (i.e. $\mathrm{y} \in \operatorname{int}\left(\mathbb{R}_{+}^{k}\right)$ such that $\mathrm{y} \prec T(\mathrm{y}))$ where $\|\mathrm{y}\|>r$ for $r$ arbitrarily large. These solutions provide valuable information about the behavior of the map $T$ at infinity. Lemma 2 presents a similar result with y as close to the origin as we wish.

Lemma 1 Let $r>0$. The following properties hold:

(i) If $0 \leq \overline{f^{\prime}(\infty)} h_{+}(M)<1$, then there exists $t^{*}>r$ such that for all $t>t^{*}$, $\mathrm{y}=t$ e satisfies $T(\mathrm{y}) \prec \mathrm{y}$.

(ii) If $\underline{f^{\prime}(\infty)} h_{-}(M)>1$, then there exists $t^{*}>r$ such that for all $t>t^{*}, \mathrm{y}=t \mathrm{e}$ satisfies $\mathrm{y} \prec T(\mathrm{y})$.

Proof.

(i) Let $r>0$ and suppose that the functions $f_{\ell}$ satisfy $0 \leq \overline{f^{\prime}(\infty)} h_{+}(M)<1$. Choose $t^{*}$ such that

$$
t^{*}>r \quad \text { and } \max _{\ell}\left\{\frac{f_{\ell}(t)}{t}\right\}<\frac{1}{h_{+}(M)} \quad \text { for all } t>t^{*} .
$$

Therefore, for all $t>t^{*}$,

$$
D(t \mathrm{e}) \mathrm{e}=\operatorname{diag}\left(\frac{f_{1}(t)}{t}, \ldots, \frac{f_{k}(t)}{t}\right) \mathrm{e} \prec \frac{1}{h_{+}(M)} \mathrm{e} .
$$


Also, by the definition of $h_{+}(M), \frac{M}{h_{+}(M)} \mathrm{e} \prec$ e. Consequently, defining $\mathrm{y}=t \mathrm{e}$ and using the form of $T$ given in (7), we have

$$
T(\mathrm{y})=M D(\mathrm{y}) \mathrm{y}=M D(t \mathrm{e}) t \mathrm{e} \prec \frac{M}{h_{+}(M)} t \mathrm{e} \prec t \mathrm{e}=\mathrm{y} .
$$

(ii) Similarly, suppose that the functions $f_{\ell}$ satisfy $\underline{f^{\prime}(\infty)} h_{-}(M)>1$. Choose $t^{*}$ such that

$$
t^{*}>r \quad \text { and } \min \left\{f_{\ell}(t)\right\}>\frac{1}{h_{-}(M)} \text { for all } t>t^{*}
$$

Therefore, for all $t>t^{*}$,

$$
D(t \mathrm{e}) \mathrm{e}=\operatorname{diag}\left(\frac{f_{1}(t)}{t}, \ldots, \frac{f_{k}(t)}{t}\right) \text { e } \succ \frac{1}{h_{-}(M)} \mathrm{e} .
$$

Also, by the definition of $h_{-}(M), \frac{M}{h_{-}(M)}$ e $\succ$ e. Consequently, defining $\mathrm{y}=t \mathrm{e}$ and using the form of $T$ given in (7), we have

$$
T(\mathrm{y})=M D(\mathrm{y}) \mathrm{y}=M D(t \mathrm{e}) t \mathrm{e} \succ \frac{M}{h_{-}(M)} t \mathrm{e} \succ t \mathrm{e}=\mathrm{y}
$$

The result follows.

Lemma 2 Let $\delta>0$. The following properties hold:

(i) If $0 \leq \overline{f^{\prime}(0)} h_{+}(M)<1$, then there exists $t^{*}<\delta$ such that for all $t<t^{*}$, $\mathrm{y}=t$ e satisfies $T(\mathrm{y}) \prec \mathrm{y}$.

(ii) If $\underline{f^{\prime}(0)} h_{-}(M)>1$, then there exists $t^{*}<\delta$ such that for all $t<t^{*}, \mathrm{y}=t \mathrm{e}$ satisfies $\mathrm{y} \prec T(\mathrm{y})$.

Proof. The proofs of (i) and (ii) in Lemma 2 are similar to the proofs of (i) and (ii) in Lemma 1 and are omitted here.

The existence of sub and supersolutions allow for monotonicity properties of the map $T$ to be exploited, which leads to two theorems. Theorem 2 treats the local stability character of the fixed point at the origin and Theorem 3 is helpful in determining when a positive fixed point of the map $T$ is a global attractor.. 
Theorem 2 The following statements are true:

(i) If $0 \leq \overline{f^{\prime}(0)} h_{+}(M)<1$, then 0 is stable.

(ii) If $f^{\prime}(0) h_{-}(M)>1$, then 0 is unstable.

Proof. Statement (i) and (ii) follow directly from Lemma 2 and the definition of stable and unstable.

Theorem 3 Suppose $M$ is irreducible and, for $\ell \in\{1,2, \ldots, k\}, f_{\ell}$ is nondecreasing and such that $f_{\ell}^{\prime}(0)>0$. If $\rho\left(J_{T}(0)\right)>1$ and $0 \leq \overline{f^{\prime}(\infty)} h_{+}(M)<1$, then there exists at least one positive fixed point $\overline{\mathrm{x}}$ of the map T. Furthermore, if $\overline{\mathrm{x}}$ is unique then it is a global attractor on $\operatorname{int}\left(\mathbb{R}_{+}^{k}\right)$.

Proof. We claim that for all $\epsilon>0$, there exists $\mathrm{y}_{\epsilon} \in \operatorname{int}\left(\mathbb{R}_{+}^{k}\right)$ such that $\left\|\mathrm{y}_{\epsilon}\right\|<\epsilon$ and $\mathrm{y}_{\epsilon} \prec T\left(\mathrm{y}_{\epsilon}\right)$. Let $\epsilon>0$. Since $f_{\ell} \in C^{(1)}$ for $\ell \in\{1,2, \ldots, k\}$, the Taylor expansion of $T$ about 0 is

$$
T(\mathrm{y})=T(0)+J_{T}(0) \mathrm{y}+o(|\mathrm{y}|) .
$$

The irreducibility of $M$, along with $f_{\ell}^{\prime}(0)>0$ for $\ell \in\{1,2, \ldots, k\}$, implies that $J_{T}(0)$ is irreducible. Since $\rho\left(J_{T}(0)\right)>1$, by the Perron-Frobenius theorem [15], $\rho^{*}=\rho\left(J_{T}(0)\right)$ is an eigenvalue of $J_{T}(0)$ with an associated positive eigenvector $\mathrm{v}$. That is,

$$
J_{T}(0) \mathrm{v}=\rho^{*} \mathrm{v} .
$$

For $\alpha>0$, take $\mathrm{y}=\alpha \mathrm{v}$ in (9). Consequently,

$$
\begin{aligned}
T(\alpha \mathrm{v}) & =J_{T}(0) \alpha \mathrm{v}+o(|\alpha \mathrm{v}|) \\
& =\rho^{*} \alpha \mathrm{v}+o(|\alpha|)
\end{aligned}
$$


and therefore,

$$
\frac{1}{\alpha}(T(\alpha \mathrm{v})-\alpha \mathrm{v})=\left(\rho^{*}-1\right) \mathrm{v}+o(1)
$$

The relation in (11) implies that if $\alpha$ is chosen small enough, then $\alpha \mathrm{v} \prec T(\alpha \mathrm{v})$. This $\alpha$ can also be chosen in such a way that $\|\alpha \mathrm{v}\|<\epsilon$. Let $\mathrm{y}_{\epsilon}=\alpha \mathrm{v}$ and this proves the claim.

Now, since $0 \leq \overline{f^{\prime}(\infty)} h_{+}(M)<1$, then by Lemma 1 , there exists $\mathrm{y} \in \mathbb{R}_{+}^{k}$

such that $T(\mathrm{y}) \prec \mathrm{y}$. It follows from Theorem 1 that the order interval $\llbracket \mathrm{y}_{\epsilon}, \mathrm{y} \rrbracket$ is invariant under the map $T$ and there must exist at least one positive fixed point $\overline{\mathrm{x}}$ in $\llbracket \mathrm{y}_{\epsilon}, \mathrm{y} \rrbracket$.

Suppose that the positive fixed point $\overline{\mathrm{x}}$ of the map $T$ is unique. By statement (iii) of Theorem $1, \overline{\mathrm{x}}$ is a global attractor in the order interval « $\mathrm{y}_{\epsilon}, \mathrm{y} \rrbracket$. By Lemma 1, y can be chosen such that $\|\mathrm{y}\|>r$ for any $r>0$ and by the above claim, $\mathrm{y}_{\epsilon}$ can be chosen such that $\left\|\mathrm{y}_{\epsilon}\right\|<\epsilon$ for any $\epsilon>0$. Letting $\epsilon \rightarrow 0$ and $r \rightarrow \infty$, it follows that $\overline{\mathrm{x}}$ is a global attractor on $\operatorname{int}\left(\mathbb{R}_{+}^{k}\right)$.

We now explore the special case when $k=2$ for system (I).

\subsection{The Two-Dimensional Case for System (I)}

In the two-dimensional case, more information can be determined about the fixed points of the map $T$ in (6). Denote by $\tilde{T}$ the map in (6) when $k=2$. That is, with $M=\left(m_{i, j}\right) \in \mathbb{R}_{+}^{2 \times 2}$,

$$
\tilde{T}\left(\begin{array}{l}
u \\
v
\end{array}\right)=M\left(f_{1}(u), f_{2}(v)\right)^{t}=\left(\begin{array}{l}
m_{11} f_{1}(u)+m_{12} f_{2}(v) \\
m_{21} f_{1}(u)+m_{22} f_{2}(v)
\end{array}\right) .
$$

In the proceeding arguments, when it is assumed that $f_{1}, f_{2}:[0, \infty) \rightarrow[0, \infty)$ are twice differentiable, strictly increasing, and convex (concave), what is meant is that $f_{1}, f_{2}$ have extensions to functions with these properties that are defined on a neighborhood of $[0, \infty)$. Note that with this understanding, if $f_{1}, f_{2}$ are strictly 
increasing and concave (or convex), then

$$
f_{1}^{\prime}(t)>0 \text { and } f_{2}^{\prime}(t)>0 \text { for } t \geq 0 .
$$

In this section we determine conditions for the existence of a unique positive equilibrium and for the stability, or lack thereof, of the origin.

A natural problem is the following: Find conditions on $f_{1}, f_{2}$ under which there is a correspondence between the existence of a unique positive fixed point and the stability character of the origin. In Section 4.4.3, we give a complete answer for the specific case when $f_{1}, f_{2}$ are of Beverton-Holt type. For functions $f_{1}$ and $f_{2}$ satisfying more general conditions, the question is partly answered by Theorems 6 and 7 in Section 4.4.2, but the complete answer remains open. The question posed above in a higher dimensional setting is also of great interest.

\subsubsection{Conditions for Uniqueness of the Positive Equilibrium}

In this section we give conditions for which the map $\tilde{T}$ in (12) has a unique positive fixed point under restrictions placed on $f_{1}, f_{2}$. The equilibrium curves of the map $\tilde{T}$ are the curves denoted by $\mathcal{C}_{1}$ and $\mathcal{C}_{2}$, where

$$
\mathcal{C}_{\ell}: \quad t_{\ell}=\phi_{\ell}\left(t_{1}, t_{2}\right)=m_{\ell, 1} f_{1}\left(t_{1}\right)+m_{\ell, 2} f_{2}\left(t_{2}\right), \quad \ell=1,2 .
$$

Fixed points of the map $\tilde{T}$ occur at the intersection points of the equilibrium curves $\mathcal{C}_{1}$ and $\mathcal{C}_{2}$. We focus on two special cases:

Case 1: $f_{1}, f_{2}$ are twice differentiable, strictly increasing, and convex.

Case 2: $f_{1}, f_{2}$ are twice differentiable, strictly increasing, and concave.

Two theorems are now presented, which provide necessary and sufficient con-

ditions for a unique positive fixed point of $\tilde{T}$ to exist in Cases 1 and 2 from (14). We begin with Theorem 4, which involves Case 1. 
Theorem 4 Let $M>0$ and suppose that $f_{1}, f_{2}$ are twice differentiable, strictly increasing, and convex. Consider the following conditions:

(A) $\max \left\{m_{11} f_{1}^{\prime}(0), m_{22} f_{2}^{\prime}(0)\right\}<1$ and $\frac{1-m_{22} f_{2}^{\prime}(0)}{m_{12} f_{2}^{\prime}(0)}>\frac{m_{21} f_{1}^{\prime}(0)}{1-m_{11} f_{1}^{\prime}(0)}$.

(B1) There exists $t^{*}>0$ such that $m_{11} f_{1}^{\prime}\left(t^{*}\right)=1$.

(B2) There exists $t^{*}>0$ such that $m_{22} f_{2}^{\prime}\left(t^{*}\right)=1$.

(B3) The limits $L_{1}$ and $L_{2}$ exist and $L_{2}<L_{1}$, where

$$
L_{1}:=\lim _{t \rightarrow \infty}\left[\frac{m_{21} f_{1}^{\prime}(t)}{1-m_{11} f_{1}^{\prime}(t)}\right] \text { and } L_{2}:=\lim _{t \rightarrow \infty}\left[\frac{1-m_{22} f_{2}^{\prime}(t)}{m_{12} f_{2}^{\prime}(t)}\right] .
$$

The map $\tilde{T}$ has a positive fixed point $\left(t_{1}, t_{2}\right)$ if and only if $(\mathrm{A})$ and at least one of (B1), (B2), or (B3) are satisfied. When a positive fixed point $\left(t_{1}, t_{2}\right)$ exists, it is unique. Furthermore, in this case, $\left(t_{1}, t_{2}\right)$ and the origin are both hyperbolic fixed points.

Remark 2 It follows from (13) that the limit $L_{2}$ exists and the inequality in (A) is well defined. Also, if conditions (B1) and (B2) are not satisfied, then the monotonicity and convexity characteristics of $f_{1}, f_{2}$, along with $(\mathrm{A})$, imply that

$$
\lim _{t \rightarrow \infty}\left(m_{11} f_{1}^{\prime}(t)\right)<1 \quad \text { and } \quad \lim _{t \rightarrow \infty}\left(m_{22} f_{2}^{\prime}(t)\right)<1
$$

in which case the limit $L_{1}$ in (B3) exists.

Proof. Consider the parametric curves

$$
\phi_{\ell}:=\left\{\left(x_{\ell}(t), y_{\ell}(t)\right): t>0\right\}, \quad \ell=1,2,
$$

where

$$
\begin{aligned}
& x_{1}(t)=t-m_{11} f_{1}(t), \quad x_{2}(t)=m_{12} f_{2}(t), \\
& y_{1}(t)=m_{21} f_{1}(t), \quad y_{2}(t)=t-m_{22} f_{2}(t) \text {. }
\end{aligned}
$$




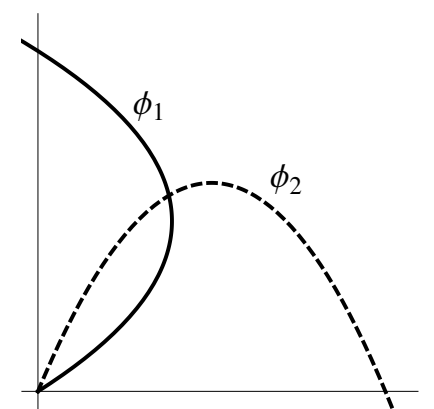

(a)

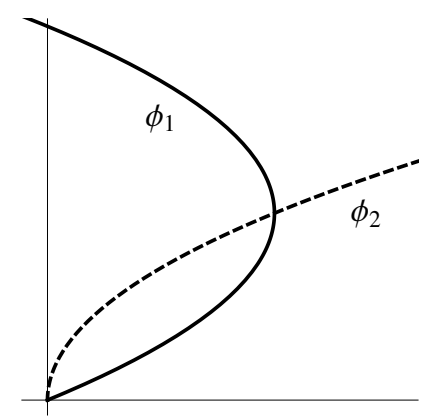

(c)

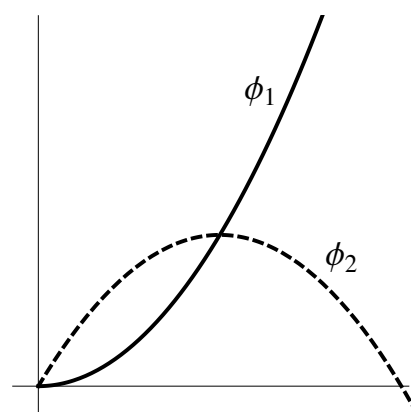

(b)

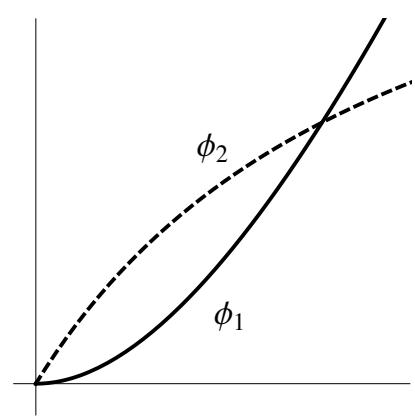

(d)

Figure 17: The parametric curves $\phi_{1}$ and $\phi_{2}$ are plotted for various situations described in Theorem 4. In (a) conditions (A), (B1) and (B2) are satisfied, in (b) (A) and (B2) are satisfied, in (c) (A) and (B1) are satisfied and in (d) (A) and (B3) are satisfied.

The curves $\mathcal{C}_{1}$ and $\mathcal{C}_{2}$ intersect if and only if $\phi_{1}$ and $\phi_{2}$ intersect. We need the following derivatives:

$$
\begin{aligned}
& \left.\frac{\mathrm{d} y_{1}}{\mathrm{~d} x_{1}}\right|_{t}=\frac{m_{21} f_{1}^{\prime}(t)}{1-m_{11} f_{1}^{\prime}(t)} \quad \text { and }\left.\quad \frac{\mathrm{d} y_{2}}{\mathrm{~d} x_{2}}\right|_{t}=\frac{1-m_{22} f_{2}^{\prime}(t)}{m_{12} f_{2}^{\prime}(t)} \\
& \left.\frac{\mathrm{d}^{2} x_{1}}{\mathrm{~d} y_{1}^{2}}\right|_{t}=-\frac{f_{1}^{\prime \prime}(t)}{m_{21}^{2}\left(f_{1}^{\prime}(t)\right)^{3}} \quad \text { and }\left.\quad \frac{\mathrm{d}^{2} y_{2}}{\mathrm{~d} x_{2}^{2}}\right|_{t}=-\frac{f_{2}^{\prime \prime}(t)}{m_{12}^{2}\left(f_{2}^{\prime}(t)\right)^{3}} .
\end{aligned}
$$

Note that the geometric interpretations of conditions (A), (B1), (B2), and (B3) from Theorem 4 are as follows:

(A) $\left.\frac{d y_{1}}{d x_{1}}\right|_{0},\left.\frac{d y_{2}}{d x_{2}}\right|_{0}>0$ and $\left.\frac{d y_{2}}{d x_{2}}\right|_{0}>\left.\frac{d y_{1}}{d x_{1}}\right|_{0}$

(B1) There exists $t^{*}>0$ such that $\left.\frac{d x_{1}}{d t}\right|_{t^{*}}=0$ 
(B2) There exists $t^{*}>0$ such that $\left.\frac{d y_{2}}{d t}\right|_{t^{*}}=0$

(B3) $\lim _{t \rightarrow \infty} \frac{d y_{2}}{d x_{2}}<\lim _{t \rightarrow \infty} \frac{d y_{1}}{d x_{1}}$.

Under the hypotheses imposed on $f_{1}, f_{2}$, it is clear from (18) that

$$
\frac{\mathrm{d}^{2} x_{1}}{\mathrm{~d} y_{1}^{2}}<0 \quad \text { and } \quad \frac{\mathrm{d}^{2} y_{2}}{\mathrm{~d} x_{2}^{2}}<0 \quad \text { for all } t \geq 0
$$

Let $\mathcal{Q}_{1}$ be the standard first quadrant. We claim that if $m_{11} f_{1}^{\prime}(0) \geq 1$, then $\left|\phi_{1}\right| \cap\left(\mathcal{Q}_{1} \backslash\{(0,0)\}\right)=\emptyset$. Similarly, if $m_{22} f_{2}^{\prime}(0) \geq 1$, then $\left|\phi_{2}\right| \cap\left(\mathcal{Q}_{1} \backslash\{(0,0)\}\right)=\emptyset$. Suppose that $m_{11} f_{1}^{\prime}(0)>1$, then clearly $\left.\frac{\mathrm{d} y_{1}}{\mathrm{~d} x_{1}}\right|_{0}<0$. Since $f_{1}$ is increasing, (16) implies $\left.\frac{\mathrm{d} x_{1}}{\mathrm{~d} t}\right|_{0}<0$ and $\frac{d y_{1}}{d t}>0$ for all $t$. Combining this with the fact that $\frac{\mathrm{d}^{2} x_{1}}{\mathrm{~d} y_{1}^{2}}<0$, we can conclude $\frac{\mathrm{d} x_{1}}{\mathrm{~d} t}<0$ for all $t \geq 0$. Hence, $\left|\phi_{1}\right| \cap\left(\mathcal{Q}_{1} \backslash\{(0,0)\}\right)=\emptyset$. If $m_{11} f_{1}^{\prime}(0)=1$, the same conclusion follows from (19). A similar argument can be used if $m_{22} f_{2}^{\prime}(0) \geq 1$, thus completing the proof of the claim.

$(\Rightarrow)$ Let $\tilde{T}$ have a positive fixed point $\left(t_{1}, t_{2}\right) \in(0, \infty) \times(0, \infty)$. Suppose that (A) is not satisfied. This implies that $m_{11} f_{1}^{\prime}(0) \geq 1, m_{22} f_{2}^{\prime}(0) \geq 1$, or

$$
\frac{1-m_{22} f_{2}^{\prime}(0)}{m_{12} f_{2}^{\prime}(0)} \leq \frac{m_{21} f_{1}^{\prime}(0)}{1-m_{11} f_{1}^{\prime}(0)}
$$

If $m_{11} f_{1}^{\prime}(0) \geq 1$ or $m_{22} f_{2}^{\prime}(0) \geq 1$, then by the above claim, $\left|\phi_{1}\right| \cap\left(\mathcal{Q}_{1} \backslash\{(0,0)\}\right)=\emptyset$ or $\left|\phi_{2}\right| \cap\left(\mathcal{Q}_{1} \backslash\{(0,0)\}\right)=\emptyset$, which means $\phi_{1}$ and $\phi_{2}$ do not have a positive intersection point, a contradiction. If (20) holds, then $\left.\frac{\mathrm{d} y_{2}}{\mathrm{~d} x_{2}}\right|_{0} \leq\left.\frac{\mathrm{d} y_{1}}{\mathrm{~d} x_{1}}\right|_{0}$ and it follows from (19) that a positive intersection point cannot exist, thus, (A) holds.

Now, suppose that (A) is satisfied and that (B1), (B2) and (B3) are not satisfied. From (A),

$$
\left.\frac{\mathrm{d} y_{1}}{\mathrm{~d} x_{1}}\right|_{0}<\left.\frac{\mathrm{d} y_{2}}{\mathrm{~d} x_{2}}\right|_{0}
$$

Since (B1) and (B2) are not satisfied, then $\frac{\mathrm{d} y_{1}}{\mathrm{~d} x_{1}}, \frac{\mathrm{d} y_{1}}{\mathrm{~d} x_{1}}>0$ for all $t \geq 0$. From (19), (21) and the fact that (B3) is not satisfied, it follows that $\phi_{1}$ and $\phi_{2}$ do not have a positive intersection, a contradiction. We conclude that if $\tilde{T}$ has a unique positive 
fixed point $\left(t_{1}, t_{2}\right) \in(0, \infty) \times(0, \infty)$, then $(\mathrm{A})$ is satisfied along with at least one of (B1), (B2) and (B3).

$(\Leftarrow)$ Suppose that $(\mathrm{A})$ is satisfied, in which case $(21)$ holds. We consider three separate cases when (B1), (B2) and (B3) are each satisfied and prove that there exists a fixed point $\left(t_{1}, t_{2}\right) \in(0, \infty) \times(0, \infty)$ for the map $\tilde{T}$. If (B1) is satisfied, then there exists $t^{*}>0$ such that $\left.\frac{d x_{1}}{d t}\right|_{t^{*}}=0$. Since $f_{1}$ is increasing and concave,

$$
m_{11} f_{1}^{\prime}(t)>1 \text { for all } t>t^{*}
$$

Combining (22) with (17) and (19), there must exist $t_{1}>t^{*}$ such that $x_{1}\left(t_{1}\right)=0$ and $y_{1}(t)>0$. Consequently, it follows from (21) that the parametric curves $\phi_{1}$ and $\phi_{2}$ have a positive intersection and thus $\tilde{T}$ has a positive fixed point. The case involving (B2) is similar.

Finally, suppose that (B3) is satisfied, which implies that (B1) and (B2) are not satisfied. In other words,

$$
\frac{\mathrm{d} y_{1}}{\mathrm{~d} x_{1}}, \frac{\mathrm{d} y_{2}}{\mathrm{~d} x_{2}}>0 \text { for all } t \geq 0
$$

From (21), (23) and (B3), the parametric curves $\phi_{1}$ and $\phi_{2}$ have a positive intersection point, hence $\tilde{T}$ has a fixed point in $(0, \infty) \times(0, \infty)$. If $\tilde{T}$ has two or more positive fixed points, it can be checked (with similar arguments) that a contradiction occurs. Hence if a positive fixed point exists, it is unique.

Now, from $(\mathrm{A}),\left.\frac{d y_{2}}{d x_{2}}\right|_{0} \neq\left.\frac{d y_{1}}{d x_{1}}\right|_{0}$, which implies that the curves $\phi_{1}$ and $\phi_{2}$ do not have a tangential contact point at the origin. Similarly, based on the curvature of $\phi_{1}$ and $\phi_{2}$ and conditions (B1), (B2), and (B3), $\phi_{1}$ and $\phi_{2}$ do not have a tangential contact point at $\left(t_{1}, t_{2}\right)$. It follows that the origin and $\left(t_{1}, t_{2}\right)$ are hyperbolic by a result stated in [17].

Theorem 5 establishes necessary and sufficient conditions for the existence of a unique positive fixed point of $\tilde{T}$ when $f_{1}, f_{2}$ are strictly increasing and concave. 
Theorem 5 Let $M>0$ and suppose that $f_{1}, f_{2}$ are twice differentiable, strictly increasing, and concave. Consider the following conditions:

(A) The limits $L_{1}$ and $L_{2}$ exist and $L_{2}>L_{1}$, where

$$
L_{1}:=\lim _{t \rightarrow \infty}\left[\frac{m_{21} f_{1}^{\prime}(t)}{1-m_{11} f_{1}^{\prime}(t)}\right] \text { and } L_{2}:=\lim _{t \rightarrow \infty}\left[\frac{1-m_{22} f_{2}^{\prime}(t)}{m_{12} f_{2}^{\prime}(t)}\right] \text {. }
$$

(B1) $m_{11} f_{1}^{\prime}(0) \leq 1$.

(B2) $m_{11} f_{1}^{\prime}(0)>1$ and there exists $t^{*}>0$ such that $m_{11} f_{1}^{\prime}\left(t^{*}\right)=1$.

(C1) $m_{22} f_{2}^{\prime}(0) \leq 1$

(C2) $m_{22} f_{2}^{\prime}(0)>1$ and there exists $t^{*}>0$ such that $m_{22} f_{2}^{\prime}\left(t^{*}\right)=1$.

(D) $\max \left\{m_{11} f_{1}^{\prime}(0), m_{11} f_{2}^{\prime}(0)\right\} \leq 1$ and

$$
\left(1-m_{22} f_{2}^{\prime}(0)\right)\left(1-m_{11} f_{1}^{\prime}(0)\right)<m_{21} m_{12} f_{1}^{\prime}(0) f_{2}^{\prime}(0)
$$

The map $\tilde{T}$ has a positive fixed point $\left(t_{1}, t_{2}\right)$ if and only if $(\mathrm{A})$ is satisfied along with (i) (B1) and (C2), (ii) (B2) and (C1), (iii) (B2) and (C2) or (ii) (D). When a positive fixed point $\left(t_{1}, t_{2}\right)$ exists, it is unique. Furthermore, in this case, $\left(t_{1}, t_{2}\right)$ is hyperbolic and if $\left(1-m_{22} f_{2}^{\prime}(0)\right)\left(1-m_{11} f_{1}^{\prime}(0)\right) \neq m_{21} m_{12} f_{1}^{\prime}(0) f_{2}^{\prime}(0)$, then the origin is hyperbolic.

Remark 3 It follows from (13) that the limit $L_{2}$ exists and the inequality in (D) is well defined. Also, if (B1), (B2), or (D) is satisfied, then based on the monotonicity and concavity of $f_{1}$, it can be shown that there exists $t^{*}>0$ such that for all $t>t^{*}$, $m_{11} f_{1}^{\prime}(t)<1$, which implies that the limit $L_{1}$ exists.

Proof. The proof of Theorem 5 is similar to that of Theorem 4 and is omitted here. 


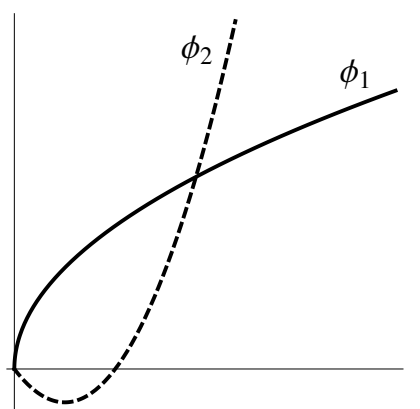

(a)

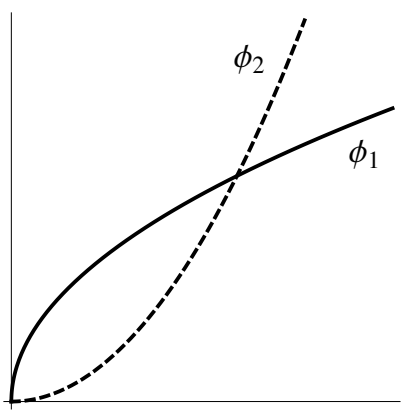

(c)

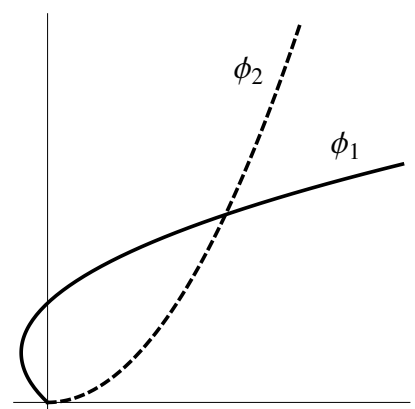

(b)

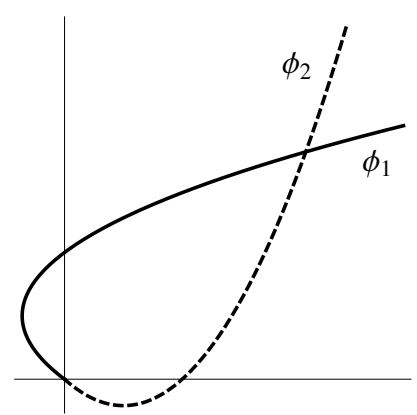

(d)

Figure 18: The parametric curves $\phi_{1}$ and $\phi_{2}$ are plotted for different cases described in Theorem 5. In figure (a) conditions (B1) and (C2) are satisfied, in (b) (B2) and (C1) are satisfied, in (c) (D) is satisfied and in (d) (B2) and (C2) are satisfied. Note that hypothesis (A) is satisfied in all cases.

\subsubsection{Stability of the Origin}

Theorems 4 and 5 from Section 4.4 .1 provide necessary and sufficient conditions for the map $\tilde{T}$ from (12) to have a unique positive fixed point under the conditions set forth in Cases 1 and 2 from (14). In these special cases, more can be said about the stability of the origin as a fixed point of the map $\tilde{T}$. The Jacobian matrix associated with $\tilde{T}$ is

$$
J_{\tilde{T}}\left(\begin{array}{l}
u \\
v
\end{array}\right)=\left(\begin{array}{ll}
m_{11} f_{1}^{\prime}(u) & m_{12} f_{2}^{\prime}(v) \\
m_{21} f_{1}^{\prime}(u) & m_{22} f_{2}^{\prime}(v)
\end{array}\right)
$$

When $f_{1}^{\prime}(t), f_{2}^{\prime}(t) \geq 0$ for all $t \geq 0$, all entries of $J_{\tilde{T}}$ are nonnegative on $\mathbb{R}_{+}^{2}$ and thus the map $\tilde{T}$ is monotone (i.e. cooperative). Evaluating the Jacobian matrix 
at the fixed point $(0,0)$, we have

$$
J_{\tilde{T}}\left(\begin{array}{l}
0 \\
0
\end{array}\right)=\left(\begin{array}{ll}
m_{11} f_{1}^{\prime}(0) & m_{12} f_{2}^{\prime}(0) \\
m_{21} f_{1}^{\prime}(0) & m_{22} f_{2}^{\prime}(0)
\end{array}\right),
$$

from which it follows that the eigenvalues associated with $J_{\tilde{T}}(0,0)$ are the roots of the characteristic equation

$$
\lambda^{2}-\left(m_{11} f_{1}^{\prime}(0)+m_{22} f_{2}^{\prime}(0)\right) \lambda+f_{1}^{\prime}(0) f_{2}^{\prime}(0)\left(m_{11} m_{22}-m_{12} m_{21}\right)=0 .
$$

In Case 1 from (14), if $\tilde{T}$ has a unique positive fixed point $\overline{\mathrm{x}}$, then it is shown that $\overline{\mathrm{x}}$ is a repeller or a saddle point and that the fixed point at the origin is locally asymptotically stable. In Case 2, if $\tilde{T}$ has a unique positive fixed point $\overline{\mathrm{x}}$, then under one additional assumption (to rule out nonhyperbolic cases) it is verified that $\overline{\mathrm{x}}$ is locally asymptotically stable and that the fixed point at the origin is unstable. These statements are formally presented and proven in Theorems 6 and 7.

Theorem 6 Suppose that $f_{1}, f_{2}$ are twice differentiable, strictly increasing, and convex. If the map $\tilde{T}$ has a unique positive fixed point $\overline{\mathrm{x}}$, then $\overline{\mathrm{x}}$ is a repeller or a saddle point and the fixed point at the origin is locally asymptotically stable.

Proof. From (13), $f_{1}^{\prime}(0), f_{2}^{\prime}(0)>0$. By the Schur-Cohn criterion, to prove that the fixed point at the origin is locally asymptotically stable, it is sufficient to verify that

$$
\left|\operatorname{tr} J_{\tilde{T}}(0)\right|<1+\operatorname{det} J_{\tilde{T}}(0)<2 .
$$

In this case, condition (24) is equivalent to

$$
\frac{m_{11}}{f_{2}^{\prime}(0)}+\frac{m_{22}}{f_{1}^{\prime}(0)}-\frac{1}{f_{1}^{\prime}(0) f_{2}^{\prime}(0)}<\operatorname{det} M<\frac{1}{f_{1}^{\prime}(0) f_{2}^{\prime}(0)}
$$

where $\operatorname{det} M=m_{11} m_{22}-m_{12} m_{21}$. Since $f_{1}, f_{2}$ are strictly increasing and convex and $\tilde{T}$ have as unique positive fixed point $\overline{\mathrm{x}}$, then Theorem 4 guarantees

$$
\max \left\{m_{11} f_{1}^{\prime}(0), m_{22} f_{2}^{\prime}(0)\right\}<1 \text { and } \frac{1-m_{22} f_{2}^{\prime}(0)}{m_{12} f_{2}^{\prime}(0)}>\frac{m_{21} f_{1}^{\prime}(0)}{1-m_{11} f_{1}^{\prime}(0)}
$$


It follows from the first condition in (26) that

$$
m_{11} m_{22}<\frac{1}{f_{1}^{\prime}(0) f_{2}^{\prime}(0)}
$$

Consequently, since $\operatorname{det} M<m_{11} m_{22}$ we have $\operatorname{det} M<\frac{1}{f_{1}^{\prime}(0) f_{2}^{\prime}(0)}$. From the second condition in $(26)$, it follows that $\operatorname{det} M>\frac{m_{11}}{f_{2}^{\prime}(0)}+\frac{m_{22}}{f_{1}^{\prime}(0)}-\frac{1}{f_{1}^{\prime}(0) f_{2}^{\prime}(0)}$ and thus (25) holds and the fixed point at the origin is locally asymptotically stable.

By monotonicity of $\tilde{T}$, the order interval $\llbracket 0, \overline{\mathrm{x}} \rrbracket$ is $\tilde{T}$-invariant and, from a theorem presented in $[18]$, the interior of the order interval $\llbracket 0, \bar{x} \rrbracket$ is a subset of the basin of attraction of 0 or $\overline{\mathrm{x}}$. Since 0 is locally asymptotically stable, then $\llbracket 0, \overline{\mathrm{x}} \rrbracket$ is a subset of the basin of attraction of 0 . Consequently, $\overline{\mathrm{x}}$ is unstable. Theorem 4 guarantees that $\overline{\mathrm{x}}$ is hyperbolic and thus it is either a repeller or a saddle point.

The next theorem treats Case 2 from (14), where $f_{1}, f_{2}$ are twice differentiable, strictly increasing, and concave. Note that the assumption in (27) is included to ensure that the origin is not nonhyperbolic.

Theorem 7 Suppose that $f_{1}, f_{2}$ are twice differentiable, strictly increasing, and concave. Additionally assume that

$$
\left(1-m_{22} f_{2}^{\prime}(0)\right)\left(1-m_{11} f_{1}^{\prime}(0)\right) \neq m_{21} m_{12} f_{1}^{\prime}(0) f_{2}^{\prime}(0)
$$

If the map $\tilde{T}$ has a unique positive fixed point $\overline{\mathrm{x}}$, then $\overline{\mathrm{x}}$ is locally asymptotically stable and the fixed point at the origin is unstable.

Proof. From (13), $f_{1}^{\prime}(0), f_{2}^{\prime}(0)>0$. Suppose that $\tilde{T}$ has a unique positive fixed point. By the Schur-Cohn criterion, to prove that the fixed point at the origin is not stable, it is sufficient to verify that

$$
\left|\operatorname{tr} J_{\tilde{T}}(0)\right|>1+\operatorname{det} J_{\tilde{T}}(0) \quad \text { or } \quad \operatorname{det} J_{\tilde{T}}(0)>1
$$


Condition (28) is equivalent to

$$
\operatorname{det} M<\frac{m_{11}}{f_{2}^{\prime}(0)}+\frac{m_{22}}{f_{1}^{\prime}(0)}-\frac{1}{f_{1}^{\prime}(0) f_{2}^{\prime}(0)} \quad \text { or } \quad \operatorname{det} M>\frac{1}{f_{1}^{\prime}(0) f_{2}^{\prime}(0)}
$$

Since $f_{1}, f_{2}$ are strictly increasing and concave and $\tilde{T}$ has a unique positive fixed point, then Theorem 5 guarantees that one of the cases (i) through (iv) from the statement of the theorem is satisfied. We consider each case separately to verify that (29) holds.

If (i) holds then $m_{11} \leq \frac{1}{f_{1}^{\prime}(0)}$ and $m_{22}>\frac{1}{f_{2}^{\prime}(0)}$. Therefore,

$$
m_{22}\left(\frac{1}{f_{1}^{\prime}(0)}-m_{11}\right) \geq \frac{1}{f_{2}^{\prime}(0)}\left(\frac{1}{f_{1}^{\prime}(0)}-m_{11}\right),
$$

which implies that $m_{11} m_{22} \leq \frac{m_{11}}{f_{1}^{\prime}(0)}+\frac{m_{22}}{f_{2}^{\prime}(0)}-\frac{1}{f_{1}^{\prime}(0) f_{2}^{\prime}(0)}$. Since $\operatorname{det} M<m_{11} m_{22}$, it follows that the first condition in (29) is satisfied. A similar argument can be used if (ii) holds. If (iii) holds then $m_{11}>\frac{1}{f_{1}^{\prime}(0)}$ and $m_{22}>\frac{1}{f_{2}^{\prime}(0)}$, in which case

$$
\frac{m_{11}}{f_{2}^{\prime}(0)}+\frac{m_{22}}{f_{1}^{\prime}(0)}>\frac{2}{f_{1}^{\prime}(0) f_{2}^{\prime}(0)}
$$

Proceed by contradiction and assume that (29) is not satisfied. That is, assume

$$
\frac{m_{11}}{f_{2}^{\prime}(0)}+\frac{m_{22}}{f_{1}^{\prime}(0)}-\frac{1}{f_{1}^{\prime}(0) f_{2}^{\prime}(0)}<\operatorname{det} M<\frac{1}{f_{1}^{\prime}(0) f_{2}^{\prime}(0)} .
$$

It follows that $\frac{m_{11}}{f_{2}^{\prime}(0)}+\frac{m_{22}}{f_{1}^{\prime}(0)}<\frac{2}{f_{1}^{\prime}(0) f_{2}^{\prime}(0)}$, a contradiction to (30). Finally, if (iv) holds, then $\max \left\{m_{11} f_{1}^{\prime}(0), m_{22} f_{2}^{\prime}(0)\right\}<1$ and

$$
\frac{1-m_{22} f_{2}^{\prime}(0)}{m_{12} f_{2}^{\prime}(0)}<\frac{m_{21} f_{1}^{\prime}(0)}{1-m_{11} f_{1}^{\prime}(0)}
$$

The inequality in (31) implies that the first condition in (29) is satisfied. Therefore, the origin is not stable. By Theorem 5 and (27), the origin is hyperbolic, which implies that it is unstable. As a result, the interior of the order interval $\llbracket 0, \overline{\mathrm{x}} \rrbracket$ is a subset of the basin of attraction of $\overline{\mathrm{x}}[18]$.

Since $f_{1}, f_{2} \in C^{(1)}$, the Taylor expansion of $\tilde{T}$ about $\overline{\mathrm{x}}$ is

$$
\tilde{T}(\mathrm{y})=\tilde{T}(\overline{\mathrm{x}})+\rho\left(J_{\tilde{T}}(\overline{\mathrm{x}})\right) \mathrm{y}+o(|\mathrm{y}|) .
$$


The irreducibility of $M$, along with (13), implies that $J_{\tilde{T}}(\overline{\mathrm{x}})$ is irreducible. By the Perron-Frobenius theorem, $\rho^{*}=\rho\left(J_{\tilde{T}}(\overline{\mathrm{x}})\right)$ is a positive eigenvalue of $J_{\tilde{T}}(\overline{\mathrm{x}})$ associated with a positive eigenvector $\mathrm{v}$. That is,

$$
J_{\tilde{T}}(\overline{\mathrm{x}}) \mathrm{v}=\rho^{*} \mathrm{v}
$$

For $\alpha<0$, take $\mathrm{y}=\overline{\mathrm{x}}+\alpha \mathrm{v}$. Consequently,

$$
\begin{aligned}
\tilde{T}(\overline{\mathrm{x}}+\alpha \mathrm{v}) & =\tilde{T}(\overline{\mathrm{x}})+J_{\tilde{T}}(\overline{\mathrm{x}}) \alpha \mathrm{v}+o(|\alpha \mathrm{v}|) \\
& =\overline{\mathrm{x}}+\rho^{*} \alpha \mathrm{v}+o(|\alpha|)
\end{aligned}
$$

Therefore

$$
\tilde{T}(\mathrm{y})-\mathrm{y}=\alpha\left(\left(\rho^{*}-1\right) \mathrm{v}+o(1)\right)
$$

Since the interior of the order interval $\llbracket 0, \overline{\mathrm{x}} \rrbracket$ is a subset of the basin of attraction of $\overline{\mathrm{x}}$, for $\alpha<0$ chosen sufficient close to zero, the left hand side of (32) is positive, which implies that $\rho^{*}<1$. Hence $\overline{\mathrm{x}}$ is locally asymptotically stable.

The above results provide a strong connection between the existence of a positive fixed point and the stability of the origin when $f_{1}, f_{2}$ satisfy the conditions set forth in (14). A natural extension is to investigate the stability of the origin in these same cases when it is assumed that a positive fixed point does not exist. This will be investigated in the future.

\subsubsection{Examples}

To illustrate Theorems 4, 5, 6, and 7 presented in Sections 4.4.1 and 4.4.2, two specific examples are provided in the current section. Example 1 considers systems of the form (I) with $k=2$, where the functions are of Beverton-Holt type from (4). Example 2 considers quadratic fractional type functions. 
Example 1 Consider the system of difference equations

$$
\begin{aligned}
& x_{n+1}=\frac{m_{11} x_{n}}{\delta_{1}+x_{n}}+\frac{m_{12} y_{n}}{\delta_{2}+y_{n}}, \\
& y_{n+1}=\frac{m_{21} x_{n}}{\delta_{1}+x_{n}}+\frac{m_{22} y_{n}}{\delta_{2}+y_{n}}, \quad n=0,1,2, \ldots,
\end{aligned}
$$

where $m_{11}, m_{12}, m_{21}, m_{22}, \delta_{1}, \delta_{2}>0$ and the initial condition $\left(x_{0}, y_{0}\right) \in(0, \infty) \times$ $(0, \infty)$. The map $T_{1}$ associated with system $(33)$ is

$$
T_{1}\left(\begin{array}{l}
u \\
v
\end{array}\right)=\left(\begin{array}{l}
\frac{m_{11} u}{\delta_{1}+u}+\frac{m_{12} v}{\delta_{2}+v} \\
\frac{m_{21} u}{\delta_{1}+u}+\frac{m_{22} v}{\delta_{2}+v}
\end{array}\right)
$$

and has the form given in (12), where $f_{\ell}(t)=\frac{t}{\delta_{\ell}+t}$ for $\ell=1,2$. A simple calculation shows that $f_{1}, f_{2}$ are strictly increasing and concave. Therefore, Theorem 5 can be applied to determine necessary and sufficient conditions for which a unique positive fixed point exists for the map $T_{1}$. When it is known that $T_{1}$ has a unique positive fixed point, one can apply Theorem 7 and the general results from Section 4.3 to determine the global character of system (33). It is shown that there are only two dynamical scenarios: Either there exists a unique positive fixed point $\overline{\mathrm{x}}$ that is a global attractor or there are no positive fixed points and the origin is a global attractor. This result is presented as Proposition 1. Note that (35) is included to ensure that the origin is not nonhyperbolic.

Proposition 1 Suppose that

$$
\left(\delta_{2}-m_{22}\right)\left(\delta_{1}-m_{11}\right) \neq m_{21} m_{12}
$$

The map $T_{1}$ in (34) has a positive fixed point $\overline{\mathrm{x}}$ if and only if at least one of the following conditions is satisfied:

(H1) $m_{11} \leq \delta_{1}$ and $m_{22}>\delta_{2}$ or $m_{11}>\delta_{1}$ and $m_{22} \leq \delta_{2}$.

(H2) $m_{11}>\delta_{1}$ and $m_{22}>\delta_{2}$. 


$$
m_{11} \leq \delta_{1}, m_{22} \leq \delta_{2}, \text { and }\left(\delta_{2}-m_{22}\right)\left(\delta_{1}-m_{11}\right)<m_{21} m_{12}
$$

When it exists, $\overline{\mathrm{x}}$ is unique and a global attractor on $\operatorname{int}\left(\mathbb{R}_{+}^{2}\right)$. When there does not exist a positive fixed point, the fixed point at the origin is a global attractor.

Proof. In this case,

$$
\begin{gathered}
\lim _{t \rightarrow \infty}\left[\frac{1-m_{22} f_{2}^{\prime}(t)}{m_{12} f_{2}^{\prime}(t)}\right]=\lim _{t \rightarrow \infty}\left[\frac{\left(\delta_{2}+t\right)^{2}-\delta_{2} m_{22}}{\delta_{2} m_{12}}\right]=+\infty, \\
\lim _{t \rightarrow \infty}\left[\frac{m_{21} f_{1}^{\prime}(t)}{1-m_{11} f_{1}^{\prime}(t)}\right]=\lim _{t \rightarrow \infty}\left[\frac{\delta_{1} m_{21}}{\left(\delta_{1}+t\right)^{2}-\delta_{1} m_{11}}\right]=0,
\end{gathered}
$$

which implies that (A) of Theorem 5 is satisfied. Now, assume (H1) is satisfied. First consider the case when $m_{11} \leq \delta_{1}$ and $m_{22}>\delta_{2}$. This implies that (B1) holds. Furthermore, a simple calculation shows that there exists $t^{*}=\sqrt{m_{22} \delta_{2}}-\delta_{2}>0$ such that $m_{22} f_{2}^{\prime}\left(t^{*}\right)=1$ and thus (C2) holds. Using similar logic, $m_{11}>\delta_{1}$ and $m_{22} \leq \delta_{2}$ are equivalent to (B2), (C1) from Theorem 5. Similarly, (H2) is equivalent to (B2), (C2) and (H3) is equivalent to (D). The existence and uniqueness of a positive fixed point $\overline{\mathrm{x}}$ follows from Theorem 5 .

Since $\left(\delta_{2}-m_{22}\right)\left(\delta_{1}-m_{11}\right) \neq m_{21} m_{12}$, by Theorem 5 and 7 the origin is hyperbolic and unstable. Hence, $\rho\left(J_{T_{1}}(0)\right)>1$. Also, $M=\left(m_{i, j}\right)$ is irreducible and

$$
\begin{aligned}
\overline{f^{\prime}(\infty)} & =\max _{\ell}\left\{\limsup _{t \rightarrow \infty} \frac{f_{\ell}(t)}{t}\right\} \\
& =\max _{\ell}\left\{\limsup _{t \rightarrow \infty} \frac{1}{\delta_{\ell}+t}\right\}=0 .
\end{aligned}
$$

Consequently, $\overline{\mathrm{x}}$ is a global attractor on $\operatorname{int}\left(\mathbb{R}_{+}^{2}\right)$ by Theorem 3. Now, if $\overline{\mathrm{x}}$ does not exist then (H1), (H2), and (H3) are not satisfied. This immediately implies that $m_{11} \leq \delta_{1}, m_{22} \leq \delta_{2}$ and $\left(\delta_{2}-m_{22}\right)\left(\delta_{1}-m_{11}\right)>m_{21} m_{12}$. We will verify that the origin is locally asymptotically stable by applying Shur-Cohn criterion to the Jacobian of the map evaluated at 0 . That is, we will verify conditions (25), given 
in this example as

$$
\delta_{2} m_{11}+\delta_{1} m_{22}-\delta_{1} \delta_{2}<\operatorname{det} M<\delta_{1} \delta_{2}
$$

Since $m_{11} \leq \delta_{1}, m_{22} \leq \delta_{2}$ then

$$
\operatorname{det} M<m_{11} m_{22} \leq \delta_{1} \delta_{2}
$$

which verifies the second inequality from (37). The first inequality from (37) then follows directly from $\left(\delta_{2}-m_{22}\right)\left(\delta_{1}-m_{11}\right)>m_{21} m_{12}$. Consequently, the origin is locally asymptotically stable.

Using (36), Lemma 1 guarantees that there exists a point $\mathrm{y} \in(0, \infty) \times(0, \infty)$, with $\|\mathrm{y}\|$ as large as we like, such that $T_{1}(\mathrm{y}) \prec \mathrm{y}$. Therefore, from Theorem 1 , the order interval $\llbracket 0, y \rrbracket$ is invariant under $T_{1}$. With no other interior fixed points, a result from [18] ensures that the interior of $\llbracket 0, y \rrbracket$ is a subset of the basin of attraction of the origin. Since y can be chosen as large as we like, the origin is a global attractor.

It is worth mentioning that the global dynamics of (33) can be completely characterized using general theory of two-dimensional cooperative systems of difference equations. The example is presented only to illustrate the main theorems. The next example illustrates both Theorems 4 and 5 .

Example 2: Consider the system of difference equations

$$
\begin{aligned}
& x_{n+1}=\frac{m_{11} x_{n}\left(x_{n}+\alpha_{1}\right)}{x_{n}+\beta_{1}}+\frac{m_{12} y_{n}\left(y_{n}+\alpha_{2}\right)}{y_{n}+\beta_{2}}, \\
& y_{n+1}=\frac{m_{21} x_{n}\left(x_{n}+\alpha_{1}\right)}{x_{n}+\beta_{1}}+\frac{m_{22} y_{n}\left(y_{n}+\alpha_{2}\right)}{y_{n}+\beta_{2}}, \quad n=0,1,2, \ldots,
\end{aligned}
$$

where all parameters are taken to be strictly positive and the initial condition $\left(x_{0}, y_{0}\right) \in(0, \infty) \times(0, \infty)$. The map $T_{2}$ associated with $(38)$ is

$$
T_{2}\left(\begin{array}{l}
u \\
v
\end{array}\right)=\left(\begin{array}{l}
\frac{m_{11} u\left(u+\alpha_{1}\right)}{u+\beta_{1}}+\frac{m_{12} v\left(v+\alpha_{2}\right)}{v+\beta_{2}} \\
\frac{m_{21} u\left(u+\alpha_{1}\right)}{u+\beta_{1}}+\frac{m_{22} v\left(v+\alpha_{2}\right)}{v+\beta_{2}}
\end{array}\right),
$$


and has the form (12), where $f_{\ell}=\frac{t\left(t+\alpha_{\ell}\right)}{t+\beta_{\ell}}$ for $\ell=1,2$. It can be easily verified that $f_{1}, f_{2}$ are strictly increasing functions. Also notice

$$
\frac{d^{2} f_{\ell}}{d t^{2}}=\frac{2 \beta_{\ell}\left(\beta_{\ell}-\alpha_{\ell}\right)}{\left(t+\beta_{\ell}\right)^{3}}
$$

and thus for $\ell=1,2$ we have the following:

$$
\begin{aligned}
& f_{\ell} \text { is convex if } \beta_{\ell}-\alpha_{\ell}>0 \\
& f_{\ell} \text { is concave if } \beta_{\ell}-\alpha_{\ell}<0
\end{aligned}
$$

Theorem 4 and 5 can be applied to determine necessary and sufficient conditions for which $T_{2}$ has a unique positive fixed point in the case when the $f_{\ell}$ 's are either both convex or both concave. When it is known that $T_{2}$ has a unique positive fixed point, one can apply the general results from Section 4.3 to establish the global character of (38). The case when $f_{1}, f_{2}$ are both convex is treated first in Proposition 2.

Proposition 2 Let $\beta_{\ell}-\alpha_{\ell}>0$ for $\ell=1,2$. The map $T_{2}$ has a positive fixed point $\overline{\mathrm{x}}$ if and only if

$$
m_{11} \in\left(0, \frac{\beta_{1}}{\alpha_{1}}\right), \quad m_{22} \in\left(0, \frac{\beta_{2}}{\alpha_{2}}\right), \quad \frac{\beta_{2}-m_{22} \alpha_{2}}{m_{12} \alpha_{2}}>\frac{m_{21} \alpha_{1}}{\beta_{1}-m_{11} \alpha_{1}},
$$

and one of the following conditions is satisfied:

(i) $\max \left\{m_{11}, m_{22}\right\}>1$.

(ii) $m_{11}, m_{22} \neq 1$ and $\frac{1-m_{22}}{m_{12}}<\frac{m_{21}}{1-m_{11}}$.

Furthermore, when $\overline{\mathrm{x}}$ exists, it is unique and is a saddle point or a repeller.

Proof. Note that in this case $f_{1}, f_{2}$ are convex by (40). Therefore, Theorem 4 can be applied. The conditions in (42) are equivalent to condition (A). If $\max \left\{m_{11}, m_{22}\right\}>1$, then either $m_{11}>1$ or $m_{22}>1$. If $m_{11}>1$, it can be 
easily verified (using $m_{11}<\frac{\beta_{1}}{\alpha_{1}}$ and $\beta_{1}-\alpha_{1}>0$ ) that $m_{11} f_{1}^{\prime}(t)=1$ has a positive, real solution given by

$$
t^{*}=\frac{\beta_{1}\left(1-m_{11}\right)+\sqrt{\beta_{1} m_{11}\left(\beta_{1}-\alpha_{1}\right)\left(m_{11}-1\right)}}{m_{11}-1}
$$

and thus (B2) is satisfied. Similarly, $m_{22}>1$ is equivalent to (B1). Furthermore,

$$
\begin{aligned}
& \lim _{t \rightarrow \infty}\left[\frac{1-m_{22} f_{2}^{\prime}(t)}{m_{12} f_{2}^{\prime}(t)}\right]=\frac{1-m_{22}}{m_{12}}, \\
& \lim _{t \rightarrow \infty}\left[\frac{m_{21} f_{1}^{\prime}(t)}{1-m_{11} f_{1}^{\prime}(t)}\right]=\frac{m_{21}}{1-m_{11}},
\end{aligned}
$$

and thus the condition in (ii) is equivalent to (B3). Consequently, the existence and uniqueness of $\overline{\mathrm{x}}$ follows from Theorem 4 and the instability of $\overline{\mathrm{x}}$ follows from Theorem 6 .

The next result, Proposition 3, treats the case when $f_{1}, f_{2}$ are concave.

Proposition 3 Let $\beta_{\ell}-\alpha_{\ell}<0$ for $\ell=1,2$. The map $T_{2}$ has a positive fixed point $\overline{\mathrm{x}}$ if and only if

$$
\frac{1-m_{22}}{m_{12}}>\frac{m_{21}}{1-m_{11}}
$$

and any of the following conditions are satisfied:

(i) $m_{11} \in\left(0, \frac{\beta_{1}}{\alpha_{1}}\right]$ and $m_{22} \in\left(\frac{\beta_{2}}{\alpha_{2}}, 1\right)$

(ii) $m_{11} \in\left(\frac{\beta_{1}}{\alpha_{1}}, 1\right)$ and $m_{22} \in\left(0, \frac{\beta_{2}}{\alpha_{2}}\right]$

(iii) $m_{11} \in\left(\frac{\beta_{1}}{\alpha_{1}}, 1\right)$ and $m_{22} \in\left(\frac{\beta_{2}}{\alpha_{2}}, 1\right)$

(iv) $m_{11} \in\left(0, \frac{\beta_{1}}{\alpha_{1}}\right], m_{22} \in\left(0, \frac{\beta_{2}}{\alpha_{2}}\right]$ and $\left(\beta_{2}-\alpha_{2} m_{22}\right)\left(\beta_{1}-\alpha_{1} m_{11}\right)<\alpha_{1} \alpha_{2} m_{21} m_{12}$

When $\overline{\mathrm{x}}$ exists, it is unique. If, in addition, $\left(\beta_{2}-\alpha_{2} m_{22}\right)\left(\beta_{1}-\alpha_{1} m_{11}\right) \neq$ $\alpha_{1} \alpha_{2} m_{21} m_{12}$ and $\max \left\{m_{11}+m_{12}, m_{21}+m_{22}\right\}<1$, then $\overline{\mathrm{x}}$ is a global attractor on $\operatorname{int}\left(\mathbb{R}_{+}^{2}\right)$. 
Proof. The functions $f_{1}, f_{2}$ are concave by (41). Therefore, Theorem 5 can be applied. Since $\beta_{\ell}-\alpha_{\ell}<0$, then $\frac{\beta_{\ell}}{\alpha_{\ell}}<1$ and all intervals in (i) through (iii) are nontrivial. Condition (43) is equivalent to (A) of Theorem 5. Note that this expression is well defined in each of the cases (i) through (iv). Also, if $m_{11} \in\left(0, \frac{\beta_{1}}{\alpha_{1}}\right]$, then $m_{11} \frac{\alpha_{1}}{\beta_{1}} \leq 1$, which is equivalent to (B1). Similarly, $m_{22} \in\left(0, \frac{\beta_{2}}{\alpha_{2}}\right]$ is equivalent to $(\mathrm{C} 1)$.

If $m_{11} \in\left(\frac{\beta_{1}}{\alpha_{1}}, 1\right)$, then $m_{11} \alpha_{1}-\beta_{1}>0$ and $m_{11}<1$, in which case the equation $1=m_{11} f_{1}^{\prime}(t)$ has a positive solution given by

$$
t^{*}=\frac{\beta_{1}\left(1-m_{11}\right)-\sqrt{\beta_{1} m_{11}\left(\alpha_{1}-\beta_{1}\right)\left(1-m_{11}\right)}}{m_{11}-1} .
$$

Consequently, $m_{11} \in\left(\frac{\beta_{1}}{\alpha_{1}}, 1\right)$ implies that (B2) is satisfied. Similarly, $m_{22} \in\left(\frac{\beta_{2}}{\alpha_{2}}, 1\right)$ implies that $(\mathrm{C} 2)$ is satisfied. The existence and uniqueness of $\overline{\mathrm{x}}$ follows from Theorem 5. By Theorem 7, the origin is unstable and by Theorem 5, it is hyperbolic, hence $\rho\left(J_{T_{2}}(0)\right)>1$. Notice $M=\left(m_{i, j}\right)$ is irreducible and if we further assume that $\max \left\{m_{11}+m_{12}, m_{21}+m_{22}\right\}<1$, then $h_{+}(M)<1$. Consequently,

$$
\begin{aligned}
\overline{f^{\prime}(\infty)} & =\max _{\ell}\left\{\limsup _{t \rightarrow \infty} \frac{f_{\ell}(t)}{t}\right\} \\
& =\max _{\ell}\left\{\limsup _{t \rightarrow \infty} \frac{t+\alpha_{\ell}}{t+\beta_{\ell}}\right\}=1<\frac{1}{h_{+}(M)} .
\end{aligned}
$$

It follows from Theorem 3 that $\overline{\mathrm{x}}$ is a global attractor on $\operatorname{int}\left(\mathbb{R}_{+}^{2}\right)$.

It is worth mentioning that the global dynamics of (38) can be completely characterized using general theory of cooperative systems of difference equations. The example is presented only for illustrative purposes.

\subsection{Analysis of System (II)}

The map $T: \mathbb{R}_{+}^{k} \rightarrow \mathbb{R}_{+}^{k}$, given in (6), can be used to study more general structured population models. We specifically show that $T$ can be used to analyze systems of the form (II). 
Let $X$ be an ordered Banach space over $\mathbb{R}$, with solid order cone $X_{+}$. An element $\mathrm{x} \in X$ is said to be positive if $\mathrm{x} \in \operatorname{int}\left(X_{+}\right)$. Let $A$ be a nontrivial monotone bounded linear operator (so $A X_{+} \subset X_{+}$) such that the spectral radius of $A$ is less than one. Let $\mathrm{b}_{1}, \ldots, \mathrm{b}_{k}$ be $k \geq 1$ linearly independent elements of $X_{+}$, let $c_{1}, \ldots, c_{k}$ be positive bounded linear functionals on $X$, and let $f_{1}, \ldots, f_{k}$ be real valued functions as defined above. Consider the difference equation

$$
\mathrm{x}_{n+1}=A \mathrm{x}_{n}+\sum_{\ell=1}^{k} f_{\ell}\left(\mathrm{c}_{\ell} \mathrm{x}_{n}\right) \mathrm{b}_{\ell}, \quad \mathrm{x}_{0} \in X_{+} .
$$

Denote by $F$ the map corresponding to (II), i.e.,

$$
F(\mathrm{x})=A \mathrm{x}+\sum_{\ell=1}^{k} f_{\ell}\left(\mathrm{c}_{\ell} \mathrm{x}\right) \mathrm{b}_{\ell} .
$$

Thus $0 \in X$ is a fixed point of $F$. Let $C: X \rightarrow \mathbb{R}^{k}$ and $B: \mathbb{R}^{k} \rightarrow X_{+}$be the linear operators

$$
C(\mathrm{x}):=\left(\mathrm{c}_{1}(\mathrm{x}), \ldots, \mathrm{c}_{k}(\mathrm{x})\right), \quad \text { and } \quad B\left(s_{1}, \ldots, s_{k}\right):=\sum_{\ell=1}^{k} s_{\ell} \mathrm{b}_{\ell} .
$$

Define $M: \mathbb{R}^{k} \rightarrow \mathbb{R}^{k}$ from (6) as

$$
M:=C(I-A)^{-1} B
$$

and refer back to the map $T$ in (6), where now we have

$$
T(\mathrm{y})=M\left(f_{1}\left(\mathrm{y}^{(1)}\right), \ldots, f_{k}\left(\mathrm{y}^{(k)}\right)\right)^{t}=C(I-A)^{-1} B\left(f_{1}\left(\mathrm{y}^{(1)}\right), \ldots, f_{k}\left(\mathrm{y}^{(k)}\right)\right)^{t}
$$

We explore the relationship between fixed points of the map $T$ in (46) and the map $F$ in (44).

\subsubsection{Correspondence of Fixed Points}

The first result in this section establishes a correspondence between the fixed points of $T$ and the fixed points of $F$. 


\section{Theorem 8}

(i) If $\overline{\mathrm{x}} \in X_{+}$is a fixed point of $F$, then $\overline{\mathrm{t}} \in \mathbb{R}_{+}^{k}$ is a fixed point of $T$, where $\overline{\mathrm{t}}:=C(\overline{\mathrm{x}})$. If, additionally, $\overline{\mathrm{x}} \in \operatorname{int}\left(X_{+}\right)$, then $\overline{\mathrm{t}} \in \operatorname{int}\left(\mathbb{R}_{+}^{k}\right)$.

(ii) Let $\overline{\mathrm{x}}, \overline{\mathrm{y}} \in X_{+}$be distinct fixed points of $F$. Set $\overline{\mathrm{s}}=C(\overline{\mathrm{x}})$ and $\overline{\mathrm{t}}=C(\overline{\mathrm{y}})$. Then $\overline{\mathrm{s}} \neq \overline{\mathrm{t}}$.

(iii) If $\overline{\mathrm{t}} \in \mathbb{R}_{+}^{k}$ is a fixed point of $T$, then $\overline{\mathrm{x}}=\sum_{\ell=1}^{k} f_{\ell}\left(\overline{\mathrm{t}}_{\ell}\right)(I-A)^{-1} \mathrm{~b}_{\ell}$ is a fixed point of $F$ in $X_{+}$. Moreover, if $\overline{\mathrm{t}} \neq 0$ and $(I-A)^{-1} \mathrm{~b}_{\ell} \in \operatorname{int}\left(X_{+}\right)$for $\ell \in\{1, \ldots, k\}$, then $\overline{\mathrm{x}} \in \operatorname{int}\left(X_{+}\right)$.

(iv) Let $\overline{\mathrm{s}}$ and $\overline{\mathrm{t}}$ be distinct positive fixed points of $T$. Set $\overline{\mathrm{x}}:=\sum_{\ell=1}^{k} f_{\ell}\left(\overline{\mathrm{s}}_{\ell}\right)(I-$ $A)^{-1} \mathrm{~b}_{\ell}$ and $\overline{\mathrm{y}}:=\sum_{\ell=1}^{k} f_{\ell}\left(\overline{\mathrm{t}}_{\ell}\right)(I-A)^{-1} \mathrm{~b}_{\ell}$. If the functions $f_{1}, \ldots, f_{k}$ are one-to-one, then $\overline{\mathrm{x}} \neq \overline{\mathrm{y}}$.

Proof. (i) If $\overline{\mathrm{x}} \in X_{+}$is a positive fixed point of $F$, then $\overline{\mathrm{x}}=A \overline{\mathrm{x}}+\sum_{\ell=1}^{k} f_{\ell}\left(c_{\ell} \overline{\mathrm{x}}\right) \mathrm{b}_{\ell}$, and

$$
\overline{\mathrm{x}}=\sum_{\ell=1}^{k} f_{\ell}\left(c_{\ell} \overline{\mathrm{x}}\right)(I-A)^{-1} \mathrm{~b}_{\ell}
$$

Set $\overline{\mathrm{t}}_{\ell}:=c_{\ell}(\overline{\mathrm{x}})$ for $\ell \in\{1, \ldots, k\}$, and $\overline{\mathrm{t}}:=\left(\overline{\mathrm{t}}_{1}, \ldots, \overline{\mathrm{t}}_{k}\right)$. Then,

$$
\overline{\mathrm{t}}=C(\overline{\mathrm{x}})=\sum_{\ell=1}^{k} f_{\ell}\left(c_{\ell} \overline{\mathrm{x}}\right) C(I-A)^{-1} \mathrm{~b}_{\ell}=\sum_{\ell=1}^{k} f_{\ell}\left(\overline{\mathrm{t}}_{\ell}\right) C(I-A)^{-1} \mathrm{~b}_{\ell}=T(\overline{\mathrm{t}}) .
$$

If $\overline{\mathrm{x}} \in \operatorname{int}\left(X_{+}\right)$, then $\overline{\mathrm{t}}_{\ell}=c_{\ell}(\overline{\mathrm{x}})>0$ for $\ell \in\{1, \ldots, k\}$, so $\overline{\mathrm{t}} \in \operatorname{int}\left(\mathbb{R}_{+}^{k}\right)$.

(ii) If $\overline{\mathrm{x}}$ and $\overline{\mathrm{y}}$ are distinct positive fixed points of $F$ such that $C(\overline{\mathrm{x}})=C(\overline{\mathrm{y}})=$ $\left(t_{1}, \ldots, t_{k}\right)$, then

$$
\overline{\mathrm{x}}=A \overline{\mathrm{x}}+\sum_{\ell=1}^{k} f_{\ell}\left(t_{\ell}\right) \mathrm{b}_{\ell} \quad \text { and } \quad \overline{\mathrm{y}}=A \overline{\mathrm{y}}+\sum_{\ell=1}^{k} f_{\ell}\left(t_{\ell}\right) \mathrm{b}_{\ell} .
$$

Combine both equations in (47) to get

$$
\overline{\mathrm{x}}-\overline{\mathrm{y}}=A(\overline{\mathrm{x}}-\overline{\mathrm{y}}) .
$$


Since $\overline{\mathrm{x}} \neq \overline{\mathrm{y}}$, then (48) implies $\overline{\mathrm{x}}-\overline{\mathrm{y}}$ is an eigenvector of $A$ with corresponding eigenvalue 1 . This contradicts the hypothesis on the spectral radius of $A$. Thus $\overline{\mathrm{x}}=\overline{\mathrm{y}}$.

(iii) If $\overline{\mathrm{t}}$ is a fixed point of $T$ and $\overline{\mathrm{x}}=\sum_{\ell=1}^{k} f_{\ell}\left(\overline{\mathrm{t}}_{\ell}\right)(I-A)^{-1} \mathrm{~b}_{\ell}$, then

$$
C(\overline{\mathrm{x}})=\sum_{\ell=1}^{k} f_{\ell}\left(\overline{\mathrm{t}}_{\ell}\right) C(I-A)^{-1} \mathrm{~b}_{\ell}=T(\overline{\mathrm{t}})=\overline{\mathrm{t}} .
$$

Therefore $\overline{\mathrm{t}}_{\ell}=c_{\ell}(\overline{\mathrm{x}})$, for $\ell \in\{1, \ldots, k\}$. From this and the definition of $\overline{\mathrm{x}}$,

$$
(I-A) \overline{\mathrm{x}}=\sum_{\ell=1}^{k} f_{\ell}\left(\overline{\mathrm{t}}_{\ell}\right) \mathrm{b}_{\ell}=\sum_{\ell=1}^{k} f_{\ell}\left(c_{\ell}(\overline{\mathrm{x}})\right) \mathrm{b}_{\ell}
$$

whence $\overline{\mathrm{x}}$ is a fixed point of $F$. If in addition $\overline{\mathrm{t}} \neq 0$, then $\overline{\mathrm{x}} \neq 0$. By assumption $(I-A)^{-1} \mathrm{~b}_{\ell}>0$ for each $\ell \in\{1, \ldots, k\}$, hence solving for $\overline{\mathrm{x}}$ in (49) one can see that $\overline{\mathrm{x}}$ is a linear combination (with nonnegative coefficients) of elements of the interior of $X_{+}$, thus $\overline{\mathrm{t}} \neq 0$ implies $\overline{\mathrm{x}}>0$.

(iv) We have

$$
(I-A)(\overline{\mathrm{x}}-\overline{\mathrm{y}})=\sum_{\ell=1}^{k}\left(f_{\ell}\left(\overline{\mathrm{s}}_{\ell}\right)-f_{\ell}\left(\overline{\mathrm{t}}_{\ell}\right)\right) \mathrm{b}_{\ell}
$$

By assumption, for some $\ell_{0}$ we have $\overline{\mathrm{s}}_{\ell_{0}} \neq \overline{\mathrm{t}}_{\ell_{0}}$, and since $f_{\ell_{0}}$ is one-to-one, $f_{\ell_{0}}\left(\overline{\mathrm{s}}_{\ell_{0}}\right) \neq$ $f_{\ell_{0}}\left(\overline{\mathrm{t}}_{\ell_{0}}\right)$. By this relation and linear independence of the terms $\mathrm{b}_{\ell}, \sum_{\ell=1}^{k}\left(f_{\ell}\left(\overline{\mathrm{s}}_{\ell}\right)-\right.$ $\left.f_{\ell}\left(\overline{\mathrm{t}}_{\ell}\right)\right) \mathrm{b}_{\ell} \neq 0$. Thus the left-hand side of equation (50) is not zero. That is, $\mathrm{x} \neq \mathrm{y}$.

There is a corollary to Theorem 8 that is useful when it is known that the map $T$ has either no positive fixed points or a unique positive fixed point.

Corollary 5 If $T$ has no fixed points in int $\left(\mathbb{R}_{+}^{k}\right)$, then $F$ has no fixed points in $\operatorname{int}\left(X_{+}\right)$. If $T$ has a unique fixed point $\overline{\mathrm{t}} \in \operatorname{int}\left(\mathbb{R}_{+}^{k}\right)$, then $F$ has a unique fixed point $\overline{\mathrm{x}} \in \operatorname{int}\left(X_{+}\right)$, namely $\overline{\mathrm{x}}=\sum_{\ell=1}^{k} f_{\ell}\left(\overline{\mathrm{t}}_{\ell}\right)(I-A)^{-1} \mathrm{~b}_{\ell}$. 
Corollary 5 allows much of the theory developed in Sections 4.3 and 4.4 to be applied to systems of the form (II) by analyzing fixed points of the corresponding map $T$. These relationships are illustrated in the example provided in Section 4.5.4. First, we explore a deeper connection between the map $T$ in (46) and the map $F$ in (44), which involves the local stability character of the fixed point at the origin.

\subsubsection{A Linear Algebra Result}

We will see that in the case when $X=\mathbb{R}^{m}$ in (II), there is a strong connection between the stability of the origin in $X$ as a fixed point of $F$ and the stability of origin as a fixed point of $T$. To establish this connection, we shall prove a linear algebra result given in this subsection.

We will need the following lemma. We denote with $I_{m}$ the $m \times m$ identity matrix, $\operatorname{adj}(A)$ the adjugate of a square matrix $A$ (i.e., the transpose of the matrix of cofactors of $A), \rho(A)$ the spectral radius of $A$, and $\sigma(A)$ the spectrum of $A$.

Lemma 3 Let $A \in \mathbb{R}_{+}^{m \times m}$ be an irreducible matrix with eigenvalues $\lambda_{1}, \lambda_{2}, \ldots, \lambda_{m}$, where $\lambda_{1}$ denotes the spectral radius of $A$. Then (i) the matrix $\operatorname{adj}\left(\lambda_{1} I_{m}-A\right)$ has nonnegative entries, and (ii) $\operatorname{tr}\left[\operatorname{adj}\left(\lambda_{1} I_{m}-A\right)\right]=\left(\lambda_{1}-\lambda_{2}\right) \cdots\left(\lambda_{1}-\lambda_{m}\right)$.

Proof. For $t>\lambda_{1}$ the matrix $\operatorname{adj}\left(t I_{m}-A\right)$ is invertible, and,

$$
\operatorname{adj}\left(t I_{m}-A\right)=\operatorname{det}\left(t I_{m}-A\right)\left(t I_{m}-A\right)^{-1}
$$

Note $\operatorname{det}\left(t I_{m}-A\right)=\left(t-\lambda_{1}\right) \cdots\left(t-\lambda_{m}\right)>0$. Also, the Neumann series expansion $\left(I_{m}-\frac{1}{t} A\right)^{-1}=I+\frac{1}{t} A+\frac{1}{t^{2}} A^{2}+\cdots$ and $A$ irreducible imply that $\left(t I_{m}-A\right)^{-1}$ has positive entries.. Since $\operatorname{adj}\left(\lambda_{1} I_{m}-A\right)=\lim _{t \downarrow \lambda_{1}} \operatorname{adj}\left(t I_{m}-A\right)$, it follows that $\operatorname{adj}\left(\lambda_{1} I_{m}-A\right)$ has nonnegative entries. For the proof of statement (ii), the relation

$$
\operatorname{tr}(\operatorname{adj}(t I-A))=\operatorname{det}(t I-A) \operatorname{tr}\left((t I-A)^{-1}\right)=\prod_{\ell=1}^{m}\left(t-\lambda_{\ell}\right) \sum_{\ell=1}^{m}\left(t-\lambda_{\ell}\right)^{-1}
$$


implies

$$
\operatorname{tr}(\operatorname{adj}(t I-A))=\sum_{\ell=1}^{m} \prod_{j \neq \ell}\left(t-\lambda_{\ell}\right) .
$$

Statement (ii) follows by taking $t=\lambda_{1}$ in (53).

Proposition 4 Let $A \in \mathbb{R}_{+}^{m \times m}, B \in \mathbb{R}_{+}^{m \times k}$ and $C \in \mathbb{R}_{+}^{k \times m}$. Suppose $A$ is irreducible, $\rho(A)<1$ and $\rho(B C) \neq 0$. Set $E:=\left(I_{m}-A\right)^{-1} B$. Then,

(i) $1 \in \sigma(A+B C)$ if and only if $1 \in \sigma(C E)$

(ii) Let $\bar{\rho}_{1}:=\rho(A+B C)$ and $\bar{\rho}_{2}:=\rho(C E)$. Then $\operatorname{sgn}\left(\bar{\rho}_{1}-1\right)=\operatorname{sgn}\left(\bar{\rho}_{2}-1\right)$

Proof. From Sylvester's determinant identity [16] we have,

$\operatorname{det}\left(\left(I_{m}-A\right)^{-1}\right) \operatorname{det}\left(I_{m}-A-B C\right)=\operatorname{det}\left(I_{m}-\left(I_{m}-A\right)^{-1} B C\right)=\operatorname{det}\left(I_{k}-C\left(I_{m}-A\right)^{-1} B\right)$, from which statement (i) follows.

For $t \geq 0$, let $\rho_{1}(t)$ denote the spectral radius of $A+t B C$, and let $\rho_{2}(t)$ denote the spectral radius of $t C E$. We claim that $\rho_{\ell}(t)$ is a monotonically increasing function of $t$ for $t \geq 0$. Since for each $t, \rho_{\ell}(t)$ is a simple root of the characteristic polynomial, by the Implicit Function Theorem $\rho_{\ell}(\cdot)$ is a $C^{(1)}$ function of $t$. Define the functions

$$
\phi_{1}(t):=\operatorname{det}\left(\rho_{1}(t) I_{m}-A-t B C\right) \quad \text { and } \quad \phi_{2}(t):=\operatorname{det}\left(\rho_{2}(t) I_{k}-t C E\right) \text {. }
$$

Hence

$$
\phi_{1}(t)=0 \quad \text { and } \quad \phi_{2}(t)=0 \text { for } t \geq 0 \text {. }
$$

Jacobi's formula for the derivative of the determinant of a matrix function of a real variable and the relations $\phi_{1}^{\prime}(t)=0$ and $\phi_{2}^{\prime}(t)=0$ give

$$
\rho_{1}^{\prime}(t)=\frac{\operatorname{tr}\left(\operatorname{adj}\left(\rho_{1}(t) I_{m}-A-t B C\right) B C\right)}{\operatorname{tr}\left(\operatorname{adj}\left(\rho_{1}(t) I_{m}-A-t B C\right)\right)}
$$




$$
\rho_{2}^{\prime}(t)=\frac{\operatorname{tr}\left(\operatorname{adj}\left(\rho_{2}(t) I_{k}-t C E\right) C E\right)}{\operatorname{tr}\left(\operatorname{adj}\left(\rho_{2}(t) I_{k}-t C E\right)\right)}
$$

Note in relations (55), (56) the denominator is positive by (ii) of Lemma 3, and the numerator is nonnegative by (i) of Lemma 3 , that is, $\rho_{1}(t) \geq 0$ and $\rho_{2}(t) \geq$ 0 for $t \geq 0$. Again by (i) of Lemma 3, at least one diagonal entry of each of $\operatorname{adj}\left(\rho_{1}(t) I_{m}-A-t B C\right)$ and $\operatorname{adj}\left(\rho_{2}(t) I_{k}-t C E\right)$ is positive. Given that the matrices $B C$ and $C E$ have nonnegative entries and have at least one positive diagonal entry (since $\rho(B C)>0$ by hypothesis), it follows that the numerators in $(55),(56)$ are necessarily positive, that is, the functions $\rho_{1}(\cdot)$ and $\rho_{2}(\cdot)$ are monotonically increasing.

We now verify that $\rho_{1}(t)$ takes values smaller than one and also larger than one. Note that $\rho_{1}(0)=\rho(A)<1$. Choose $t^{\prime}>\rho(B C)^{-1}$. By Corollary 8.1.19 in [19], $\rho\left(A+t^{\prime} B C\right) \geq \rho\left(t^{\prime} B C\right)=t^{\prime} \rho(B C)>1$, that is, $\rho_{1}\left(t^{\prime}\right) \geq 1$.

Since $\rho_{1}(t)$ takes values less than, greater than, and (by continuity) equal to one, by statement (i) and monotonicity of $\rho_{\ell}, \ell=1,2$, it follows that $\rho_{1}(t)>1$ if and only if $\rho_{2}(t)>1, \rho_{1}(t)<1$ if and only if $\rho_{2}(t)<1$, and $\rho_{1}(t)=1$ if and only if $\rho_{2}(t)=1$. Statement (ii) of the proposition now follows from the latter relations and the equalities $\bar{\rho}_{1}=\rho_{1}(1)$ and $\bar{\rho}_{2}=\rho_{2}(1)$.

Proposition 4 can now be utilized to discuss the relationship between the stability character of the origin as a fixed point of the map $T$ and as a fixed point of the map $F$.

\subsubsection{Stability of the Origin}

We now take $X=\mathbb{R}^{m}$ with the standard order cone $\mathbb{R}_{+}^{m}=\left\{\left(x_{1}, \ldots, x_{m}\right)\right.$ : $\left.x_{\ell} \geq 0, \ell=1, \ldots m\right\}$. Let $A \in \mathbb{R}_{+}^{m \times m}, B \in \mathbb{R}_{+}^{m \times k}$ and $C \in \mathbb{R}_{+}^{k \times m}$. For $\ell=1, \ldots, k$, denote with $c_{\ell}$ the $\ell$-th row of $C$, and define $c_{\ell} \mathrm{x}$ to be the inner product of $c_{\ell}$ and $\mathrm{x}$, that is $c_{\ell} \mathrm{x}=\mathrm{c}_{\ell} \cdot \mathrm{x}$. Suppose $\rho(A)<1$, and that $B$ has full column rank. 
The next result states that for system (I), the local stability character of $0 \in \mathbb{R}^{m}$ as a fixed point of $F$ is the same as the local stability character of $0 \in \mathbb{R}^{k}$ as a fixed point of $T$ in (46). By $J_{F}(\mathrm{x})$ and $J_{T}(\mathrm{t})$ we denote the Jacobian matrices of $F$ and $T$ at $\mathrm{x}$ and $\mathrm{t}$ respectively.

Theorem 9 If $A$ is irreducible and $\rho\left(B \operatorname{diag}\left(f_{1}^{\prime}(0), \ldots, f_{k}^{\prime}(0)\right) C\right) \neq 0$, then the following statements are true.

(i) $\rho\left(J_{T}(0)\right)=1$ if and only if $\rho\left(J_{F}(0)\right)=1$.

(ii) $\rho\left(J_{T}(0)\right)<1$ if and only if $\rho\left(J_{F}(0)\right)<1$.

(iii) $\rho\left(J_{T}(0)\right)>1$ if and only if $\rho\left(J_{F}(0)\right)>1$.

Proof. Set $D_{0}:=\operatorname{diag}\left(f_{1}^{\prime}(0), \ldots, f_{k}^{\prime}(0)\right)$ and $E:=(I-A)^{-1} B D_{0}$. The Jacobian matrix of the map $T$ at $0 \in \mathbb{R}^{k}$ is

$$
J_{T}(0)=C(I-A)^{-1} B D_{0}=C E
$$

The Jacobian matrix of $F$ at $0 \in \mathbb{R}^{m}$ is

$$
J_{F}(0)=A+\sum_{j=1}^{k} f_{j}^{\prime}(0) \mathrm{b}_{j} \mathrm{c}_{j} \cdot=A+B D_{0} C
$$

Set $\tilde{B}=B D_{0}$ in (58) and (57). In this case, we have

$$
J_{T}(0)=C(I-A)^{-1} \tilde{B} \quad \text { and } \quad J_{F}(0)=A+\tilde{B} C
$$

Now, comparison of 1 and the spectral radii $\rho(A+\tilde{B} C)$ and $\rho(C E)$ is obtained from Proposition 4, which gives the conclusion of the Theorem.

Combining the results from Section 4.3, Corollary 5, and Theorem 9, global results can be established for systems of the form (II). A relevant question in this regard is the following: Is it the case that when the map T from (46) has a globally 
asymptotically stable positive fixed point, then the map F from (44) has a globally asymptotically stable positive fixed point? This question will be explored in future work.

\subsubsection{Examples}

To illustrate the connection between all of the results of the preceeding sections, we consider an example of a system with the form (44) for $k=2$, where the functions are taken to be of Beverton-Holt type (4). This example requires results established in Example 1.

Example 3: Consider the system of difference equations

$$
\mathrm{x}_{n+1}=A \mathrm{x}_{n}+\frac{\mathrm{c}_{1} \mathrm{x}_{n}}{\mathrm{c}_{1} \mathrm{x}_{n}+\delta_{1}} \mathrm{~b}_{1}+\frac{\mathrm{c}_{2} \mathrm{x}_{n}}{\mathrm{c}_{2} \mathrm{x}_{n}+\delta_{2}} \mathrm{~b}_{2}
$$

where $\mathrm{x}_{n}, \mathrm{x}_{0}, \mathrm{~b}_{1}, \mathrm{~b}_{2} \in \mathbb{R}_{+}^{m}, A \in \mathbb{R}_{+}^{m} \times \mathbb{R}_{+}^{m}, \delta_{1}, \delta_{2}>0$, and $\mathrm{c}_{1}, \mathrm{c}_{2}$ are positive row vectors in $\mathbb{R}_{+}^{m}$. This system has the form given in (44) where $f_{\ell}(t)=\frac{t}{t+\delta_{\ell}}$ is of Beverton-Holt type. Denote by $F_{3}: \mathbb{R}_{+}^{m} \rightarrow \mathbb{R}_{+}^{m}$ the map corresponding to (59). That is,

$$
F_{3}(\mathrm{y})=A \mathrm{y}+\frac{\mathrm{c}_{1} \mathrm{y}}{\mathrm{c}_{1} \mathrm{y}+\delta_{1}} \mathrm{~b}_{1}+\frac{\mathrm{c}_{2} \mathrm{y}}{\mathrm{c}_{2} \mathrm{y}+\delta_{2}} \mathrm{~b}_{2}
$$

Define $\mathrm{v}_{\ell}=(I-A)^{-1} \mathrm{~b}_{\ell}$ for $\ell=1,2$ and let $M \in \mathbb{R}_{+}^{2} \times \mathbb{R}_{+}^{2}$ be as in (45). That is,

$$
M=\left(\begin{array}{ll}
\mathrm{c}_{1} \mathrm{v}_{1} & \mathrm{c}_{1} \mathrm{v}_{2} \\
\mathrm{c}_{2} \mathrm{v}_{1} & \mathrm{c}_{2} \mathrm{v}_{2}
\end{array}\right)
$$

Consider the map $T_{3}: \mathbb{R}_{+}^{2} \rightarrow \mathbb{R}_{+}^{2}$ defined in (46), given by

$$
\begin{aligned}
T_{3}\left(\begin{array}{l}
u \\
v
\end{array}\right) & =\left(\begin{array}{l}
\mathrm{c}_{1} \mathrm{v}_{1} f_{1}(u)+\mathrm{c}_{1} \mathrm{v}_{2} f_{2}(v) \\
c_{2} \mathrm{v}_{1} f_{1}(u)+\mathrm{c}_{2} \mathrm{v}_{2} f_{2}(v)
\end{array}\right) \\
& =\left(\begin{array}{l}
\frac{\mathrm{c}_{1} \mathrm{v}_{1} u}{\delta_{1}+u}+\frac{\mathrm{c}_{1} \mathrm{v}_{2} v}{\delta_{2}+v} \\
\frac{\mathrm{c}_{2} \mathrm{v}_{1} u}{\delta_{1}+u}+\frac{\mathrm{c}_{2} \mathrm{v}_{2} v}{\delta_{2}+v}
\end{array}\right),
\end{aligned}
$$

Compare (61) to the map studied in Example 1, where $m_{i, j}=\mathrm{c}_{i} \mathrm{v}_{j}$. There exists a correspondence between the fixed points of $T_{3}$ and the fixed points of $F_{3}$ as discussed in the previous section. This leads to the following claim: 
Claim 1 Suppose that

$$
\left(\delta_{2}-\mathrm{c}_{2} \mathrm{v}_{2}\right)\left(\delta_{1}-\mathrm{c}_{1} \mathrm{v}_{1}\right)<\mathrm{c}_{1} \mathrm{v}_{2} \mathrm{c}_{2} \mathrm{v}_{1}
$$

The map $F_{3}$ has a positive fixed point $\overline{\mathrm{y}}$ if and only if at least one of the following conditions is satisfied:

(i) $\mathrm{c}_{1} \mathrm{v}_{1} \leq \delta_{1}$ and $\mathrm{c}_{2} \mathrm{v}_{2}>\delta_{2}$ or $\mathrm{c}_{1} \mathrm{v}_{1}>\delta_{1}$ and $\mathrm{c}_{2} \mathrm{v}_{2} \leq \delta_{2}$.

(ii) $\mathrm{c}_{1} \mathrm{v}_{1}>\delta_{1}$ and $\mathrm{c}_{2} \mathrm{v}_{2}>\delta_{2}$.

(iii) $\mathrm{c}_{1} \mathrm{v}_{1} \leq \delta_{1}, \mathrm{c}_{2} \mathrm{v}_{2} \leq \delta_{2}$ and $\left(\delta_{2}-\mathrm{c}_{2} \mathrm{v}_{2}\right)\left(\delta_{1}-\mathrm{c}_{1} \mathrm{v}_{1}\right)<\mathrm{c}_{1} \mathrm{v}_{2} \mathrm{c}_{2} \mathrm{v}_{1}$.

Furthermore, if it exists, $\overline{\mathrm{y}}$ is unique and locally asymptotically stable.

Proof. Under any of the conditions (i) through (iii), it follows from Proposition 1 that the map $T_{3}$ from (61) has a unique positive fixed point $\overline{\mathrm{x}}$. The existence and uniqueness of a positive fixed point $\bar{y}$ for the map $F_{3}$ then follows from Corollary 5 .

Now, the local stability character of $0 \in \mathbb{R}_{+}^{2}$ as a fixed point of $T_{3}$ is the same as the local stability character of $0 \in \mathbb{R}_{+}^{m}$ as a fixed point of $F_{3}$ by Theorem 9 . By Theorem 7, $0 \in \mathbb{R}^{2}$ is an unstable, hyperbolic fixed point of $T_{3}$ and thus $0 \in \mathbb{R}^{m}$ is an unstable, hyperbolic fixed point of $F_{3}$. By results of monotone systems, and an argument similar to the one used in the proof of Theorem 7, this implies that the unique positive fixed point of the map $F_{3}$ is locally asymptotically stable.

\section{Numerical Examples}

Corresponding to Examples 1 and 2 from Section 4.4.3, we present two numerical examples. For Example 1, consider system (33), where $m_{11}=4, m_{12}=3$, 

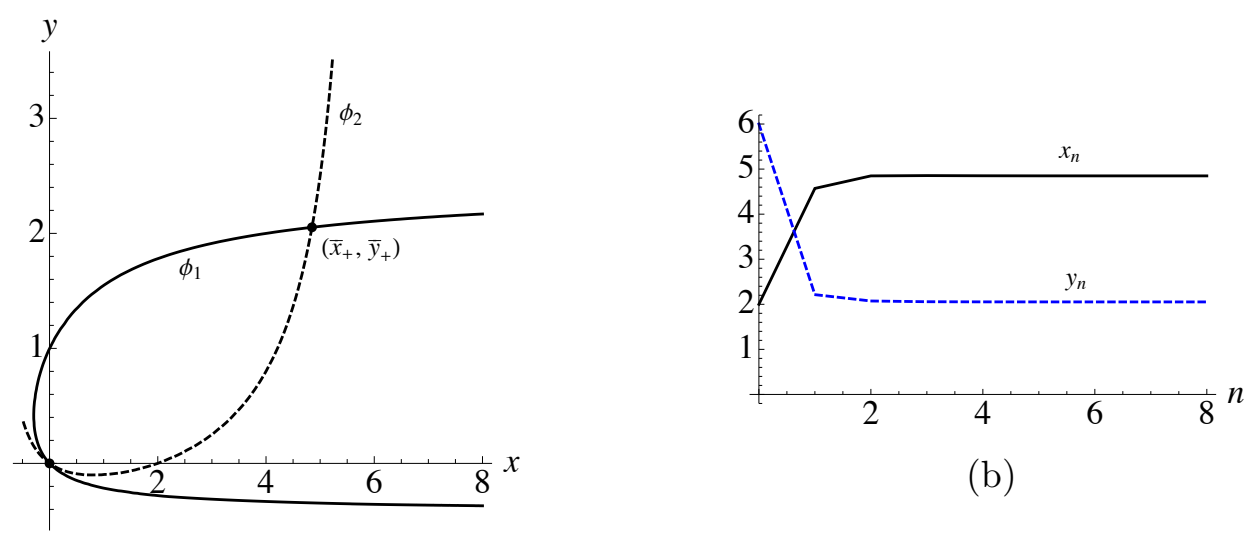

(b)

(a)

Figure 19: The curves $\phi_{1}$ and $\phi_{2}$ corresponding to system (62) can be seen in (a). A solution $\left(x_{n}, y_{n}\right)$ to $(62)$ with $\left(x_{0}, y_{0}\right)=(2,6)$ can be seen in (b). The solution quickly approaches the equilibrium point $\overline{\mathrm{x}}$ given in (63).

$m_{21}=1, m_{22}=2, \delta_{1}=2$, and $\delta_{2}=1$. That is, consider

$$
\begin{aligned}
& x_{n+1}=\frac{4 x_{n}}{2+x_{n}}+\frac{3 y_{n}}{1+y_{n}}, \\
& y_{n+1}=\frac{x_{n}}{2+x_{n}}+\frac{2 y_{n}}{1+y_{n}}, \quad n=0,1,2, \ldots,
\end{aligned}
$$

where $\left(x_{0}, y_{0}\right) \in(0, \infty) \times(0, \infty)$. It can be easily verified that the conditions set forth in Proposition 1 are satisfied. It follows that system (62) has a unique positive equilibrium point. This equilibrium is given by

$$
\overline{\mathrm{x}} \approx(4.849,2.053)
$$

where the coordinates have been rounded to three decimal places. The equilibrium $\overline{\mathrm{x}}$ is a global attractor on $\operatorname{int}\left(\mathbb{R}_{+}^{2}\right)$ by Proposition 1 . The equilibrium point, along with the parametric curves $\phi_{1}$ and $\phi_{2}$ for this case (defined in (15)), can be seen in (a) of Figure 19. A solution to $(62)$ for $\left(x_{0}, y_{0}\right)=(2,6)$ can be seen in (b) of Figure 19.

For Example 2, Consider system (38), where $m_{11}=\frac{1}{2}, m_{12}=\frac{1}{2}, m_{21}=1$, 
$m_{22}=2, \alpha_{1}=2, \alpha_{2}=1$ and $\beta_{1}=\beta_{2}=4$. That is, consider the system

$$
\begin{aligned}
& x_{n+1}=\frac{x_{n}\left(x_{n}+2\right)}{2\left(x_{n}+4\right)}+\frac{y_{n}\left(y_{n}+1\right)}{2\left(y_{n}+4\right)}, \\
& y_{n+1}=\frac{x_{n}\left(x_{n}+2\right)}{x_{n}+4}+\frac{2 y_{n}\left(y_{n}+1\right)}{y_{n}+4}, \quad n=0,1,2, \ldots
\end{aligned}
$$

It can be verified that system (64) satisfies the conditions of Proposition 2 and therefore has a unique positive equilibrium point. The positive equilibrium is given by

$$
\overline{\mathrm{x}}=\left(\frac{\sqrt{969}-27}{12}, \frac{3 \sqrt{969}-41}{44}\right) \approx(0.344,1.191)
$$

Theorem 7 guarantees that the fixed point at the origin is locally asymptotically stable and Theorem 6 allows us to conclude that $\overline{\mathrm{x}}$ is unstable. In fact, the eigenvalues of the Jacobian evaluated at $\overline{\mathrm{x}}$ are

$$
\lambda_{1} \approx 1.272 \text { and } \lambda_{2} \approx 0.126
$$

and thus $\overline{\mathrm{x}}$ is a saddle point. It can also be verified that there do not exist any minimal period two solutions in $\mathbb{R}_{+}^{2}$. Therefore, applying results from $[20,21,22]$, there exists the global stable manifold $W^{s}(\overline{\mathrm{x}})$ and the global unstable manifold $W^{u}(\overline{\mathrm{x}})$ passing through $\overline{\mathrm{x}}$ and extending to the boundary of the first quadrant (depicted in (b) of Figure 20). The region in the first quadrant below (resp. above) the curve $W^{s}(\overline{\mathrm{x}})$ is the basin of attraction of the origin (resp. the point $(+\infty,+\infty))$ and the curve $W^{s}(\overline{\mathrm{x}})$ is precisely the basin of attraction of $E$.

The equilibrium point, along with the parametric curves $\phi_{1}$ and $\phi_{2}$ for this case (defined in (15)), can be seen in (a) of Figure 20. A solution of (64) with an initial condition chosen from below the global stable manifold can be seen in (a) of Figure 21 and a solution of (64) with an initial condition chosen from above the global stable manifold be seen in (b) of Figure 21. 


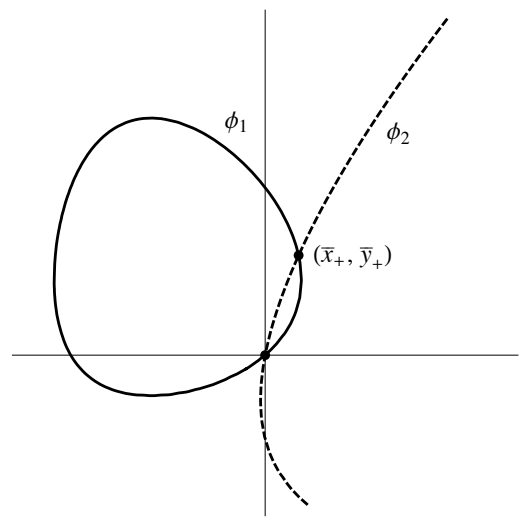

(a)

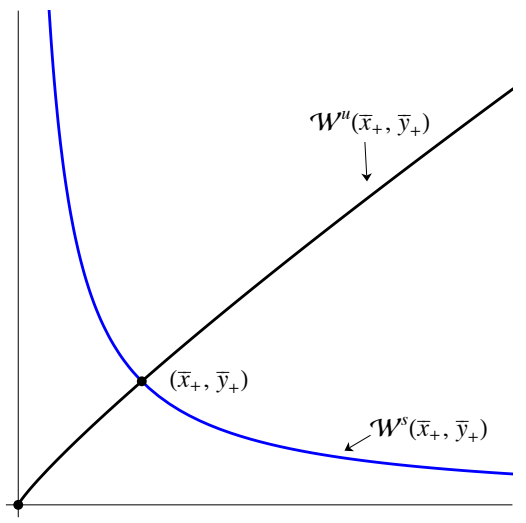

(b)

Figure 20: The curves $\phi_{1}$ and $\phi_{2}$ corresponding to system (64) can be seen in (a). The global stable and unstable manifolds, $\mathcal{W}^{s}$ and $\mathcal{W}^{u}$, corresponding to the interior saddle point $\overline{\mathrm{x}}$ can be seen in (b).

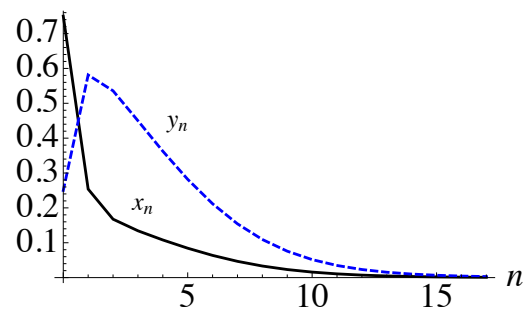

(a)

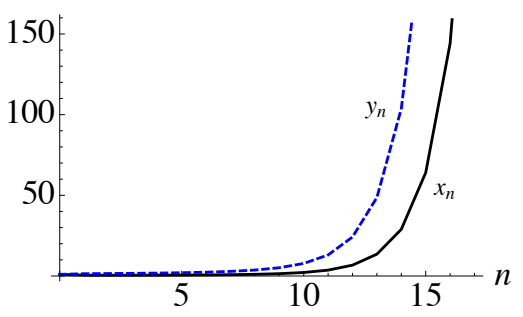

(b)

Figure 21: A solution $\left(x_{n}, y_{n}\right)$ to $(64)$ with $\left(x_{0}, y_{0}\right)=\left(\frac{3}{4}, \frac{1}{4}\right)$ can be seen in (a). The solution quickly converges to the origin. A solution $\left(x_{n}, y_{n}\right)$ to (64) with $\left(x_{0}, y_{0}\right)=(1,1)$ can be seen in (b). The solution approaches $(+\infty,+\infty)$. 


\section{List of References}

[1] J. M. Cushing, An Introduction to Structured Population Dynamics. Philadelphia: SIAM, 1998.

[2] F. Brauer and C. Castillo-Chávez, Mathematical Models in Population Biology and Epidemiology. New York: Springer-Verlag, 2001.

[3] A. Ackleh, B. Ma, and R. Miller, "A general nonlinear model for the interaction of a size-structured population and its environment," Quarterly of Applied Mathematics, vol. 74, pp. 671-704, 2016.

[4] N. Grosjean and T. Huillet, "On simple age-structured population models," Applied Mathematical Modeling, vol. 41, pp. 68-82, 2017.

[5] M. Jones, "A discrete stage-structured model of california newt population dynamics during a period of drought," Journal of Theoretical Biology, vol. 414, pp. 245-253, 2017.

[6] J. Li, L. Cali, and Y. Li, "Stage-structured wild and sterile mosquito population models and their dynamics," Journal of Biological Dynamics, vol. 11, pp. 79-101, 2017.

[7] S. Friedland and S. Karlin, "Some inequalities for the spectral radius of nonnegative matrices and applications," Duke Mathematical Journal, vol. 42, pp. 459-489, 1975.

[8] R. Rebarber, B. Tenhumberg, and S. Townley, "Global asymptotic stability of density dependent integral projection models," Theoretical Population Biology, vol. 81, pp. 81-87, 2012.

[9] S. Townley, R. Rebarber, and B. Tenhumberg, "Feedback control systems analysis of density dependent population dynamics," Systems and Control Letters, vol. 61, pp. 309-315, 2012.

[10] E. Pardini, J. Drake, J. Chase, and T. Knight, "Complex population dynamics and control of the invasive biennial alliaria petiolata," Ecological Applications, vol. 19, pp. 387-397, 2009.

[11] M. Rees and K. Rose, "Evolution of flowering strategies in oenothera glazioviana: an integral projection model approach," Proceedings of the Royal Society of London, vol. 269, pp. 1509-1515, 2002.

[12] H. L. Smith and H. R. Thieme, "Persistence and global stability for a class of discrete time structured population models," Discrete and Continuous Dynamical Systems, vol. 33, pp. 4627-4646, 2013. 
[13] K. Deimling, Nonlinear Functional Analysis. New York: Springer Verlag, 1985.

[14] M. Hirsch and H. Smith, Handbook of Differential Equations: Ordinary Differential Equations; Monoton Dynamical Systems. Amsterdam: Elsevier B. V., 2005.

[15] S. Sternberg, Dynamical Systems. Dover Publications, 2013.

[16] A. G. Akritas, E. K. Akritas, and G. I. Malaschonok, "Various proofs of sylvester's (determinant) identity," Mathematics and Computers in Simulation, vol. 42, pp. 585-593, 1996.

[17] S. Basu and O. Merino, "On the behavior of solutions of a system of difference equations," Comm. Appl. Nonlinear Anal., vol. 16, pp. 89-101, 2009.

[18] E. N. Dancer and P. Hess, "Stability of fixed points for order-preserving discrete-time dynamical systems," J. reine angew. Math., vol. 419, pp. 125139, 1991.

[19] R. A. Horn and C. R. Johnson, Matrix Analysis. New York: Cambridge University Press, 1985.

[20] M. R. S. Kulenović and O. Merino, "Competitive-exclusion versus competitive-coexistence for systems in the plane," Discrete Contin. Dyn. Syst. Ser. B, vol. 6, pp. 1141-1156, 2006.

[21] M. R. S. Kulenović and O. Merino, "Global bifurcation for competitive systems in the plane," Discrete Contin. Dyn. Syst. Ser. B, vol. 12, pp. 133-149, 2009.

[22] M. R. S. Kulenović and O. Merino, "Invariant manifolds for competitive discrete systems in the plane," Int. J. of Bifurcations and Chaos, vol. 20, pp. 2471-2486, 2010. 


\section{BIBLIOGRAPHY}

Ackleh, A., Ma, B., and Miller, R., "A general nonlinear model for the interaction of a size-structured population and its environment," Quarterly of Applied Mathematics, vol. 74, pp. 671-704, 2016.

Akritas, A. G., Akritas, E. K., and Malaschonok, G. I., "Various proofs of sylvester's (determinant) identity," Mathematics and Computers in Simulation, vol. 42, pp. 585-593, 1996.

Allen, L. J. S., An Introduction to Mathematical Biology. Upper Saddle River, New Jersey: Pearson Prentice Hall, 1987.

Andrews, J. G. and McLone, R. R., Mathematical Modeling. London: Butterworth-Heinwmann, 1976.

Basu, S. and Merino, O., "On the behavior of solutions of a system of difference equations," Comm. Appl. Nonlinear Anal., vol. 16, pp. 89-101, 2009.

Bilgin, A. and Kulenović, M. R. S., "Two species competitive model with the allee effect," Advances in Difference Equations, vol. 307, pp. 1-27, 2014.

Bilgin, A., Kulenović, M. R. S., and Pilav, E., "Basins of attraction of period-two solutions of monotone systems of difference equations," Advances in Difference Equations, vol. 74, pp. 1-25, 2016.

Brauer, F. and Castillo-Chávez, C., Mathematical Models in Population Biology and Epidemiology. New York: Springer-Verlag, 2001.

Burgić, D., Kalabušić, S., and Kulenović, M. R. S., "Non-hyperbolic dynamics for competitive systems in the plane and global period-doubling bifurcations," Adv. Dyn. Syst. Appl., vol. 3, pp. 229-249, 2008.

Cai, Y., Wang, Z., and Wang, W., "Endemic dynamics in a host-parasite epidemiological model within spatially heterogeneous environment," Applied Mathematics Letters, vol. 61, pp. 129-136, 2016.

Camouzis, E., Kulenović, M. R. S., Ladas, G., and Merino, O., "Rational systems in the plane - open problems and conjectures," Journal of Difference Equations and Applications, vol. 15, pp. 303-323, 2009.

Clark, D., Kulenović, M. R. S., and Selgrade, J. F., "Global asymptotic behavior of a two dimensional difference equation modelling competition," Nonlinear Anal., vol. 52, pp. 1765-1776, 2003. 
Cushing, J. M., An Introduction to Structured Population Dynamics. Philadelphia: SIAM, 1998.

Cushing, J., Levarge, S., Chitnis, N., , and Henson, S., "Some discrete competition models and the competitive exclusion principle," Journal of Difference Equations and Applications, vol. 10, pp. 1139-1151, 2004.

Dancer, E. N. and Hess, P., "Stability of fixed points for order-preserving discretetime dynamical systems," J. reine angew. Math., vol. 419, pp. 125-139, 1991.

Deimling, K., Nonlinear Functional Analysis. New York: Springer Verlag, 1985.

Din, Q., "Global behavior of a host-parasitoid model under the constant refuge effect," Applied Mathematical Modeling, vol. 40, pp. 2815-2826, 2016.

Din, Q. and Donchev, T., "Global character of a host-parasite model," Chaos, Solitons \&f Fractals, vol. 54, pp. 1-7, 2013.

Elaydi, S., An Introduction to Difference Equations, 3rd Ed. New York: SpringerVerlage, 2005.

Ellner, S. and Guckenheimer, J., Dynamic Models in Biology. Princeton, New Jersey: Princeton University Press, 2006.

Friedland, S. and Karlin, S., "Some inequalities for the spectral radius of nonnegative matrices and applications," Duke Mathematical Journal, vol. 42, pp. 459-489, 1975.

Gidea, M., Meiss, J. D., Ugarcovici, I., and Weiss, H., "Applications of kam thoery to population dynamics," Journal of Biological Dynamics, vol. 1, pp. 1-23, 2009.

Gómez-Corral, A. and García, M. L., "Control strategies for a stochastic model of host-parasite interaction in a seasonal environment," Journal of Theoretical Biology, vol. 354, pp. 1-11, 2014.

Grosjean, N. and Huillet, T., "On simple age-structured population models," $A p$ plied Mathematical Modeling, vol. 41, pp. 68-82, 2017.

Hassell, M., The Spatial and Temporal Dynamics of Host-Parasitoid Interactions. New York: Oxford University Press, 2000.

Hawkins, B., Pattern and Process in Host-Parasitoid Interactions. New York: Cambridge University Press, 1994.

Hess, P., Periodic-parabolic boundary value problems and positivity. New York: Longman Scientific \& Technical, Harlow, 1991. 
Hirsch, M. and Smith, H., Handbook of Differential Equations: Ordinary Differential Equations; Monoton Dynamical Systems. Amsterdam: Elsevier B. V., 2005.

Horn, R. A. and Johnson, C. R., Matrix Analysis. New York: Cambridge University Press, 1985.

Huang, Y. S. and Knopf, P. M., "Global convergence properties of first-order homogeneous systems of rational difference equations," Journal of Difference Equations and Applications, vol. 18, pp. 1683-1707, 2012.

Jamieson, W. T. and Merino, O. University of Rhode Island. "Local dynamics of planar maps with a non-isolated fixed point exhibiting 1-1 resonance." 2017. [Online]. Available: http://math.uri.edu/ merino/JamiesonMerino2017.pdf

Jones, M., "A discrete stage-structured model of california newt population dynamics during a period of drought," Journal of Theoretical Biology, vol. 414, pp. 245-253, 2017.

Kalabušić, S., Kulenović, M. R. S., and Pilav, E., "Dynamics of a two-dimensional system of rational difference equations of leslie-gower type," Advances in Difference Equations, vol. 29, pp. 1-29, 2011.

Kang, Y., Sasmal, S., Bhowmick, A., and Chattopadhyay, J., "A host-parasitoid system with predation-driven component allee effects in host population," Journal of Biological Dynamics, vol. 9, pp. 213-232, 2015.

Khan, A. and Qureshi, M., "Dynamics of a modified nicholson-bailey hostparasitoid model," Advances in Difference Equations, vol. 23, pp. 1-15, 2015.

Krause, U., Positive Dynamical Systems in Discrete Time. Berlin: De Gruyter, 2015.

Kulenović, M. R. S. and Merino, O., Discrete Dynamical Systems and Difference Equations with Mathematica. Boca Raton: Chapman\& Hall/CRC Press, 2002.

Kulenović, M. R. S. and Merino, O., "Competitive-exclusion versus competitivecoexistence for systems in the plane," Discrete Contin. Dyn. Syst. Ser. B, vol. 6, pp. 1141-1156, 2006.

Kulenović, M. R. S. and Merino, O., "A global attractivity result for maps with invariant boxes," Discrete and Continuous Dynamical Systems, vol. 6, pp. 97-110, 2006.

Kulenović, M. R. S. and Merino, O., "Global bifurcation for competitive systems in the plane," Discrete Contin. Dyn. Syst. Ser. B, vol. 12, pp. 133-149, 2009. 
Kulenović, M. R. S. and Merino, O., "Invariant manifolds for competitive discrete systems in the plane," Int. J. of Bifurcations and Chaos, vol. 20, pp. 24712486, 2010.

Kulenović, M. R. S. and Nurkanović, M., "Asymptotic behavior of a twodimensional linear fractional system of difference equations," Radovi Mathematićki, vol. 11, pp. 1-19, 2002.

Kulenović, M. R. S. and Nurkanović, M., "Asymptotic behavior of a linear fractional system of difference equations," J. Ineq. Appl., vol. 1, pp. 127-143, 2005 .

Leonard, W. J. and May, R., "Nonlinear aspects of competition between species," SIAM J. Appl. Math., vol. 29, pp. 243-275, 1975.

Leslie, P. H., "Some further notes on the use of matrices in population mathematics," Biometrika, vol. 55, pp. 213-245, 1948.

Leslie, P. and Gower, J., "The properties of a stochastic model for two competing species," Biometrika, vol. 45, pp. 316-330, 1958.

Li, J., Cali, L., and Li, Y., "Stage-structured wild and sterile mosquito population models and their dynamics," Journal of Biological Dynamics, vol. 11, pp. 79-101, 2017.

Livadiotis, G., Assas, L., Dennis, B., Elaydi, S., and Kwessi, E., "A discrete-time host-parasitoid model with an allee effect," Journal of Biological Dynamics, vol. 9, pp. 34-51, 2015.

May, R. M., "Host-parasitoid systems in patchy environments: A phenomenological model," Journal of Animal Ecology, vol. 17, pp. 833-844, 1978.

McCaig, C., Fenton, A., Graham, A., Shankland, C., and Norman, R., "Using process algebra to develop predator-prey models of within-host parasite dynamics," Journal of Theoretical Biology, vol. 329, pp. 74-81, 2013.

Nurkanović, M., "Asymptotic behavior of solutions to some two-dimensional systems of difference equations with applications," Ph.D. dissertation, University of Sarajevo, 2002.

Pardini, E., Drake, J., Chase, J., and Knight, T., "Complex population dynamics and control of the invasive biennial alliaria petiolata," Ecological Applications, vol. 19, pp. 387-397, 2009.

Park, T., "Experimental studies of inter-species competition: competition between populations of the flour beetles tribolium confusum duval and tribolium castaneum herbst," Ecological Monograph, vol. 18, pp. 265-308, 1948. 
Rebarber, R., Tenhumberg, B., and Townley, S., "Global asymptotic stability of density dependent integral projection models," Theoretical Population Biology, vol. 81, pp. 81-87, 2012.

Rees, M. and Rose, K., "Evolution of flowering strategies in oenothera glazioviana: an integral projection model approach," Proceedings of the Royal Society of London, vol. 269, pp. 1509-1515, 2002.

Smale, S., "On the differential equations of species in competition," Journal of Mathematical Biology, vol. 3, pp. 5-7, 1976.

Smith, H. L., "Periodic competitive differential equations and the discrete dynamics of competitive maps," Journal of Differential Equations, vol. 64, pp. 165-194, 1986.

Smith, H. L., "Periodic competitive differential equations and the discrete dynamics of competitive maps," Journal of Differential Equations, vol. 64, pp. 165-194, 1986.

Smith, H. L., "Planar competitive and cooperative difference equations," Journal of Difference Equations and Applications, vol. 3, pp. 335-357, 1998.

Smith, H. L. and Thieme, H. R., "Persistence and global stability for a class of discrete time structured population models," Discrete and Continuous Dynamical Systems, vol. 33, pp. 4627-4646, 2013.

Sternberg, S., Dynamical Systems. Dover Publications, 2013.

Tang, Y., "Global dynamics of a parasite-host model with nonlinear incidence rate," International Journal of Bifurcation and Chaos, vol. 25, pp. 1-9, 2015.

Townley, S., Rebarber, R., and Tenhumberg, B., "Feedback control systems analysis of density dependent population dynamics," Systems and Control Letters, vol. 61, pp. 309-315, 2012.

Ufuktepe, U. and Kapçak, S., "Stability analysis of a host-parasite model," $A d$ vances in Difference Equations, vol. 79, pp. 1-9, 2013.

Wang, T. and Zhang, Y., "Chemical control for host-parasitoid model within the parasitism season and its complex dynamics," Discrete Dynamics in Nature and Society, vol. 1, pp. 1-14, 2016.

Xiang, C. and Xiang, Z., "Discete switching host-parasitoid models with integrated pest control," International Journal of Bifurcation and Chaos, vol. 24, pp. 120, 2014.

Yang, J., Tang, S., and Tan, Y., "Complex dynamics and bifurcation analysis of host-parasitoid models with impulsive control strategy," Chaos, Solitons and Fractals, vol. 91, pp. 522-532, 2016. 